\title{
Lloyd Humberstone
}

\section{SUPERVENIENCE, DEPENDENCE, DISJUNCTION}

\begin{abstract}
This paper explores variations on and connections between the topics mentioned in its title, using as something of an anchor the discussion in Valentin Goranko and Antti Kuusisto's "Logics for propositional determinacy and independence", a venture into what the authors call the logic of determinacy, which they contrast with (a demodalized version of) Jouko Väänänen's modal dependence logic. As they make clear in their discussion, these logics are closely connected with the topics of noncontingency and supervenience. Two opening sections of the present paper address some of these connections, including related earlier logical work by the present author as well as very recent work by Jie Fan. The Väänänen-inspired treatment is presented in a third section, and then, in sections 4 and 5 , as a kind of centerpiece for the discussion, we follow Goranko and Kuusisto in elaborating one principal reason offered for preferring their own approach over that treatment, which concerns some anomalies over the behaviour of disjunction in the latter treatment. Sections 6 and 7 look at dependence and (several different versions of) disjunction in inquisitive logic, especially as presented by Ivano Ciardelli. Section 8 revisits the less formal propertysupervenience literature with issues from the first two sections of the paper in mind, and we conclude with a Postscript addressing a further conceptual issue pertaining to the relation between modal and quantificational dependence logics.
\end{abstract}

Keywords: Modal dependence logic; disjunction; supervenience, inquisitive logic; contingency; whether-disjunction; conservative extension

\section{Introduction and Background}

This paper, rather than defending any particular thesis, explores connections between, on the one hand, the philosophical and technical literature on supervenience among properties, and, on the other, some more recent 
literature on (modal) dependence logic. With such a broad terrain we promise something for everyone (with interests in this area) understood in the usual $\forall \exists$ sense rather than the $\exists \forall$ sense, so the remainder of this paragraph offers advice as to which sections will treat the topics of interest to which readers, for whom the cross-references supplied can be followed for any pre-requisites from elsewhere in the paper. A useful focus for such a comparative discussion is provided by Goranko and Kuusisto [2018], in which the authors propose a logic of dependence, or determinacy, as they put it, which they urge is better than the modal dependence logic of Väänänen [2008], in particular because of the treatment of disjunction in the latter logic. We get to that in sections $3-5$, and move on to the related area of inquisitive semantics in Section 6 and to another issue (the "or" in whether-constructions) it raises, in Section 7, before returning in Section 8 to some aspects of property supervenience - partly philosophical and partly formal - alluded to in the preceding sections, and a postscript tying up one conceptual loose end (Section 9).

Section 3 of Goranko and Kuusisto [2018] introduces what they label $\mathcal{L}_{\mathrm{D}}$ and refer to as the propositional logic of determinacy. Its language extends that of classical propositional logic by adding the operator $\mathrm{D}$ with formation rule: for any formulas $\varphi_{1}, \ldots, \varphi_{k}, \psi, \mathrm{D}\left(\varphi_{1}, \ldots, \varphi_{k} ; \psi\right)$ is a formula. This ('dependence') formula will be given a reading roughly along the following lines: the truth-values of $\varphi_{1}, \ldots, \varphi_{k}$ determine that of $\psi$, or again: the truth-value of $\psi$ depends at most on the truth-values of $\varphi_{1}, \ldots, \varphi_{k}$. This is a generalization of the corresponding formation rule for the language of the logic Goranko and Kuusisto call $\mathcal{D}$ in their earlier Section 2.6, and refer to there as the propositional logic of dependence; for its language the formation rule for $\mathrm{D}$-formulas is restricted to the case in which $\varphi_{1}, \ldots, \varphi_{k}, \psi$ are all propositional variables (sentence letters) rather than arbitrary formulas. Both the logics $\mathcal{L}_{\mathrm{D}}$ and $\mathcal{D}$ are characterized semantically, as are several others discussed in [Goranko and Kuusisto, 2018], though not here. We treat $\mathcal{L}_{\mathrm{D}}$ in the present section, returning to $\mathcal{D}$ in the next. ${ }^{1}$ The latter goes back to Väänänen [2008], as a modal variation on the dependence and independence logics of Väänänen [2007a], themselves inspired by the game-theoretic seman-

1 Two other logics, focussed on a notion of independence rather than dependence, $\mathcal{I}$ and $\mathcal{L}_{1}$, in something of the same spirit as $\mathcal{D}$ and $\mathcal{L}_{\mathrm{D}}$, respectively, are also treated in [Goranko and Kuusisto, 2018] but will not be discussed here. 
tics for Henkin (or 'branching') quantifiers of Hintikka and co-authors, later re-packaged and marketed as independence-friendly (or ' $I F$ ') logic, and by the provision for the latter of a compositional model-theoretic semantics in [Hodges, 1997]. ${ }^{2}$ None of these historical roots will concern us here; for more information on them, see [Goranko and Kuusisto, 2018] and the references there supplied, as well as [Tulenheimo, 2016]. (In a final Postscript section we return to quantificational dependence logic for contrastive purposes.) Nor, as will be gathered from the above informal glosses on the newly available compounds, will any terminological contrast be observed between talk of determination/determinacy and talk of dependence. (See further Remark 1.1 below.)

There is a standard possible worlds semantics, in the style of Kripke or more accurately, of Carnap, given for the propositional logic of determinacy in [Goranko and Kuusisto, 2018], using models $\mathcal{M}=\langle W, V\rangle-$ at least as I shall expound matters here, moving from a Carnapian to a Kripkean approach - with $W$ a non-empty set, subsets of which are assigned to propositional variables $p_{i}(i=1, \ldots, n, \ldots)^{3}$ by the function $V$, and truth defined for formulas of $\mathcal{L}_{\mathrm{D}}$ inductively thus, with " $\mathcal{M}, w \models \varphi$ " for " $\varphi$ is true at $w$ in $\mathcal{M}$ "; here $\mathcal{M}$ is as above and $w \in W$ :

- $\mathcal{M}, w \models p_{i}$ iff $w \in V\left(p_{i}\right)$

- $\mathcal{M}, w \models \neg \varphi$ iff $\mathcal{M}, w \not \models \varphi$

- $\mathcal{M}, w \models \varphi \rightarrow \psi$ iff $\mathcal{M}, w \not \models \varphi$ or $\mathcal{M}, w \models \psi$

- $\mathcal{M}, w \models \mathrm{D}\left(\varphi_{1}, \ldots, \varphi_{k} ; \psi\right)$ iff for all $u, v \in W$, if for all $i \leq k$ we have $\mathcal{M}, u \models \varphi_{i} \Leftrightarrow \mathcal{M}, v \models \varphi_{i}$, then we have $\mathcal{M}, u \models \psi \Leftrightarrow \mathcal{M}, v \models \psi$.

Although the substance of these four conditions in the definition of truth a point, and the selection of Boolean connectives treated, is that of Goranko and Kuusisto [2018, §3], the notation is not quite theirs. [Goranko and Kuusisto, 2018] simply relativizes truth to the pair consisting of $W$ and some element $w$ thereof, taking $W$ to be a set, specifically, of truth-value assignments to the propositional variables, and they

2 See also [Hodges, 2007]. An earlier gesture in the direction of a compositional treatment, though this time in the 'homophonic truth-theory' tradition, can be found in [Humberstone, 1987]; Patton [1989] (perhaps justifiably) provides an unfavourable assessment of its prospects. Writing in 1981, Stephen Read [1981, p. 41] remarks: "[G]ame theory is indeed psychologically illuminating, and has prompted certain insights - e.g. in urn models and branching quantifiers - yet any of these can be accommodated in a recursive approach." It is not obvious exactly what he had in mind for the branching quantifier case.

${ }^{3}$ In general we write $p_{1}, p_{2}, p_{3}, p_{4}$ as $p, q, r, s$. 
call such sets themselves models - or sometimes $S D$-models, the letters abbreviating 'State Description(s)'. (Because their elements are assignments, they have no need for $V$ to play this role, and no option for distinct elements of $W$ to make precisely the same such assignment. In addition, Goranko and Kuusisto allow $W$ to be empty.) The changes made here in the presentation are in line with the following remark (just before Definition 3.1) in [Goranko and Kuusisto, 2018], though going a bit further in the direction suggested: "The semantics for $\mathcal{L}_{\mathrm{D}}$ is similar to Kripke semantics in the case where the accessibility relation of a model $W$ is the universal relation $W \times W$." The authors go on to say that a valid formula is most naturally defined, with this analogy in mind, as one true on each assignment in any $W$, or, putting this in terms of the above reformulation, true at every point in every model. We might go further and define the induced 'local' consequence relation for which we will use the same " $=$ " notation as for the truth-relation above:

(D1.1) $\varphi_{1}, \ldots, \varphi_{n} \models \psi$ iff for every model $\mathcal{M}=\langle W, V\rangle$ and all $w \in W$, if $\mathcal{M}, w \models \varphi_{i}$ for each $i \leq n$, then $\mathcal{M}, w \models \psi$.

One can think of the connective D as rendering within the object language the metalinguistic concept of a consequence relation's being supervenience determined by a class of valuations, where the latter are conceived as assignments of truth-values $(T$ and $F$ ) to arbitrary formulas of the language - rather than just the propositional variables. (Valuations will always be total bivalent valuations in what follows.) Where $\mathcal{V}$ is a collection of such valuations for a language the consequence relations $\vdash_{\mathcal{V}}^{\text {inf }}$ and $\vdash_{\mathcal{V}}^{\text {svc }}$, respectively inference-determined and superveniencedetermined by $\mathcal{V}$, are defined by:

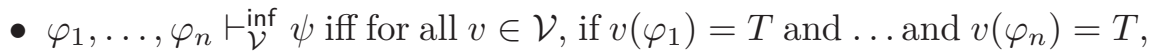
then $v(\psi)=T$.

- $\varphi_{1}, \ldots, \varphi_{n} \vdash_{\mathcal{V}}^{\text {svc }} \psi$ iff for all $u, v \in \mathcal{V}$, if $u\left(\varphi_{1}\right)=v\left(\varphi_{1}\right)$ and $\ldots$ and $u\left(\varphi_{n}\right)=v\left(\varphi_{n}\right)$, then $u(\psi)=v(\psi)$.

Of course it is the first of these that is usually just called the consequence relation determined by $\mathcal{V}$, since we are typically interested in preservation of truth on arbitrary valuations in the chosen $\mathcal{V}$ rather than preservation of agreement in truth-value for arbitrary pairs of valuations from $\mathcal{V}$. Readers already familiar with the 'supervenience' terminology should skip the following remark. 
Remark 1.1. We use the term "supervenience" because of its use by philosophers in discussing properties: a property is said to be supervenient on a set of properties when it is impossible for two individuals to differ in respect of the given property if they are alike in respect of all properties in the set. Several precisifications of this idea of (as we shall call it) property supervenience, as well as variations on it, can be found in the philosophical literature; good surveys are provided by McLaughlin [1995], McLaughlin and Bennett [2014], and Steinberg [2013]; see also the discussion and references in Section 8 below, where issues touched on here or related to themes in this section and the next on are taken up. (By considering, instead, pairs of $n$-tuples of individuals, we get the corresponding notion of supervenience among $n$-ary relations, which will not be of special interest here [see Leuenberger, 2013].) It should be added that talk of dependence and talk of supervenience - interchangeable for present purposes - may have rather different connotations in a philosophical setting, where talk of one property's dependence on others can suggest that something's having the former is grounded in, due to, based on, or arises in virtue of its having the latter. The confusion of this relation with supervenience has been emphasized in [Dancy, 2004, e.g., p. 87], where dependence in this stronger sense is called - adapting a usage of W. D. Ross - resultance; see also the earlier [Dancy, 1981]. (Similar concerns are aired in [Zangwill, 2008] and [DePaul, 1987]; oppositions between supervenience and dependence can be found in many further places [Moser and Trout, 1995, esp. §3].) The same applies to somewhat similarly intended talk of determination: see $\S 4$ of [Petrie, 1987], and [Hellman, 1985]; Yoshimi [2007] has his own dependence/determination contrast. In part to avoid these extraneous connotations, Kim Kim [1990] has suggested that supervenience simpliciter might be less confusingly called 'covariance', though this terminology misleadingly suggests that the relation of supervenience - conceived as a relation between classes of properties - is symmetric, which is not the case (pace Miller [1990]: see [Hellman, 1992] or [Heil, 1995, p. 162], for a diagnosis of the mistake in [Miller, 1990]); Heil [1995] similarly disposes of a claim by Miller - cf. also [Sonderholm, 2007] - that most widely touted supervenience claims are trivial because agreement in respect of the underlying properties suffices for the identity of the individuals concerned. Similarly, McFetridge [1985, p. 257] speaks of 'principles of (conditional) coincidence' though the parenthetical insertion is my addition to forestall an unintended symmetric interpretation again. (Yoshimi [2007, p. 119] says that the rela- 
tions of dependence and determination under discussion there are asymmetric, but this seems to be a slip - at least when applied to these defined up to that point - for non-symmetric.) The present discussion does not venture into the analogue of the stronger resultance-style kinds of dependence, often going under the name of grounding, which have raised interesting questions [Wilson, 2018] and objections ([Wilson, 2014] - an unrelated Wilson) of their own. Some steps have been taken in this direction for propositional logic, however, for instance in [Schnieder, 2008]; see also [Correia, 2010] and references therein, as well as several papers in the collection [Hoeltje et al., 2013]. While the perceived philosophical significance of the purely covariational supervenience notions (to use a plural form anticipating the 'Second Comment' in Section 8 below) has declined as their difference from the grounding-like relations has gradually sunk in, there remains much of logical interest to extracted from them, as the present paper hopes to show. Compounding the risks of confusion in this area is the distinction between dependence as dependence on what suffices for settling something and dependence as dependence on what is needed for settling something, raised below in note 17. In the present case the individuals are the valuations in $\mathcal{V}$ and the given property is that of verifying $\psi$ while the properties on the set of which its supervenience is at issue are those of verifying $\varphi_{1}$, of verifying $\varphi_{2}, \ldots$, of verifying $\varphi_{n}$.

An equivalent characterization of $\vdash_{\mathcal{V}}^{\text {svc }}$, favoured in [Humberstone, 2011, p. 1142], which could be used in place of the definition given above, is provided by:

Proposition 1.2. For arbitrary formulas $\varphi_{1}, \ldots, \varphi_{n}, \psi$, we have $\varphi_{1}, \ldots$, $\varphi_{n} \vdash \mathcal{V}$ svc $\psi$ if and only if there is a function $f:\{T, F\}^{n} \longrightarrow\{T, F\}$ such that for all $v \in \mathcal{V}$,

$$
v(\psi)=f\left(v\left(\varphi_{i}\right), \ldots, v\left(\varphi_{n}\right)\right) .
$$

As in Humberstone [1996a] and elsewhere, we define the binary operation $\sim$ of equivalential combination on valuations by:

$$
u \sim v(\varphi)=T \text { if and only if } u(\varphi)=v(\varphi)
$$

for all formulas $\varphi$. Lifting this in the usual power-algebraic way to an operation on pairs of sets of valuations $\mathcal{U}$ and $\mathcal{V}$ by putting

$$
\mathcal{U} \sim \mathcal{V}=\{u \sim v \mid u \in \mathcal{U}, v \in \mathcal{V}\}
$$

we have, for any $\mathcal{V}: \vdash_{\mathcal{V}}^{\text {svc }}=\vdash_{\mathcal{V}}^{\text {inf }} \sim \mathcal{V}$. 
The relations $\vdash_{\mathcal{V}}^{\text {svc }}$ are not without interest. For example, they are useful in fleshing out the observation ${ }^{4}$ that database functional dependency relations are consequence relations (see [Humberstone, 1993]), and also in yielding a simple a proof of Surma's Deduction Theorem, given as (1.1) below, for the equivalential fragment of classical logic-i.e., for the restriction, $\vdash$, of the classical consequence relation to the language whose only connective is $\leftrightarrow$ - according to which

$$
\varphi_{1}, \ldots, \varphi_{n} \vdash \psi \text { iff } \varphi_{1}, \ldots, \varphi_{n-1} \vdash \varphi_{n} \leftrightarrow \psi \text { or } \varphi_{1}, \ldots, \varphi_{n-1} \vdash \psi \text {. }
$$

Full details can be found in [Humberstone, 2011, p. 1132] (Observation 7.31.2 and Exercise 7.31.3(iii) $)^{5}$ ), but the basic idea can be explained here. The consequence relation mentioned in (1.1) is that inferencedetermined by the class of all valuations $v$ which are $\leftrightarrow$-Boolean in the sense that for all formulas $\varphi, \psi, v(\varphi \leftrightarrow \psi)=T$ iff $v(\varphi)=v(\psi)$ and, taking the class of all these valuations as $\mathcal{V}$ we have the special feature that for this choice of $\mathcal{V}, \mathcal{V}=\mathcal{V} \sim \mathcal{V}$, so this particular $\vdash$ is both inference-determined and supervenience-determined by the class of $\leftrightarrow$ Boolean valuations. For the claim that $\mathcal{V}$ is closed under the operation $\sim$ (i.e., $\mathcal{V} \sim \mathcal{V} \subseteq \mathcal{V}$ ) is actually equivalent to the claim that $\mathcal{V}=\mathcal{V} \sim \mathcal{V}$ [see Humberstone, 1993, p. 321]. And any consequence relation $\vdash$ inferencedetermined by a class of valuations closed under $\sim$ is easily seen to satisfy the following condition, for all sets $\Gamma$ of formulas, and all formulas $\varphi, \psi$ :

$$
\text { If } \Gamma, \varphi \vdash \psi \text { then } \Gamma \vdash \psi \text { or } \Gamma, \psi \vdash \varphi \text {. }
$$

(1.1) is an easy consequence of this 'Exchange Property', as (1.2) is called in [Humberstone, 1996a] (where its appearance in other mathematical areas is also indicated: see $\S 0.4$, as well as $§ 0.6$ and in subsequent sections of [Humberstone, 1996a]).

Remarks 1.3. (i) Generalized (or multiple-conclusion) consequence relations - for present purposes denoted by (possibly further decorated) "I-" - are well known in the literature, and the present contrast could naturally be extended to cover them. Thus for a class $\mathcal{V}$ of valuations we have the generalized consequence relation $\Vdash \Vdash_{\mathcal{V}}^{\text {inf }}$ defined by:

4 Familiar from, e.g., [Fagin, 1977; Rauszer, 1985; Sagiv et al., 1981], though not put in exactly these terms; a fuller listing of sources can be found in - and just before - the bibliography of [Humberstone, 1993].

5 This exercise addresses explicitly the 'only if' half of the inset claim, the 'if' half being obvious. 


$$
\begin{aligned}
\varphi_{1}, \ldots, \varphi_{m} \Vdash_{\mathcal{V}}^{\text {inf }} \psi_{1}, \ldots, \psi_{n} \text { iff } \quad & \text { for all } v \in \mathcal{V} \text {, if } v\left(\varphi_{i}\right)=T \text { for } \\
& \text { each } i(1 \leq i \leq m), \text { then } \\
& v\left(\psi_{j}\right)=T \text { for some } j(1 \leq j \leq n) .
\end{aligned}
$$

In the same vein one could consider a generalized version of supervenience determination:

$$
\begin{aligned}
\varphi_{1}, \ldots, \varphi_{m} \Vdash_{\mathcal{V}}^{\text {svc }} \psi_{1}, \ldots, \psi_{n} \text { iff } \text { for all } u, v \in \mathcal{V}, \text { if } u\left(\varphi_{i}\right)=v\left(\varphi_{i}\right) \text { for } \\
\text { each } i \text {, then } u\left(\psi_{j}\right)=v\left(\psi_{j}\right) \text { for some } j .
\end{aligned}
$$

(Note that $\S 3$ of [Humberstone, 1992] uses "|ト" for " $\vdash$ svc" rather than for generalized consequence relations. Section 3 below makes yet another unrelated use, taken over from [Goranko and Kuusisto, 2018], of this same notation for a special verification/satisfaction notion, further adapted in (D3.1) on p. 37 to refer to an associated consequence relation. Since we are not there concerned with generalized consequence relations, no confusion will arise.) For an illustration with $m=0, n=3$, and $\mathcal{V}$ as the class of all $\leftrightarrow$-Boolean valuations, we have for all $\varphi, \psi$ : $\Vdash_{\mathcal{V}}^{\text {svc }} \varphi, \psi, \varphi \leftrightarrow \psi$. This is not a very surprising example given the above discussion of closure under $\sim$. Further illustration of the issues involved with supervenience-determined generalized consequence relations can be found in Proposition 1.4 and Example 2.2 below.

(ii) Note that in the definition of $\Vdash_{\mathcal{V}}^{\text {svc }}$ just given, we have "for some $j$ " rather than "for all $j$ " to handle the $n \neq 1$ case on the right, essentially following Gentzen's example. (With "all" the result would not be a generalized consequence relation since we would not satisfy the monotonicity - or 'weakening' - condition $\Gamma \subseteq \Gamma^{+}, \Delta \subseteq \Delta^{+}$and $\Gamma \Vdash \Delta$ imply $\Gamma \Vdash^{+} \Delta$. With "some" on the right we retain the identity of $\Vdash_{\mathcal{V}}^{\text {svc }}$, with $\Vdash \Vdash_{\mathcal{V}}^{\text {inf }} \sim \mathcal{V}$, the latter understood as the generalized consequence relation determined by $\mathcal{V} \sim \mathcal{V}$ in the usual sense.) This is worth noting because Galliani and Väänänen [2014, p. 104] who take as new atomic formulas $=(\vec{y}, \vec{x})$ where $\vec{y}, \vec{x}$ are sequences of individual variables and the informal reading offered is "the $\vec{y}$ totally determine the $\vec{x}$ ". The analogue of this in the present case with our definition of " $\Vdash_{\mathcal{V}}^{\text {svc }}$ ", though let us suppress the super- and subscripts for readability, when sequences of variables are replaced by sets of formulas, would not be " $\Gamma \Vdash \Delta$ " but rather " $\Gamma \Vdash \psi$ for each $\psi \in \Delta$." (We resume this theme in the discussion of $\forall$-reducibility in Section 8.) This kind of abbreviative notation is indispensable for practical purposes in several areas - for example in many of the formulations used in algebraic logic (cf. the definitions of what it is to be a set of equivalence formulas or a set of implication formulas given on p. 47 of 
[Font et al., 2006] or conditions (4) and (5) at the top of p. 48 there) and no harm is done as long as the two uses of 'multiplicity on the right' are clearly distinguished. In [Fan, 2016] he extends his $\left(\varphi_{1}, \ldots, \varphi_{n}\right) \Rightarrow \psi$ notation to allow also $\left(\varphi_{1}, \ldots, \varphi_{n}\right) \Rightarrow\left(\psi_{1}, \ldots, \psi_{m}\right)$ in the abbreviative way, thereby following [Galliani and Väänänen, 2014] rather than using this for the connective analogous to the relation $\Vdash_{\mathcal{V}}^{\text {svc }}$ as understood here.

(iii) In [Humberstone, 1993] a class $\mathcal{V}$ of valuations was said to be consilient with a consequence relations $\vdash$ just in case for there is no set of formulas $\Gamma \cup\{\psi\}$ for which we have $\Gamma \vdash \psi$ and $u, v \in \mathcal{V}$ with $u, v$ agreeing on all $\varphi \in \Gamma$ yet not on $\psi$. This notion naturally lifts to the case of generalized consequence relations by defining $\mathcal{V}$ to be consilient with $\Vdash$ when for no $\Gamma, \Delta$ can there be found $u, v \in \mathcal{V}$ agreeing on all formulas in $\Gamma$ while differing on all formulas in $\Delta$. But a word of warning should have been sounded on the danger of thinking that this is completely parallel to talk of a class of valuations $\mathcal{V}$ being consistent with $\vdash$ (or $\Vdash$ ) - a parallel the terminology was deliberately chosen to suggest. The latter means that no valuation in the class verifies the formulas on the left but not any formula(s) on the right of the any correct $\vdash / \Vdash$-statement. And the danger is that the latter is a property $\mathcal{V}$ has in virtue of each of its elements being consistent with $\vdash$ or $\Vdash$ in the sense of verifying (all of) the left-hand formulas in any correct $\vdash / \Vdash$-statement, the but not (any of) the right-hand formulas. There is no corresponding notion of an individual valuation's being consilient with a consequence or generalized consequence relation - the analogue would be of a pair $\langle u, v\rangle$ 's being consilient with such a relation. Thus, for example, by contrast with the consistency case, there is no such thing as the set of all valuations consilient with a given (generalized) consequence relation.

(iv) One could also investigate the relation holding between two sets, $\Gamma$ and $\Delta$ of premisses and a single conclusion, with the conclusion settled superveniencewise on the basis of $\Delta$, with verifying all formulas in $\Gamma$ supplying a background condition on the valuations considered. Writing $\Gamma ; \Delta \vdash_{\mathcal{V}} \psi$ for this relation, it would be defined by:

$\Gamma ; \Delta \vdash_{\mathcal{V}} \psi$ iff for all $u, v \in \mathcal{V}$ if $u(\chi)=v(\chi)=T$ for all $\chi \in \Gamma$ then if for all $\varphi \in \Delta, u(\varphi)=v(\varphi)$, we must have $u(\psi)=v(\psi)$.

This would be (one kind of) hybrid between inference determined and supervenience determined consequence. (This remark was suggested by the discussion of 'conditional dependencies' of [Ciardelli, 2016b, p. 140].) $\triangleleft$ 
We illustrate the ideas of Remark 1.3(i) for a case in which because the inference-determined and supervenience-determined consequence relations coincide, so that we can state the relevant observations without the superscript "svc". Let $\mathcal{V}_{\leftrightarrow}$ be the class of $\leftrightarrow$-Boolean valuations for a language with $\leftrightarrow$ among its primitive connectives - for definiteness we take this to be the sole connective and call the formulas in this fragment $\leftrightarrow$-formulas - and $\vdash_{\leftrightarrow}$ be the generalized consequence relation inference-determined $\mathcal{V}_{\leftrightarrow}$. Here, as already remarked, $\Vdash_{\leftrightarrow}^{\leftrightarrow}=\Vdash_{\mathcal{V}_{\leftrightarrow}}^{\text {inf }}=\Vdash_{\mathcal{V}}^{\text {svc }}$, since $\mathcal{V}_{\leftrightarrow}=\mathcal{V}_{\leftrightarrow} \sim \mathcal{V}_{\leftrightarrow}$. Proposition 1.4(i), is a generalization of (1.2), proved in the same way, replacing the formula variable " $\psi$ " with the set variable " $\Delta$ "; a similar replacement cannot be made for " $\varphi$ ". Proposition 1.4(ii) gives what in the presence of an explicit disjunction connective (w.r.t. which our generalized consequence relation was $\vee$-classical) might be called the Binary Disjunction Property (with side-formulas on the left) for classical equivalential logic; note the "Binary": even with no such side-formulas ( $\Gamma=\varnothing$, that is), we do not have the corresponding property for the case of three formulas on the right, since for example $\Vdash_{\leftrightarrow} p, q, p \leftrightarrow q$ without $\Vdash_{\leftrightarrow} p, \Vdash_{\leftrightarrow} q$, or $\Vdash_{\leftrightarrow} p \leftrightarrow q$.

Proposition 1.4. (i) For any set $\Gamma \cup \Delta \cup\{\varphi\}$ of $\leftrightarrow$-formulas, if $\Gamma, \varphi \Vdash_{\leftrightarrow} \Delta$ then either $\Gamma \Vdash_{\leftrightarrow} \Delta$ or $\Gamma, \Delta \Vdash_{\leftrightarrow} \varphi$.

(ii) For any set $\Gamma \cup\{\varphi, \psi\}$ of formulas of $\leftrightarrow$-formulas, if $\Gamma \Vdash_{\leftrightarrow} \varphi, \psi$ then either $\Gamma \Vdash_{\leftrightarrow} \varphi$ or $\Gamma \Vdash_{\leftrightarrow} \psi$.

Proof. For (ii): Given a valuation $v$, we write " $v(\Gamma)=T$ " for " $v(\chi)=$ $T$ for all $\chi \in \Gamma$ ". For a contradiction, suppose that (1) $\Gamma \Vdash_{\leftrightarrow} \varphi, \psi$ but (2) $\Gamma \nVdash_{\leftrightarrow} \varphi$ and (3) $\Gamma \nVdash_{\leftrightarrow} \psi$. By (2) there exists $u \in V_{\leftrightarrow}$ with $u(\Gamma)=T, u(\varphi)=F$, and, appealing to $(1), u(\psi)=T$. Similarly, by $(3)$ $v \in V_{\leftrightarrow}$ with $v(\Gamma)=T, v(\psi)=F$, and, because of (1) again, $v(\varphi)=T$. From these assignments we see that $u \sim v(\Gamma)=T, u \sim v(\varphi)=F$, and $u \sim v(\psi)=F$, but since $V_{\leftrightarrow}$ is closed under $\sim$, this contradicts (1).

It would be good to have something of a generality comparable to (1.2) to report as condition satisfied by all supervenience-determined consequence relations (i.e., those inference-determined by some $\mathcal{V} \sim \mathcal{V}$ ), preferably for a condition necessary and sufficient for being supervenience-determined, but [Humberstone, 1993] leaves this as an open problem, after mentioning a failed conjecture concerning such a condition 
(namely $(* * *)$ on p. 332 of [Humberstone, 1993]). ${ }^{6}$ Some special cases are mentioned and we repeat them here without supporting argument (if necessary, see the proofs of propositions 3.6 and 3.8 in [Humberstone, 1993]), the first just being the case of the condition above with $\Gamma=\varnothing$ :

EXAMPLES 1.5. (i) If $\vdash=\vdash \mathcal{V}$ svc for some $\mathcal{V}$, then $\vdash$ satisfies for all formulas $\varphi, \psi$ in the language of $\vdash$ :

$$
\text { If } \varphi \vdash \psi \text { then either } \vdash \psi \text { or } \psi \vdash \varphi \text {. }
$$

(ii) For $\vdash$ as in (i), we have for all formulas $\varphi, \psi, \chi$ :

$$
\text { If } \varphi, \psi \vdash \chi \text { and } \varphi, \chi \vdash \psi \text { then } \varphi \vdash \chi \text { or } \psi, \chi \vdash \varphi \text {. }
$$

(iii) With the semicolon notation for inference/supervenience hybrid relations $\vdash$ mentioned in Remark 1.3(iv), we have a suitably repunctuated incarnation of (1.2):

$$
\text { If } \Gamma ; \varphi \vdash \psi \text { then } \Gamma \vdash \psi \text { or } \Gamma ; \psi \vdash \varphi \text {. }
$$

We return to some of these conditions in Section 2.

Remark 1.6. In view of (especially the final sentence of) Remark 1.1, we can also construe Examples 1.5 as telling us something about property supervenience. For instance, in this setting, Example 1.5(i) tells us that when supervenience is thought of as a binary relation between properties, this is relation is 'almost symmetric': if $P$ is supervenient on $Q$, then $Q$ is supervenient on $P$ except in the case that $P$ is possessor-independent, in the sense that everything or nothing has property $P$. (For present purposes we identify a property with a set of individuals; more refined approaches will be considered under 'Second Comment' in Section 8.) Note that the denial of supervenience included in Remark 1.1 concerned not this relation but supervenience as a relation between classes of properties, in terms of which the current relation would be the special case " $\{P\}$ is supervenient on $\{Q\}$ ". In the Intermission in Section 8 it will be argued that neither of these should be taken as the fundamental supervenience relation, which instead should be taken as defined at the

${ }^{6}$ It also appears as Problem 1.1 .1 on p. 35 of [Humberstone, 1996a]; pp. 36 and 37 make some inconclusive suggestions toward solving the problem. Replacing consequence relations with preorders - i.e., considering the consequences only of unit sets - Theorem 4.6 of [Humberstone, 1992] gives a characterization of those preorders which are supervenience-determined. The condition numbered (38) on p. 125 there purports to be a correct partial generalization of Examples 1.5, but in view of the fate of $(* * *)$ in [Humberstone, 1993], its correctness may well be in doubt. 
start of Remark 1.1: as a relation between a single property and a class of properties - though this is still very vague and open to clarification in non-equivalent ways (as indicated in Section 8).

Reasoning similar to that underlying Examples 1.5 allows us parallel conclusions for Goranko and Kuusisto's $\mathcal{L}_{\mathrm{D}}$, as we illustrate for simplicity with Example 1.5(i). For the consequence relation $\models$ defined in (D1.1) on p. 6, we have, for all formulas $\varphi, \psi$ :

$$
\mathrm{D}(\varphi ; \psi) \models \mathrm{D} \psi \vee \mathrm{D}(\psi ; \varphi) .
$$

Naturally, if one preferred to put matters in terms of the outright validity of formulas, the current point would be a matter of the validity of any formula of the form $\mathrm{D}(\varphi ; \psi) \rightarrow(\mathrm{D} \psi \vee \mathrm{D}(\psi ; \varphi))$.

The idea of taking supervenience into the object language, as is done here with D, also occurred independently to Jie Fan [2016], whose study of the connective concerned is in one respect less general and in one respect more general than that of Goranko and Kuusisto. The respect in which it is less general is that it considers mainly the $n=1$ (and in passing the $n=0)$ case of $\mathrm{D}\left(\varphi_{1}, \ldots, \varphi_{n} ; \psi\right)$, though certainly the general case is given some consideration under the heading 'generalized supervenience' in a later section of [Fan, 2016]. The respect in which it is more general is that it does not restrict attention to the models whose accessibility relation is the universal relation. ${ }^{7}$ As a step towards explaining the greater generality pursued in this respect, we can consider first an intermediate formulation, using a binary accessibility relation $R$ so that models $\mathcal{M}$ are taken to have the familiar form $\langle W, R, V\rangle$, and we write $R(w)$ for $\{x \in W \mid R w x\}$, altering only the clause in the definition of $\models$ from the second paragraph above by replacing "for all $u, v \in W$ " with "for all $u, v \in R(w)$ ". Thus now D-formulas may take different truth-values at different points in the same model, since-taking the one-place case - for $\psi$ to be supervenient on, or depend on (at most) $\varphi$ at $w$ is for there to be no two points accessible to $w$ at which $\varphi$ has the same truth-value while $\psi$ has different truth-values. As Fan observes, this $n=1$ case, in which $\mathrm{D}$ (not that this is the notation used in [Fan, 2016]) becomes a binary connective, analogous to strict implication in conventional modal logic, except that whereas strict implication internalizes in the object language the one-premiss version of consequence by

7 This restriction is also removed in [Ciardelli, 2016a], as remarked on p. 238 there. 
inference, this new binary connective internalizes the one-premiss version of consequence by supervenience. Sticking to this $n=1$ case, we can now pass, for the record, to the actual semantics used by Fan, which instead equips models with a ternary accessibility relation $S$ which has a special case the relation holding between $w, x$ and $y$ (in that order) when on the intermediately general form just considered, $R w x$ and $R w y$, as in [Humberstone, 2002]; for the general case we subscript the first term, writing $S_{w} x y$ rather than $S w x y$. In this most general version of the semantics we have instead, parallelling Goranko and Kuusisto's formulation for comparative purposes:

- $\mathcal{M}, w \models \mathrm{D}(\varphi ; \psi)$ iff for all $u, v$ such that $S_{w} u v$, if $\mathcal{M}, u \models \varphi \Leftrightarrow$ $\mathcal{M}, v \models \varphi$, then $\mathcal{M}, u \models \psi \Leftrightarrow \mathcal{M}, v \models \psi$.

Using an argument involving suitably defined bisimulations, Fan observes is that this binary connective - and Fan writes the compounds concerned as $\varphi \Rightarrow \psi$ rather than as $\mathrm{D}(\varphi ; \psi)$ - cannot be defined in terms of the $n=0$ version (together with the Boolean connectives) written as $O$ in [Humberstone, 2002]:

- $\mathcal{M}, w \models \mathrm{D} \psi$ iff for all $u, v$ such that $S_{w} u v, \mathcal{M}, u \models \psi \Leftrightarrow \mathcal{M}, v \models \psi$.

For a more succinct formulation below, we introduce the following abbreviation (as in [Humberstone, 1993]):

(D1.2) We write $u \equiv_{\psi}^{\mathcal{M}} v$ for “ $\mathcal{M}, u \models \psi \Leftrightarrow \mathcal{M}, v \models \psi$ ".

Thus a generalization of the Goranko-Kuusisto apparatus in the style of [Fan, 2016] would take the following form:

- $\mathcal{M}, w \models \mathrm{D}\left(\varphi_{1}, \ldots, \varphi_{n} ; \psi\right)$ iff for all $u, v$ such that $S_{w} u v$, if $u \equiv_{\varphi_{i}}^{\mathcal{M}} v$ for all $i(i=1, \ldots, n)$, then $u \equiv_{\psi}^{\mathcal{M}} v$.

Note that in the special case in which " $S_{w} u v$ " amounts to " $R w u$ and $R w v$ " with $R$ thought of as relative possibility, $\mathrm{D} \psi$ says that it is noncontingent whether $\psi$. A common notation for this is $\Delta \psi$ (with $\nabla \psi$ for "it is contingent whether $\varphi$ "). Instead of $\mathrm{D} \psi$ for the $n=0$ case, Goranko and Kuusisto [2018] write C $\psi$ - thinking of " $C$ " as suggesting Constant though this is potentially confusing because in this case we are precisely

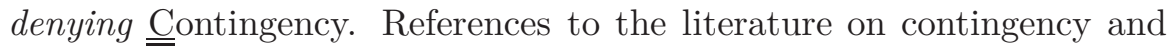
noncontingency based modal logics (taking $\Delta$ or $\nabla$ as the non-Boolean primitive rather than $\square$ or $\diamond$ ) can be found in [Goranko and Kuusisto, 2018] and in note 11; we touch on this topic again in Section 5. 
The Goranko-Kuusisto connective D is a multigrade connective in the sense that it forms compounds from any number of formulas $\varphi_{i}$ together with one formula $\psi$. If one restricts attention to connectives of fixed arity, this single connective $\mathrm{D}$ dissolves into countably many, one $-\mathrm{D}_{n}$, say - for each $n$, where, given formulas $\varphi_{1}, \ldots, \varphi_{n}$ and $\psi$, we have the formula $\mathrm{D}_{n}\left(\varphi_{1}, \ldots, \varphi_{n} ; \psi\right){ }^{8}$ Then Fan's observation, reported above, would be that $D_{1}$ - his binary supervenience connective $\Rightarrow$ - cannot be defined in terms of the Boolean connectives and $D_{0}$.

And just as $D_{1}$ can't be defined in terms of the $D_{0}$, so the ternary version, $D_{2}$ can't be defined in terms of the binary $D_{1}$. More generally, $\mathrm{D}_{n}\left(\varphi_{1}, \ldots, \varphi_{n} ; \psi\right) w$ in one of our models just in case:

For all $x, y$ if $S_{w} x y$ then if $x \equiv_{\varphi_{1}}^{\mathcal{M}} y$ and $\ldots$ and $x \equiv_{\varphi_{n}}^{\mathcal{M}} y$, then $x \equiv_{\psi}^{\mathcal{M}} y$.

We can define $\mathrm{D}_{n}$ in terms of $\mathrm{D}_{n+1}$ easily enough, for example defining the binary connective $D_{1}$ in terms of the ternary $D_{2}$ by putting $\mathrm{D}(\varphi ; \psi)=\mathrm{D}_{2}(\top, \varphi ; \psi)$. (We could equally well use $\perp$ here, of course.) But a definition in the reverse direction is not possible. For such a definition, we'd need, for any formulas $\varphi_{1}, \varphi_{2}$, a formula $\chi$ with property that agreement between any points $x, y$ in respect of $\chi$ was equivalent to agreement between those point in respect of $\varphi_{1}$ and in respect of $\varphi_{2}$. Another way of saying the latter is that we want $\equiv_{\chi}^{\mathcal{M}}$ to be the intersection of $\equiv_{\varphi_{1}}^{\mathcal{M}}$ and $\equiv_{\varphi_{2}}^{\mathcal{M}}$. But (as remarked in [Humberstone, 1992, p. 123]) this can't be arranged in general because the equivalence relations $\equiv_{\varphi}^{\mathcal{M}}$ are always bipartite in the sense that for any $x, y, z$ we have:

$$
x \equiv_{\varphi}^{\mathcal{M}} y \text { or } y \equiv_{\varphi}^{\mathcal{M}} z \text { or } x \equiv_{\varphi}^{\mathcal{M}} z,
$$

that is, there are only two equivalence classes for each such relation, ${ }^{9}$ whereas if we take the intersection of a $k$-partite equivalence relation and an $\ell$-partite such relation, in the worst case, we will be looking at

8 One might say that a unigrade treatment of $D$ is possible, even if this makes it no longer a straightforward sentence connective: it takes a set of formulas and a formula to make a formula. In the present case this would work, though in general multigrade connective can't be treated in exactly this way - for example multigrade exclusive disjunction [see McCawley, 1993, p. 287f.; or Humberstone, 2011, p. 783]. In this particular case replacing sets with multisets would do, however.

9 In terms of partitions: only two blocks, where "only" means "at most" - on the standard account of partitions. If we want to have exactly two blocks in all cases, we need to allow $\{\varnothing, W\}$ to count as a partition of $W$, i.e., to work with what are called Ore-partitions in [Humberstone, 2016]. 
a $(k \times \ell)$-partite relation. So for arbitrary $\varphi_{1}, \varphi_{2}$ and $\mathcal{M}$ there will in general be no $\chi$ for which $\equiv_{\chi}^{\mathcal{M}}$ is $\equiv_{\varphi_{1}}^{\mathcal{M}} \cap \equiv_{\varphi_{2}}^{\mathcal{M}} \cdot{ }^{10}$

Remarks 1.7. (i) Note that the bipartite feature just observed has nothing to do with the current propositional incarnation of supervenience. If we think of $x, y$ as ordinary individuals with properties and of $\varphi$ as a candidate such property, with " $x \equiv_{\varphi} y$ " meaning that $x$ and $y$ are alike in respect of $\varphi$ (i.e., both have or both lack $\varphi$ ), then $\equiv_{\varphi}$ is a bipartite equivalence relation again.

(ii) Note that as with the relations $\equiv_{\varphi}^{\mathcal{M}}$, the corresponding relations used to define $\vdash_{\mathcal{V}}^{\text {svc }}$ above - between valuations (rather than points in a model) $\equiv_{\varphi}$ defined to hold between $v_{1}$ and $v_{2}$ when $v_{1}(\varphi)=v_{2}(\varphi)$ are bipartite (and for the same reason: these are bivalent valuations). $\quad \triangleleft$

We see here a certain disanalogy with between the cases of the binary strict implication, internalizing inference-determined consequence and the binary $D_{1}$ connective internalizing supervenience-determined consequence: for the former, the binary connective suffices for the general many-premiss case (finitely many premisses, at least), since we can conjoin the premisses and reduce this to the one-premiss case (" $\left(\varphi_{1} \wedge \ldots \wedge\right.$ $\left.\varphi_{n}\right)-3 \psi$ "), whereas in the latter case there is nothing that can be done analogous to forming conjunctions which will reduce the general case to the one-premiss case and allow the work of the various $\mathrm{D}_{n}$ to be done just by $D_{1}$.

All of that, however, applies in the general setting in which Fan's discussion locates itself rather than in the special case of Goranko and Kuusisto's discussion, in which $S$ is the universal ternary relation (on the universe of the models under consideration) - or, if one is thinking

10 Further elaboration of this point can be found in [Humberstone, 1992]. As for equivalence relations on the set of possible worlds - or alternatively the corresponding partitions - Lewis [1988] has suggested that these do good useful work as a way of thinking of subject matters. The link to the present material is stressed in [Humberstone, 2002]. In the bipartite case of current interest, the partitioning of the worlds into those verifying a given $\psi$ and the rest, is the subject matter whether or not $\psi$, and in Lewis's terminology its being the case that $\mathrm{D}(\varphi ; \psi)$ would be expressed by saying that this subject matter is part of the subject matter: whether or not $\varphi$; as Example 1.5(i) and the discussion following it shows, this part-of relation is a somewhat degenerate affair in the bipartite case. Applications of, and modifications to, Lewis's general account of subject matter (and aboutness) - which is far from bipartite in the general case - can be found in [Humberstone, 2000] and [Yablo, 2014]. As all these "whether"s suggest, we are here in the vicinity of the semantics of questions on which see Section 6 below. 
of " $S_{w} x y$ " as "Rwx and $R w y$ ", of the case in which $R$ is the universal binary relation. For this case (and indeed whenever $R$ is reflexive), Goranko and Kuusisto observe, one can define an S5 modality $\square$ by setting $\square \varphi={ }_{\text {Df }} \mathrm{D}_{0} \varphi \wedge \varphi$ (the usual definition from the 1960s of necessity in terms of noncontingency ${ }^{11}$ ) and then define $D_{1}$ using this by saying that $\varphi$ 's truth-value settles $\psi$ 's everywhere in the model $;{ }^{12}$ that is, we define $\mathrm{D}_{1}(\varphi ; \psi)$ to be the following, in which, as above, $\square(\varphi \rightarrow \psi)$ is written as $\varphi-3 \psi$, and $\psi$ is said to be strictly equivalent to one of the four 1-ary truth-functions of $\varphi$ :

$$
((\varphi-3 \psi) \vee(\varphi \neg \neg \psi)) \wedge((\neg \varphi-3 \psi) \vee(\neg \varphi \neg \neg \psi))
$$

Alternatively, in the form of a disjunction of strict equivalences - writing the strict biconditional as " $\approx$ " - we can put the definiens like this:

$$
(\psi \approx \varphi) \vee(\psi \approx \neg \varphi) \vee(\psi \approx \top) \vee(\psi \approx \perp) .
$$

This last is the approach taken in [Goranko and Kuusisto, 2018, Proposition 3.3], though, in keeping with the multigrade treatment, they in effect define all the various $\mathrm{D}_{k}\left(\varphi_{1}, \ldots, \varphi_{k} ; \psi\right)$ simultaneously. When the definiens is written in the style of (1.4), for $\mathrm{D}_{k}$ there will be $2^{2^{k}}$ disjuncts as we run through all the truth-functional compounds of $\varphi_{1}, \ldots, \varphi_{k}$, making these, as Goranko and Kuusisto remark, non-polynomial definitions. ${ }^{13}$ A similar situation arises with an incarnation of supervenience for predicate symbols relative to first-order theories, in which what corresponds to the $\square$ hidden in the above " $\approx$ " notation is an ordinary universal

11 This definition works in the setting of any normal modal logic in which every formula of the form $\square \varphi \rightarrow \varphi$ is provable; if that condition is not met some definition of necessity in terms of noncontingency may be still be available, though in general this is not so. On these two points, see [Cresswell, 1988; Humberstone, 1995a]. More generally, see the index entries under 'contingency (and noncontingency)' in [Humberstone, 2016]. The definition cited of necessity as noncontingency conjoined with truth perhaps first appeared in [Montgomery and Routley, 1966]; the following year saw the corresponding move ( $\forall$ corresponding to $\square$ ) in classical predicate logic: Thomason and Leblanc Thomason and Leblanc.

12 In any point-generated model, that is; the first conjunct of the formula displayed below says concerning $\varphi$ that its truth settles $\psi$ 's truth-value either positively or negatively, while the second conjunct says the same for $\neg \varphi$.

13 A definition which is somewhat reminiscent of this was given in [Väänänen, 2008 , p. 202] working in essentially what Goranko and Kuusisto call $\mathcal{D}$, though the interpretation of the $\vee$ linking the cases in the definiens - which Väänänen also notes grows exponentially in $k$-is rather different, as we shall see in Section 3. The binary $\mathrm{D}_{1}$ can only have sentence letters in its scope and $\mathrm{D}_{1}(p, q)$ is defined to be $\left(p \wedge \mathrm{D}^{0} \mathbf{q}\right) \vee$ 
quantifier. (1.4) then corresponds to (8) or (9) of [Humberstone, 1998a, p. 246], where this connection is noted between supervenience and the standard model-theoretic notion of piecewise definability. If we are considering supervenience for predicates, however, we may want to consider not replacing the necessity operator with a universal quantifier but using a disjunction like (1.4), with each disjunct both universally quantified and necessitated, in which case, as will be recalled in Section 8, we areas Fan [2016] observes - essentially in the area of Jaegwon Kim's observations [see Kim, 1984b; Kim, 1984a, §1, and elsewhere] concerning strong supervenience and reducibility. We pick up this theme in Section 8 below.

\section{Matters Arising}

Three additional points arising from Section 1 calling for comment though not needed for subsequent sections are included here, as a kind of appendix to that section before we move on to the logic to which Goranko and Kuusisto argue their $\mathcal{L}_{\mathrm{D}}$ is superior. First the Example 1.1(i)-inspired D-formula mentioned above,

$$
\mathrm{D}(\varphi ; \psi) \rightarrow(\mathrm{D} \psi \vee \mathrm{D}(\psi ; \varphi))
$$

would not be valid on the general semantics of [Fan, 2016], with the ternary relation $S$ not restricted to the special case in which for some binary relation $R$ we have for all $w, x, y S_{w} x y$ iff $R w x$ and $R w y$. That is, we cannot rule out the possibility that for some Fan model $\mathcal{M}=$ $\langle W, S, V\rangle$ and some $w \in W$, we have
(1) $\mathcal{M}, w \models \mathrm{D}(\varphi ; \psi)$
(2) $\mathcal{M}, w \not \models \mathrm{D} \psi$
(3) $\mathcal{M}, w \not \models \mathrm{D}(\psi ; \varphi)$.

(3) tells us that for some $x, y \in W$ with $S_{w} x y$ we have $x \equiv_{\psi}^{\mathcal{M}} y$ but not $x \equiv_{\varphi}^{\mathcal{M}} y$. (2) tells us that for some $u, v \in W$ with $S_{w} u v$, not $u \equiv_{\psi}^{\mathcal{M}} v$. If $S$ were related to a binary $R$ as above we would reason that from the gloss just given on (3) $x$ and $y$ are $R$-successors of $w$ which agree on $\psi$ (in $\mathcal{M})$ but do not agree on $\varphi$, and from the gloss on (3) that one or other of the $R$-successors $u, v$ of $w$ which disagree on $B$, so select whichever of them disagrees with $x$ and $y$ on $\psi$ that is, and the selected point must give $\varphi$ the same truth-value that $x$ does or else the same truth-value that $y$ does. In the former case the selected point together with $x$ provides

$\left(\neg p \wedge \mathrm{D}_{0} q\right)$, when translated into the present notation. In Section 4 , the general form of Goranko and Kuusisto's definition will be described, rather than just the $k=1$ case. 
a counterexample to (1), being $R$-successors of $w$ which agree on $\varphi$ but not on $\psi$, and in the latter case it is the selected point together with $y$ which provide such a counterexample.

For this reasoning to go through using the ternary relation we should need the following condition, in which for simplicity $S_{w}$ is written as a binary relation $T$ (since, there being no embedding of $D$ within the scope of $\mathrm{D}$, this $w$ can be taken as fixed for the evaluation of our formula), and the whole thing is to be understood with all variables bound by universal quantifiers:

$$
(T x y \wedge T u v) \rightarrow(T x v \wedge T y v \wedge T x u \wedge T y u)
$$

and this universally quantified condition is easily seen to be equivalent to the conjunction of two claims about the relation (signified by) $T$, the first being that this relation is symmetric and the second being that it satisfies the following absent-minded variant on transitivity: $\forall x, y_{1}, y_{2} z\left(\left(T x y_{2} \wedge\right.\right.$ $\left.\left.T y_{1} z\right) \rightarrow T x z\right)$-absent-minded because it forgets to require that $y_{1}=$ $y_{2}$. This last condition taken by itself, is equivalent to the second-order condition there are subsets $X, Y$ of the domain such that for all $x, y, T x y$ iff $x \in X$ and $y \in Y$. When we add the further condition that $T$ is symmetric, this means that the promised subsets coincide: the relation in question holds between them just in case both are elements of the set in question [see Humberstone, 1995b, Proposition 7(i), p. 127]. And what is that set, in the present instance, recalling that "Txy" just abbreviates $S_{w} x y$ for some given $w$ in one of the current models? It is of course just $R(w)$ - and if we didn't start with $R$ and define $S_{w} x y$ as $R w x \wedge R w y$ we can, when these two conditions are satisfied, retrospectively introduce an $R$ for which such and equivalence holds by putting $R w x$ iff $S_{w} x x$ (equivalently, iff $\exists y\left(S_{w} x y\right)$ ). Summing up, then for the formula with which we began, no restriction on Fan's $S_{w} x y$ short of than one making this equivalent to $R z w \wedge R w y$ for some binary $R$ will validate the formula, though of course $R$ need not be the universal relation on the models, as in Goranko and Kuusisto's semantics for $\mathcal{L}_{\mathrm{D}}$.

Coming to the second of our three points: there is an interesting difference between, on the one hand, the formula we have been considering - alternatively the consequence-relational formulation

$$
\mathrm{D}(\varphi ; \psi) \vdash \mathrm{D} \psi \vee \mathrm{D}(\psi ; \varphi),
$$

which we observed on the basis of the reasoning behind Example 1.5(i) to hold for $\vdash$ taken as the $\models$ of (D1.1) on p. 6 , and on the other hand, the 
metalinguistic formulation in Example 1.5(i) itself, where we had no special connectives but deployed the apparatus of supervenience-determined consequence relations. Rather, the claim was that for any such consequence relation $\vdash$, one has (for all $\varphi, \psi$ in its language):

$$
\text { If } \varphi \vdash \psi \text { then either } \vdash \psi \text { or } \psi \vdash \varphi \text {. }
$$

This can be used to show the kind of trouble an analogue of conjunction for supervenience determined consequence relations would cause from the discussion of bipartite equivalence relations in Section 1 (preceding Remarks 1.7, and in (ii) thereof), so we know that a connective $\circ$ satisfying for all $\varphi, \psi$ in the language of $\vdash$ supervenience determined by some $\mathcal{V}$ :

$$
(\circ 1) \varphi \circ \psi \vdash \varphi \quad(\circ 2) \varphi \circ \psi \vdash \psi \quad(\circ 3) \varphi, \psi \vdash \varphi \circ \psi \text {. }
$$

We know that there would be trouble from having a $\circ$ satisfying these three condition since they would mean we had $\Gamma, \varphi, \psi \vdash \chi$ iff $\Gamma, \varphi \circ \psi \vdash$ $\chi$ which even for $\Gamma=\varnothing$ would run into the difficulty noted in Section 1 - the disanalogy between $D_{1}$ and $3-$ about intersections of bipartite equivalence relations not being (in general) bipartite. But even just ( $\circ 1)$ and (o2) would have a trivializing effect. ${ }^{14}$ For, invoking the principle from Example 1.5(i) taking (o1) as its antecedent, we conclude that

$$
\text { For all } \varphi, \psi: \vdash \varphi \text { or else } \varphi \vdash \varphi \circ \psi \text {, }
$$

and with the help of (०2) on the second disjunct here, we conclude that

$$
\text { For all } \varphi, \psi: \vdash \varphi \text { or else } \varphi \vdash \psi \text {. }
$$

So we have derived from (o1) and (o2) the conclusion that for any formulas $\varphi$ and $\psi$, either all valuations in any $\mathcal{V}$ supervenience determining $\vdash \operatorname{assign} \varphi$ the same truth value, for else any two that assign the same truth-value to $\varphi$ assign the same truth-valued to $\varphi$.

This is of course not the case for arbitrary $\mathcal{V}$, illustrating the nonconservative effect (since $\varphi, \psi$ are arbitrary and need not themselves feature $\circ$ ) of (०1) and (०2). Indeed, for a more symmetrical formulation of the difficulty we can re-invoke the principle of Example 1.5(i) again to the " $\varphi \vdash \psi$ " part of our conclusion, giving us the following general conclusion:

$$
\text { For all } \varphi, \psi: \vdash \varphi \text { or } \vdash \psi \text { or } \varphi \dashv \vdash \psi \text {. }
$$

14 Humberstone [1992, pp. 133-136] also makes trouble for this pair of conditions, in a slightly different way. 
Of course " $\varphi \dashv \vdash$ " is just short for " $\varphi \vdash \psi$ and $\psi \vdash \varphi$ " - something that when we are concentrating on inference determination would naturally be read by saying that $\varphi$ and $\psi$ are equivalent (according to $\vdash$ ), though that formulation would be potentially confusing here since this relation does not mean that $\varphi$ and $\psi$ always assume the same truth-value (on the valuations of interest) but rather than each determines the other's truth-value over the class of such valuations (as do, for example, any formula and its negation over the class of Boolean valuations ${ }^{15}$ ). For this reason the term equivenience, rather than equivalence, was used in [Humberstone, 1992] for this relation.

Since we will want to appeal to it as a lemma for the proof of an observation in Section 8 (Proposition 8.2), we include here without proof an easy observation on equivenience, using the notation $-\Vdash$ abbreviating the conjunction of a $\vdash$-claim (having one formula on the left) with its converse, and omitting any super- or subscripts from the "-l" part of the notation.

Proposition 2.1. Whenever for a class $\mathcal{V}$ of valuations for a language with $\varphi, \psi$ among its formulas, $\neg$ as one of its connectives, and $\mathcal{V}$ containing only $\neg$-Boolean connectives, we have $\varphi-\nVdash_{\mathcal{V}}^{\text {svc }} \psi$, we have either $\varphi \dashv \nVdash_{\mathcal{i n f}} \psi$ or else $\varphi \dashv \nVdash_{\mathcal{V}}^{\text {inf }} \neg \psi$.

In $(*)-(* * *)$ we have a (metalinguistic) universal quantifier with broader scope than the disjunction "or", but if $\vdash$ is a substitutioninvariant ${ }^{16}$ consequence relation then we can trade this in for a (typically) stronger claim in the form of a disjunction of universally quantified claims, which we may call $(\dagger)-(\dagger \dagger \dagger)$ :

Either for all $\varphi, \psi: \vdash \varphi$ or else for all $\varphi, \psi: \vdash \varphi \circ \psi$.

Either for all $\varphi: \vdash \varphi$ or else for all $\varphi, \psi: \varphi \vdash \psi$.

Either for all $\varphi: \vdash \varphi$ or else for all $\varphi, \psi: \varphi \dashv \Vdash \psi$.

15 Or more precisely over the broader class of $\neg$-Boolean valuations, by which is meant valuations $v$ such that for all formulas $\varphi, v(\neg \varphi)=T$ iff $v(\varphi)=F$. This is analogous to the notion of a $\leftrightarrow$-Boolean valuation defined in Section 1. It is natural, having isolated these and corresponding notions for each connective \# under discussion, to call a valuation Boolean tout court when it is \#-Boolean for all the \# concerned.

16 A consequence relation $\vdash$ is substitution-invariant (also called structural) if whenever $\Gamma \vdash \varphi$, we have $s(\Gamma) \vdash s(\varphi)$ for any substitution $s$ (mapping sentence letters to formulas), where $s(\Gamma)$ is $\{s(\psi) \mid \psi \in \Gamma\}$. 
This would obviously be bad news for our $\vdash$ satisfying (o1) and (o2), but how likely is it that our supervenience-determined $\vdash$ is substitutioninvariant? We return to this question after interpolating an example suggested by our findings so far for the generalized superveniencedetermined consequence relations mentioned in Remark 1.3(i).

EXAMPlE 2.2. Having seen the trouble $(\circ 1)$, ( $\circ 2)$ and- $(\circ 3)$ can cause for a supervenience determined consequence relation we might naturally consider what happens if we weakening the first two of these conditions, as we may in the setting of the generalized consequence relations $\Vdash_{\mathcal{V}}^{\text {svc }}$ of Remark 1.3(i); writing simply "Iト" for some such relation this would be the following condition (understood as applying for arbitrary formulas $\varphi, \psi)$ :

$$
\text { (०4) } \varphi \circ \psi \Vdash \varphi, \psi \text {. }
$$

One might naturally come to enquire as the fate of such a condition motivated by issues of duality, since it is in effect the converse - leftright reversal - of the condition ( $\circ 3)$, satisfied by every binary Boolean connective. (See the discussion of (2.1) below.) ( $\circ 4$ ) tells us this about the underlying $\mathcal{V}$ : any valuations in $\mathcal{V}$ which disagree on the compound $\varphi \circ \psi$ must disagree on both components, $\varphi$ and $\psi$. But, as we shall now show, the only way for this to be the case is for the binary truth-function associated with $\circ$ over $\mathcal{V}$-relying here on the obvious understanding of this phrase, explicitly defined before (2.1) below - to fail to be essentially binary (that is, it must be the first projection, second projection, or constant true truth-function or else the negation of one of these three). For, taking $\varphi, \psi$ as $p, q$ respectively, if $u, v \in \mathcal{V}$ differ on $p, q$, then $u(p)$ and $v(p)$ must be $T$ and $F$ or vice versa, and $u(q)$ and $v(q)$ must be $T$ and $F$ or again vice versa, and either way we must have, using "०" denote the function associated with the connective $\circ$ over $\mathcal{V}$ : :

$$
\text { (1) } T \circ T \neq F \circ F \text { and (2) } T \circ F \neq F \circ T \text {. }
$$

Here (1) arises from the evaluation of $p \circ q$ for the case of $u(p)=T \neq$ $v(p)=F$ and $u(q)=T \neq v(q)=F$, so we need $u(p \circ q) \neq v(p \circ q)$, if (०4) is to be satisfied for $\Vdash$ as $\Vdash_{\mathcal{V}}^{\text {svc }}$. And (2) arises similarly from the case in which $u(p)$ and $u(q)$ differ. We are now in a position to give a schematic description of the truth-table depicting the behaviour of the truth-function $\circ$, letting $\xi$ be whichever element of $\{T, F\} T \circ T$ is, with $\bar{\xi}$ for the other truth-value, and $\zeta$ be whichever value $T \circ F$ is (again, with $\bar{\zeta}$ for its complement). 


\begin{tabular}{lll}
$p$ & $\circ$ & $q$ \\
\hline$T$ & $\xi$ & $T$ \\
$T$ & $\zeta$ & $F$ \\
$F$ & $\bar{\zeta}$ & $T$ \\
$F$ & $\bar{\xi}$ & $F$
\end{tabular}

The overbarring in the value for the fourth line is there because this must differ from the value in the first line because of (1), and in the case of the third line, given the second, because of (2). Given the bivalent setting, $\zeta$ must coincide with $\xi$ or else with $\bar{\xi}$. In the first of these cases the main column reads (top to bottom) $\xi, \xi, \bar{\xi}, \bar{\xi}$, and the function $\circ$ does not vary with its second (and therefore inessential) argument. ${ }^{17}$ In the second case, the main column becomes $\xi, \bar{\xi}, \xi, \bar{\xi}$, and this time it is the first argument that is inessential. Either way, the associated truth-function cannot be any one of the ten essentially binary truthfunctions.

Substitution-invariance is typically taken as a desideratum for a consequence relation purporting to tell us what follows from what on formal grounds, and so we typically make $\mathcal{V}$ large enough not to privilege the sentence letters in any way when it comes to considering the consequence relation inference determined by $\mathcal{V}$ : any sequence of truth-values that formulas $\varphi_{1}, \ldots, \varphi_{n}$ might have should be a sequence that any $n$-termed sequence of sentence letters $q_{1}, \ldots, q_{n}$ can assume. The following proof

17 I avoid the usual way of putting this: "... does not depend on its second argument" to minimize any risk of confusion, since in the dependence logic literature even the projection-to-the-first-argument two-place function depends on the set of both of its arguments, since dependence on a subset implies dependence on any superset. This is because, putting the matter epistemically, dependence of one thing on a second amounts to the knowledge of latter's being sufficient for knowledge of the former, rather than its being necessary. Relatedly, we have the 'at most' construction in glossing, e.g., $\forall x \forall y \exists z \Phi(x, y, z)$ as saying that for all $x$ and $y$ there is a $z$ depending at most on $x$ and $y$, such that $\Phi(x, y, z)$; since this formula will be true in any structure verifying $\forall x \exists z \forall y \Phi(x, y, z)$, it cannot be required that $z$ in the former case depends on both $x$ and $y$ in the sense in which a two-place function which is essentially binary depends on both of its arguments. Similarly if a policy has already been agreed on by those in power, one would not normally say "Whether the policy is adopted depends on - or is determined by - the result of the popular vote," and one would not normally expect to persuade anyone by elaborated: "And this is how it depends on that result. If the vote is in favour, the policy will be adopted, and if the vote is against, the policy will be adopted." For more in this vein, see note 1 of [Ciardelli, 2016b] (or note 3 of Chapter 1 of [Ciardelli, 2016a]. 
adapts the observation of Goranko and Kuusisto from the end of Section 1 above, about reducing $\mathrm{D}$ - or as in our exposition, the various $\mathrm{D}_{k}-$ to strict equivalence (with a universal $\square$ understood). This appeared for a simple special case in (1.4) at the end of that section, though in fact we follow more closely the formulation using strict implication in (1.3) there. To stay close to that formulation, we assume we have $\neg$ as a connective and that it behaves classically, which is the effect of the condition of $\mathcal{V}$ (the notion of a $\neg$-Boolean valuation having been defined in note 15$)$ :

Proposition 2.3. Let $\mathcal{V}$ be any class of valuations all of which are $\neg$-Boolean. If $\vdash \mathcal{V}$ inf is substitution-invariant, then $\vdash \stackrel{\mathcal{V}}{\text { svc }}$ is substitutioninvariant.

Proof. Each claim to the effect that $\varphi_{1}, \ldots, \varphi_{n} \vdash \stackrel{\mathcal{V}}{\text { svc }} \psi$ is equivalent to a combination of claims involving $\vdash$ inf as we illustrate with the case of $n=2$. The claim, for a given $\varphi_{1}, \varphi_{2}, \psi$ we have $\varphi_{1}, \varphi_{2} \vdash \mathcal{V}$ svc $\psi$ is easily seen to be equivalent to the following claim:

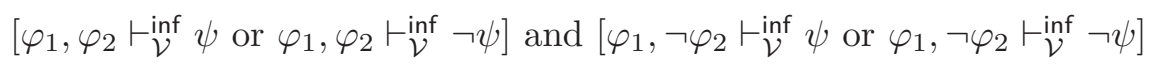

and

$$
\begin{aligned}
& {\left[\neg \varphi_{1}, \varphi_{2} \vdash \mathcal{V} \text { inf } \psi \text { or } \neg \varphi_{1}, \varphi_{2} \vdash \mathcal{V} \text { inf } \neg \psi\right] \text { and }} \\
& {\left[\neg \varphi_{1}, \neg \varphi_{2} \vdash \text {-inf } \psi \text { or } \neg \varphi_{1}, \neg \varphi_{2} \vdash \underset{\mathcal{V}}{\text { inf }} \neg \psi\right] \text {. }}
\end{aligned}
$$

By the assumed substitution-invariance of $\vdash$ inf , we infer a fourfold conjunction of disjunctions of which the second, by way of illustration, would be the following:

for all $s, s\left(\varphi_{1}\right), \neg s\left(\varphi_{2}\right) \vdash \underset{\mathcal{V}}{\inf } s(\psi)$ or for all $s, s\left(\varphi_{1}\right), \neg s\left(\varphi_{2}\right) \vdash \vdash_{\mathcal{V}}^{\inf } \neg s(\psi)$.

Here we take the liberty of rewriting, e.g., " $s\left(\neg \varphi_{2}\right)$ " as " $\neg s\left(\varphi_{2}\right)$ ". This makes it clear that the fourfold conjunction in question amount to the claim that

$$
\text { for all substitutions } s: s\left(\varphi_{1}\right), s\left(\varphi_{2}\right) \vdash \mathcal{V} \text { svc } s(\psi) \text {, }
$$

completing our proof by consideration of a representative case.

We return to the contrast between, on the one hand, the D-formulation corresponding to $(* *)$ :

$$
\mathrm{D}(\varphi ; \psi) \vdash \mathrm{D} \psi \vee \mathrm{D}(\psi ; \varphi),
$$

with $\vdash$ as the relation $\models$ defined in (D1.1) on p. 6 and, on the other, the D-free formulations with supervenience determined $\vdash$. In the later 
setting, (॰1) and (०2) become $\vdash \mathrm{D}(\varphi \circ \psi ; \varphi)$ and $\vdash \mathrm{D}(\varphi \circ \psi ; \psi)$. The former gives us the left hand formula in the following special case of the $\vdash$-statement inset just above:

$$
\mathrm{D}(\varphi \circ \psi ; \varphi) \vdash \mathrm{D} \varphi \vee \mathrm{D}(\varphi \circ \psi ; \varphi),
$$

so we conclude that $\vdash \mathrm{D} \varphi \vee \mathrm{D}(\varphi \circ \psi)$, and now using the 'transitivity' fact that - using new of schematic letters to avoid confusion $\mathrm{D}(\alpha ; \beta), \mathrm{D}(\beta ; \gamma) \vdash \mathrm{D}(\alpha ; \gamma)^{18}$ and our current analogue of (o2), we conclude that $\vdash \mathrm{D} \varphi \vee \mathrm{D}(\varphi ; \psi)$. But note that now we cannot use a representative instance $\mathrm{D} p \vee \mathrm{D}(p ; q)$ together with substitution invariance in order to infer, by analogy with the earlier $(\dagger \dagger)$ that for all $\varphi, \psi, \chi$, $\mathrm{D} \chi \vee \mathrm{D}(\varphi ; \psi)$. (This would correspond to an available reformulation of $(\dagger \dagger)$ in which the first disjunct is "for all $\chi: \vdash \chi$ ".) This is of course because the current logic does not enjoy the Disjunction Property, so we cannot make the required substitutions, and so the present contrast is only to be expected, but it does leads us naturally to our third and final point of commentary on the concepts introduced in Section 1.

This third point again concerns substitutions and disjunctions, and to the definability of the various $\mathrm{D}_{k}$ in terms of $\mathrm{D}_{0}$ at the end of Section 1 , and the illustrative $k=1$ case there mentioned will be repeated presently. Before getting to that, however, we need to back up and recall some notions [e.g., from Humberstone, 2011, Chapter 3]. If $v$ is a valuation for some language with an $n$-ary connective \# and $f$ is an $n$-ary (bivalent) truth-function, we say that $f$ is associated with \# on $v$ if for any formulas $\varphi_{1}, \ldots, \varphi_{n}$ of the language in question $v\left(\#\left(\varphi_{1}, \ldots, \varphi_{n}\right)\right)=f\left(v\left(\varphi_{1}\right), \ldots, v\left(\varphi_{n}\right)\right)$. Then we call the $n$-ary connective \# pseudo-truth-functional over $\mathcal{V}$ iff for every $v \in \mathcal{V}$ there exists an $n$-ary truth-function $f$ such that $f$ is associated with \# on $v$, and we call \# truth-functional over $\mathcal{V}$ if there is some $n$-ary truth-function $f$

18 As in (11) under Example 1.4 in [Väänänen, 2008] and Proposition 3(ii) of [Fan, 2016]. The text to which this note is appended has 'transitivity' with scare quotes because, strictly, a connective is not the kind of thing-even when further relativized to a particular logic-can be transitive or otherwise; however, it is not hard to find a pertinent associated binary relation which can be (and in the present case is) [see Humberstone, 2011, 3.34]. In addition we should note that the internalized version of the 'cut' condition on consequence relations is not given simply by " $\mathrm{D}(\alpha ; \beta), \mathrm{D}(\beta ; \gamma) \vdash \mathrm{D}(\alpha ; \gamma)$ " but rather, more generally, by: $\mathrm{D}\left(\alpha_{1}, \ldots, \alpha_{m} ; \beta\right), \mathrm{D}\left(\delta_{1}, \ldots, \delta_{n}, \beta ; \gamma\right) \vdash \mathrm{D}\left(\alpha_{1}, \ldots, \alpha_{m}, \delta_{1}, \ldots, \delta_{n} ; \gamma\right)$, the simple version being the special case in which $m=1, n=0$. 
such that for all $v \in \mathcal{V}, f$ is associated with \# on $v \cdot{ }^{19}$ Compare now the following two conditions on $\mathcal{V}$, concerning which we assume, for ease of exposition as in Section 1, that all valuations in $\mathcal{V}$ are $\leftrightarrow$-Boolean, with $\leftrightarrow$ a connective of the language concerned:

$$
\begin{gathered}
\varphi_{1}, \ldots, \varphi_{n} \vdash \mathcal{V} \text { 战 } \#\left(\varphi_{1}, \ldots, \varphi_{n}\right) \\
\varphi_{1} \leftrightarrow \psi_{1}, \ldots, \varphi_{n} \leftrightarrow \psi_{n} \vdash \stackrel{\mathcal{V}}{\text { inf }} \#\left(\varphi_{1}, \ldots, \varphi_{n}\right) \leftrightarrow \#\left(\psi_{1}, \ldots, \psi_{n}\right)
\end{gathered}
$$

Given the restriction to $\leftrightarrow$-Boolean valuations (which could be avoided with some circumlocution [see Humberstone, 2011, 3.23]) the extensionality condition (2.2) says that \#-compounds with components having the same truth-values (on an arbitrary $v \in \mathcal{V}$ ) themselves have the truthvalue (on $v$ ), which is equivalent to saying that \# is pseudo-truth-functional over $\mathcal{V}$. (2.1) makes the stronger claim that \# is truth-functional over $\mathcal{V}$. (2.1) in fact represents the general case of supervenience-determined consequence in that, by courtesy of Proposition 1.2, we can strengthen the claim it makes to the following, on the assumption that we have a functionally complete stock of primitive connectives: for all formulas $\varphi_{1}, \ldots, \varphi_{n}, \psi$

$$
\varphi_{1}, \ldots, \varphi_{n} \vdash \vdash_{\mathcal{V}}^{\text {svc }} \psi \text { iff } \psi-\vdash_{\mathcal{V}}^{\text {inf }} \#\left(\varphi_{1}, \ldots, \varphi_{n}\right),
$$

for some (not necessarily primitive) $n$-ary Boolean connective $\# .{ }^{20}$ (2.1)style formulations are emphasized in [Humberstone, 2002, p. 96], where the label "(\# Composition)" is used for them; the condition ( $\circ 3)$ above was the $n=2$ incarnation of this condition, with \# as $\circ$. The simple binary contrast between truth-functionality and pseudo-truth-functionality mutates into something more multifarious in the setting of the Kripke semantics for (esp. normal) modal logics, as we shall see in a moment.

19 Note that in neither case is there a requirement that there should be exactly one truth-function associated with \# on a given $v$. For example, take the valuation $v_{T}$ assigning the value $T$ to every formula and \# is any one-place connective, then associated with \# on $v_{T}$ are both the constant-true truth-function and the identity truth-function.

20 The functional completeness condition can probably be dropped here, though the author does not have a general proof to that effect. Here is a simple example: where the only primitive connective is $\neg$ we have and $\mathcal{V}$ is the class of $\neg$-Boolean valuations we have not only $p \vdash \mathcal{V}$ svc $\neg p$, literally instantiating (2.1), but also $\neg p \vdash_{\mathcal{V}}^{\text {svc }} p$, here taking \# as $\neg$ in the inset generalization of (2.1): but of course ex hypothesi we already have $\neg$ in the present (far from functionally complete) language. 
First, we need to back up a little and notice a comment from [Humberstone, 1986, p. 34], where apropos of (2.2) - differently notated and with the $n=1$ case in focus, the following appears. In this quotation the passage is reworded to suit the general case and reformulated into the present notation. The reference to not reducing the truth-value of a compound to those of its components is a reference to the possible failure of a pseudo-truth-functional connective to be truth-functional, relative to a given class of $\leftrightarrow$-Boolean valuations:

It seems to be pertinent at this point to remark that in spite of the impossibility of reducing the question of $\#\left(\varphi_{1}, \ldots, \varphi_{n}\right)$ 's truth-value to the truth-values of the $\varphi_{i}$, we do have, and always will have, when (2.2) is satisfied, a case of supervenience: for what (2.2), for $n=0$, says is precisely that the truth-values of $\#\left(\varphi_{1}, \ldots, \varphi_{n}\right)$ and $\#\left(\psi_{1}, \ldots, \psi_{n}\right)$ cannot differ without a difference between those of some $\varphi_{i}$ and $\psi_{i}$ (for at least one $i, 1 \leq i \leq n)$.

What may be of interest here is the contrast with the account of supervenience (or determination) from Section 1 above. For simplicity, as in that section, let us look at the 1-place case. At the end of Section 1 we had the Goranko-Kuusisto disjunction (1.4) repeated here as (2.3), with " $\approx$ " written explicitly as a necessitated biconditional, and treating the case in which $\varphi$ is $p$ and $\psi$ is \#p for a one-place connective \#:

$$
\vdash \square(\# p \leftrightarrow p) \vee \square(\# p \leftrightarrow \neg p) \vee \square(\# p \leftrightarrow \top) \vee \square(\# p \leftrightarrow \perp)
$$

(One would normally write just "\#p" and " $\neg \# p$ " for $\# p \leftrightarrow \top$ and $\# p \leftrightarrow \perp$, but we persist with the longer format to emphasize that we are running through the biconditionals with representatives of the 1-ary Boolean compounds of $p$ in the right.) Thus on this account of what it is for $\# q$ to be supervenient on (dependent on or determined by) $q$, relative to some class of $\vee$ - and $\leftrightarrow$-Boolean valuations is given by the truth of (2.3) on every valuation in the class. In a logical setting, the usual considerations favouring closure under Uniform Substitution would have one want this to hold not just for $p$ but for all formulas $\varphi$, and this would constitute the logic in question's telling us that \# forms compounds supervenient on their components. ${ }^{21}$ But this is not what is

${ }^{21}$ In Section 6 we will encounter a logic defiantly not closed under Uniform Substitution; many such logics - assuming this word is not defined so as to require substitution-invariance - from the literature are listed on p. 192 of [Humberstone, 2011]. 
for a \#-compound to be supervenient on its component(s) according to the proposal just quoted from [Humberstone, 1986], which is instead the following ( $p, q, r, s$ being distinct sentence letters):

$$
\vdash(\# p \leftrightarrow p) \vee(\# q \leftrightarrow \neg q) \vee(\# r \leftrightarrow \top) \vee(\# s \leftrightarrow \perp)
$$

The change of sentence letter (propositional variable) from disjunct to disjunct, which makes for Halldén incompleteness if the logic tells us nothing more informative about \#, is required to capture the idea that on each valuation $v$ in any class of valuations $\mathcal{V}$ for which $\vdash$ is $\vdash \stackrel{\text { inf }}{\text {, \# is }}$ associated with a truth-function: this has to be the same truth-function for all formulas to which \# is applied, even though which function that is can vary from one $v$ to another. ${ }^{22}$ (The equivalence of (2.3) with the set of instances of (2.2) for $n=1$, for $\mathcal{V}$ containing only $*$-Boolean valuations for $* \in\{\leftrightarrow, \neg, \vee, T, \perp\}$ is noted in [Humberstone, 1986]. A treatment of these matters purified of the Boolean connectives is given in [Humberstone, 1997].) Note that without the change of variables from disjunct to disjunct - i.e., if we just have (2.3) without the occurrences of $\square$, we just have substitution instance (in the language with \#) of a Boolean tautology, rather than a constraint with any bite to it - as would be the case even with the first two disjuncts discarded, or the last two. While we are considering the contrast between (2.3) and (2.4), which should fill out the picture by adding into he mix a condition combining the variable-changing aspects of (2.4) with the original necessitation-ofdisjunctions feature of (2.3):

$$
\vdash \square(\# p \leftrightarrow p) \vee \square(\# q \leftrightarrow \neg q) \vee \square(\# r \leftrightarrow \top) \vee \square(\# s \leftrightarrow \perp)
$$

To compare (2.3)-(2.5) from the perspective of model-theoretic semantics in which, as for Goranko and Kuusisto, $\square$ is interpreted as quantifying over all points a model $\mathcal{M}$, i.e. over the universe of $\mathcal{M}$, which we shall denote by $U(\mathcal{M}), f$ and $\varphi$ will be understood as ranging over arbitrary one-place truth-functions - since we continue to illustrate with \# 1-ary

${ }^{22}$ One could even write (2.4) in the same way as (2.3) but with a substitutional reading of " $\square$ " - a sort of oversimplified version of McKinsey [1945] [see Humberstone, 2016 , pp. 165-169, for further references and discussion] - so that $\square \varphi$ is true at a point in a model if every $\square$-free substitution instance of $\varphi$ is true at that point. Here the " $\square$-free" condition is included to avoid an impredicativity, but the suggestion remains fraught with danger. For example, $\square(p \vee q) \rightarrow(p \vee r)$ will be valid but the result of (uniformly) substituting $q \rightarrow q$ for $q$ in this formula will not be. 
as above - and arbitrary formulas. For such an $\mathcal{M}$ with $x \in U(\mathcal{M})$ we write $v_{x}^{\mathcal{M}}$ for the valuation defined by: $v_{x}^{\mathcal{M}}(\varphi)=T$ iff $\mathcal{M}, x \models \varphi$. Note that nothing has been said as to how models as currently envisaged deal with the connective \#, though the discussion presumes that the truthvalue if $\varphi$ and $\psi$ are true at the same points in a model, so are \# $\varphi$ and $\# \psi$, and also that the consequence relation inference-determined by $\left\{v \mid v=v_{x}^{\mathcal{M}}\right.$ for some $\left.\mathcal{M}, x \in U(\mathcal{M})\right\}$ is substitution-invariant. Corresponding to the syntactic conditions (2.3)-(2.5) we have the following semantic conditions:

$$
\begin{array}{ll}
\text { for (2.3): } & \forall \varphi \forall \mathcal{M} \exists f \forall w \in U(\mathcal{M}) \cdot v_{w}^{\mathcal{M}}(\# \varphi)=f\left(v_{w}^{\mathcal{M}}(\varphi)\right) \\
\text { for (2.4): } & \forall \mathcal{M} \forall w \in U(\mathcal{M}) \exists f \forall \varphi \cdot v_{w}^{\mathcal{M}}(\# \varphi)=f\left(v_{w}^{\mathcal{M}}(\varphi)\right) \\
\text { for (2.5): } & \forall \mathcal{M} \exists f \forall w \in U(\mathcal{M}) \forall \varphi \cdot v_{w}^{\mathcal{M}}(\# \varphi)=f\left(v_{w}^{\mathcal{M}}(\varphi)\right)
\end{array}
$$

Thus for (2.3) the promised $f$ depends on the formula to which it applies as well as on the model under consideration, but within the model it remains fixed from point to point. For (2.4) $f$ remains fixed for all formulas, though its choice depends on the model and the point therein. And for (2.5), while again uniform for different formulas, $f$ depends only on the model, being invariant across points within that model. It is perhaps not necessary to decide which of these candidates best deserves to be regarded as cashing out the idea of the a connective's forming compounds supervenient on its components, especially since there is probably no single intuitive idea answering to this description, for which reason here we rest content with a comparative observation.

To illustrate the contrast between, in particular, (2.3) and (2.4), let us work with models $\mathcal{M}=\langle W, X, V\rangle$ in which as usual $W \neq \varnothing$ and $X \subseteq W$, and a language with, alongside a functionally complete stock of Boolean connectives standardly interpreted and the operator $\square$ with accessibility relation $W \times W$ (as in Goranko and Kuusisto), two additional (1-ary) sentence operators $O_{1}, O_{2}$ interpreted thus, with $\mathcal{M}$ as above, for all formulas $\varphi$, for arbitrary $x \in W$ :

$\mathcal{M}, x \models O_{1} \varphi$ if and only if $\mathcal{M}, x \models \varphi$ iff $x \in X$.

$\mathcal{M}, x \models O_{2} \varphi$ if and only if $\mathcal{M}, x \models \varphi$ iff $\|X\|>3$.

Then we have (2.4) satisfied by the associated local consequence relation $\vdash$ whether \# is taken as $O_{1}$ or as $O_{2},{ }^{23}$ since in either case this is so for the

${ }^{23}$ By the associated local consequence relation is meant the consequence relation inference-determined by the class of all valuations of the form $v_{x}^{\mathcal{M}}$ in which truth at a point in a model is defined as stipulated here. 
subdisjunction consisting of the first two disjuncts of the formula there: at each point any a model the operator concerned ends up expressing the identity truth-function or the negation truth-function. But only for \# as $\mathrm{O}_{2}$ is (2.5) satisfied (as it is again in virtue of the initial 2term subdisjunction), since only in that case is the truth-function fixed throughout the model chosen. This has a consequence shared with \# satisfying (2.3) but not with (2.4): at any given point in a model any formula $\# \varphi$ is not just equivalent to but synonymous with some Boolean compound of $\varphi$, where - adapting this locution somewhat from [Smiley, 1962 ] - to be synonymous at a point in model formulas $\varphi$ and $\psi$ must not only be equivalent at that point in the sense of having the same truth-values at the point, but that must be so for arbitrary $\chi$ and $\chi^{\prime}$ differing only in that one has (zero or more) occurrences of $\varphi$ where the other has $\psi$. (This is not to say that the two formulas are synonymous, in Smiley's sense, according to the semantically specified logic here, i.e., freely interreplaceable in arbitrary contexts salva validitate. That would not be so because of the model-to-model variation as to what the relevant Boolean formula in the case of $O_{2} p$, say, would be.)

The supervenience behaviour of $O_{1}$ and $O_{2}$ is rather different. If we let $\mathcal{V}$ be the set of all valuations of the form $v_{x}^{\mathcal{M}}$ for arbitrary $\mathcal{M}=$ $\langle W, X, V\rangle$ as above and $x \in W$, then for $i$ as either 1 or 2 we do not have $p \vdash \mathcal{V}$ svc $O_{i} p$. But if we fix on a single such $\mathcal{M}$ then we do have $p \vdash \mathcal{V}$ svc $O_{i} p$ for $i=2$, though not for $i=1$, since in the latter case we can choose $x, y$ on opposite sides of the $X, W \backslash X$ boundary. (The interested reader may prefer to reformulate these $\vdash^{\text {svc }}$ observations in $\mathcal{L}_{\mathrm{D}}$ terms using the $\mathrm{D}$ connective.) Structurally we have here an analogue of the inter-world/intra-world contrast mentioned for supervenience of properties (or one-place predicates, relative to theories) in Section 8. Here, it is surfacing as inter-model vs. intra-model supervenience.

There are numerous applications, in logic and semantics, of the supervenience terminology and the idea behind it which are not under discussion here but some of this variety is worth mentioning just to close the present section. Kremer [1988] endorses a thesis of the supervenience of the extension of the predicate "is true" (in a language doing the best it can to contain its own truth predicate) on the extensions of any nonsemantic vocabulary - the supervenience of semantics, he calls it: models must not agree with respect to their interpretation of the non-semantic vocabulary (in the object language) while differing on the semantic vocabulary; a similarly inspired idea is defended in [Kremer, 2016] (a dif- 
ferent Kremer), and in this general area we also have [Bonevac, 1991]. ${ }^{24}$ Nor has there, in this area, been any shortage of talk of dependence, one famous example being [Yablo, 1982], and, for something more recent [Leitgeb, 2005]. Humberstone [2005a, p. 586] lists three supervenience conditions one might consider placing on Kripke models for modal logic, the third of which was that the truth of arbitrary formulas should supervene on the truth of atomic formulas, which is to say that any points in a model agreeing on their atomic formulas (and thus, in the propositional case, agree on the sentence letters) should agree on all formulas. And in the 'Local Supervenience Lemma' of [Humberstone, 2013, p. 705] it is shown that Kripke models for intuitionistic logic whose underlying frames are linearly ordered automatically satisfy a condition in the same ballpark: For $\mathcal{M}=\langle U, R, V\rangle$ with $R$ a reflexive linear order on $U$, and $\varphi$ is a formula in which at most the variables $q_{1}, \ldots, q_{n}$ appear, if we have $x, y \in U$ with $R x y$ and $\mathcal{M} \models_{x} q_{i}$ iff $\mathcal{M} \models_{y} q_{i}$ for all $i \in\{1, \ldots, n\}$, then $\mathcal{M} \models_{x} \varphi$ iff $\mathcal{M} \models_{y} \varphi \cdot{ }^{25}$ The other two conditions from [Humberstone, 2005a] speak of the "identity of points" being supervenient on (1) the truth of formulas, or (2) the truth of atomic formulas. This means that a model should not contain distinct points verifying precisely the same (1) formulas or (2) atomic formulas. It is one of those ideas which many people feel differently about in the temporal and the alethic modal cases, with a greater reluctance to tolerate different worlds with the same truths than different times with the same truths (eternal recurrence etc.). Such 'supervenience of identity' theses are the bread and butter of the identityof-indiscernibles literature in metaphysics: see, for example, [Khamara, 1988] for a range of classes of properties indiscernibility in respect of which might be considered to imply identity.

Understanding supervenience as preservation of agreement in this sort of way, the most important logical area in which this notion puts in an appearance is in the semantic characterization of implicit definition a (for definiteness, let's say, first order) theory $T$ whose language in-

24 Even setting aside the idea of the truth of statements about truth, one recalls the oft-cited dictum from [Bigelow, 1988, p. 132]: "truth supervenes on being" - any worlds with the same things existing in them cannot differ as to what statements are true in them.

25 The 'local supervenience' label here uses 'local' in the sense familiar from applications of Kripke semantics ('local consequence' etc.) has nothing to do with the spatially inspired sense of 'local supervenience' as this phrase has been used in the philosophy of mind (such as in [Davies, 1992]). 
cludes expressions items $E_{1}, \ldots, E_{n}, E$ as items of primitive non-logical vocabulary - predicate symbols or function symbols (including individual constants) - implicitly defines $E$ in terms of $E_{1}, \ldots, E_{n}$, when any two models of $T$ with the same universe which agree on the interpretation (i.e., the extension) of $E_{1}, \ldots, E_{n}$ agree on the interpretation of $E$. Since this relation is a closure relation we could, though typically the expressions concerned are not formulas, use 'consequence relation' notation to abbreviate this to: $E_{1}, \ldots, E_{n} \vdash \vdash_{T}^{\text {def }} E$. (Note that the agreement relation here, unlike that underlying the relation $\vdash_{\mathcal{V}}^{\text {svc }}$ for any choice of $\mathcal{V}$ - see Remark 1.7(ii) - a bipartite equivalence relation, since there are typically more than two ways to interpret a given $E_{i}$ over a given domain.) Usually such notions are introduced a special restriction to the effect that $E$ is not among $E_{1}, \ldots, E_{n}$, deliberately avoided here since this would stop $E_{1}, \ldots, E_{n} \vdash_{T}^{\text {def }} E$ being a closure relation (for which we need, most conspicuously, that for all $E$ in the vocabulary of $T$ : $\left.E \vdash \vdash_{T}^{\text {def }} E\right)$. The restriction would need to be reintroduced for purposes of stating Beth's Theorem using this apparatus in order to disallow circularity in the explicit definition thereby promised (in the present case, taking $E$ as a monadic predicate letter, for example, and thinking of $\forall x(E x \leftrightarrow E x)$ as a would-be definition. ${ }^{26}$ An issue corresponding to the requirement explicitly not imposed here (i.e., that $E \notin\left\{E_{1}, \ldots, E_{n}\right\}$ ) has also arisen in the property supervenience literature, as we shall see in Section 8 (under 'First Comment').

26 Though supervenience is not mentioned, Beth's Theorem is discussed with the related notion of reducibility in mind in [Hellman and Wilson, 1975] and [Tennant, 1985]; for other connections with definability theory, see [Humberstone, 1998a]. Supervenience and Beth's Theorem are explicitly linked in [Petrie, 1987], though the kind of supervenience concerned is one - global supervenience - roughly glossed here in Remark 8.4, arises only incidentally the more detailed discussion of supervenience in Section 8 below. Some of the supervenience theses of the preceding paragraph fall under this heading, as would a necessitated variation of David Lewis's "Humean Supervenience", which as it stands is advanced as a contingent and a posteriori thesis, and so not a supervenience thesis according to the present understanding (see [Stalnaker, 2003] for the relevant sources and discussion), though it is an analogue of claims that would be made using the conditional or hybrid three-place consequencelike relations mentioned in Remark 1.3(iv): given such-and-such assumptions, there is no difference in these respects without a difference in those. 


\section{Team Semantics and the Logic of Dependence}

We return to the logic $\mathcal{D}$ mentioned at the start of Section 1 as being introduced in $\S 2.5$ of [Goranko and Kuusisto, 2018], extracted from materials in [Väänänen, 2008], for which the primitive connectives chosen are $\wedge$ and $\vee$ with $D$ having only propositional variables in its scope and with occurrences of $\neg$ restricted to having as their scope propositional variables or D-formulas. The model-theoretic semantics for this language is described as a 'team' semantics, meaning that the truth-conditions of various compounds are given relative to sets ('teams') of points in the models rather than just relative to individual points, and in the case of the clause for $\vee$ involves quantification over such sets. ${ }^{27}$ The truth relation in this case Goranko and Kuusisto denote by "Iト" to minimize any risk of confusion with the truth relation $\models$ discussed in Section 1 above, and as with the latter case (see the discussion following the definition of $\models$ in that section), they actually take $W$ to be set of truth-value assignments to the propositional variables rather than as in conventional Kripke-style model theory. Accordingly what they write for the definition of truth is as follows; ${ }^{28}$ note that there is no connection with the use of " $\vdash$ " for generalized consequence relations in Remark 1.3(i):

○ $W \Vdash p_{i}$ iff $w\left(p_{i}\right)=T$ for all $w \in W$

$\circ W \Vdash \neg p_{i}$ iff $w\left(p_{i}\right)=F$ for all $w \in W$

$\circ W \Vdash \mathrm{D}\left(p_{1}, \ldots, p_{n} ; q\right)$ iff for all $u, v \in W$, if $u\left(p_{i}\right)=v\left(p_{i}\right)$ for each $i \leq n$, then $u(q)=v(q)$

27 The word team in the present setting is taken over from quantificational dependence logic [Väänänen, 2007a], replacing the use in [Hodges, 1997] of the word trump. While the latter did not seem especially suggestive, the former seems positively misleading, in view of Hintikka's appeal to teams of players to model informational independence in game-theoretic semantics (by constraining ccommunication between the players, e.g. in [Hintikka, 1996, p. 49]).

28 Except for the fact that we write " $p_{i}$ " for an arbitrary propositional variable rather than $p$-though $q$ figures in this way in the treatment of D - variables and $T, F$ rather than 1,0 , and we omit the subscript " $\mathcal{D}$ " on "IF", as we similarly omitted the subscript " $D$ " on " $=$ " in Section 1 above, trusting the different turnstile notation to suffice for alerting the reader as to which language and semantics are at issue. Also I have replaced the variables $U, V$ over subsets of $W$ in the clause here for $\vee$ by $X, Y$, to avoid using " $V$ " for purposes other than the kind we saw it put to in Section 1: assigning truth-sets in a Kripke-style model to sentence letters, for which it will be used again below as we reformulate the following truth-definition into a more familiar guise. 
$\circ W \Vdash \neg \mathrm{D}\left(p_{1}, \ldots, p_{n} ; q\right)$ iff $W=\varnothing$

$\circ W \Vdash \varphi \wedge \psi$ iff $W \Vdash \varphi$ and $W \Vdash \psi$

$\circ W \Vdash \varphi \vee \psi$ iff for some $X, Y \subseteq W$ such that $X \cup Y=W$ we have $X \Vdash \varphi$ and $Y \Vdash \psi$

Goranko and Kuusisto are at some pains to explain the rather surprisinglooking clause for $\neg \mathrm{D}$ formulas here, taken over from [Väänänen, 2007a], but we will not need the treatment of negated D-formulas in what follows, when we come to look at the interplay between disjunction and (unnegated) D-formulas. There is also something disturbing about the restrictive formation rules for the present language. One can see from the semantics why there are only propositional variables in the scope of D, perhaps, namely because it is only for these formulas that we have a local notion of truth explicitly visible in the definition of $\Vdash$, given by whether $v\left(p_{i}\right)=T$ or $v\left(p_{i}\right)=F$; a treatment like that provided for $\mathrm{D}\left(\varphi_{1}, \ldots, \varphi_{n} ; \psi\right)$ in the inductive definition of $\models$ in Section 1 would ask for agreement between any pair of elements of $W$ in respect of the $\varphi_{i}$ (our $\equiv_{\varphi}^{\mathcal{M}}$ relation) to imply agreement of that pair over $\psi$. One might respond that the present clause for $\mathrm{D}$ quantifies over elements of $W$ rather than subsets of $W$, but in any case these elements have their singletons, and even if one-element sets do strange things to D-formulas, as we shall recall (especially from Goranko and Kuusisto in Section 5), the rationale for excluding all but sentence letters from the scope of $D$ remains obscure for this presentation of the semantics. Remark 5.1, below, will make it clearer why, in the game-theoretic version of the semantics in Väänänen, this feature is hard to avoid. ${ }^{29}$

Another misgiving one might have about the present language and its semantics arises over the justification for restricting the occurrences of negation so that they all precede either propositional variables or D-formulas. One might say: this is all right because every formula is equivalent by De Morgan and double negation transformations to a for-

${ }^{29}$ Ciardelli [2016a] takes a less conciliatory tone: "though the dependence atom of PD is restricted to atomic sentences, there is no serious reason for this limitation." Here PD is a Väänänen-style propositional dependence logic, and dependence formulas are referred to as dependence atoms (a terminology which would indeed be appropriate in the case of quantificational dependence logic). The footnote on the page of [Ciardelli, 2016a] in question, as well as the discussion on p. 168, give valuable further information on these issues, distinguishing allowing arbitrary formulas as components of dependence formulas from allowing arbitrary formulas other than dependence formulas as components. 
mula in this restricted class. But then shouldn't the semantics tell us that the formulas in question really are equivalent to each other, which it cannot do without providing truth-conditions for the missing formulas? (This echoes a concern expressed by Smiley in [Smiley, 1996]. There are further problems about negation-normal form restrictions raised for Hintikka-Sandu style independence-friendly logic, the immediate ancestor of Väänänen's approach, raised in [Tennant, 1998, p. 96]. For the sake of simplicity we try to avoid contact with issues concerning negation here. ${ }^{30}$ )

Instead of taking up these issues, let us re-describe the team semantics in more familiar terms, as was done in Section 1 for the standard semantics. The difference will be, of course, that truth is relativized to a set of points - a "team" - rather than to a single point (in a model). Models themselves can be taken to have the same shape as in defining $\models$ in (D1.1) on p. $6,{ }^{31}$ though as a reminder of our new relata, we continue to use $\Vdash$ as our truth relation; letting $\mathcal{M}$ be $\langle W, V\rangle$ as before, we define, for arbitrary $U \subseteq W$ and formula $\varphi$ of the language of $\mathcal{D}$, the relation $\mathcal{M}, U \Vdash \varphi$ :

- $\mathcal{M}, U \Vdash p_{i}$ iff $U \subseteq V\left(p_{i}\right)$

- $\mathcal{M}, U \Vdash \neg p_{i}$ iff $U \cap V\left(p_{i}\right)=\varnothing$

- $\mathcal{M}, U \Vdash \mathrm{D}\left(p_{1}, \ldots, p_{n} ; q\right)$ iff for all $x, y \in U$, if $x \in V\left(p_{i}\right) \Leftrightarrow y \in V\left(p_{i}\right)$ for all $i \leq n$, then $x \in V(q) \Leftrightarrow y \in V(q)$

- $\mathcal{M}, U \Vdash \neg \mathrm{D}\left(p_{1}, \ldots, p_{n} ; q\right)$ iff $U=\varnothing$

- $\mathcal{M}, U \Vdash \varphi \wedge \psi$ iff $\mathcal{M}, U \Vdash \varphi$ and $\mathcal{M}, U \Vdash \psi$

30 Tennant also addresses the question of the emergence of a second "Boolean" negation alongside the usual negation game-theoretically explained in terms of changing verifier-falsifier roles, in what Hintikka calls extended IF logic in [Hintikka, 1996], with a correspondence extension of Väänänen's dependence logic called by him team logic, in Chapter 8 of [Väänänen, 2007a] - or see [Väänänen, 2007b]. As Väänänen remarks, concerning this negation, which he writes as " " as follows on p. 144 of [Väänänen, 2007a]: "The introduction of $\sim$ unfortunately ruins the basic gametheoretic intuition," somewhat reminding one of the frosty reception of Boolean negation in some sections of the relevant and paraconsistent communities - cf. Chapter 5 of [Priest, 2006]. The behaviour of the favoured negation in dependence logic is not just non-classical, since neither player need have a winning strategy in the game based on a given formula and model, but rather more seriously anomalous than this: see [Burgess, 2003], and for some technical follow-up, [Kontinen and Väänänen, 2011].

31 In particular we can require, as there, that the universe $W$ of the models is non-empty, while allowing the $U$-parameter over subsets in the following definition to take $\varnothing$ as a value. 
- $\mathcal{M}, U \Vdash \varphi \vee \psi$ iff for some $X, Y \subseteq W$ such that $X \cup Y=U$ we have $\mathcal{M}, X \Vdash \varphi$ and $\mathcal{M}, Y \Vdash \psi$

As in Section 1, we can define a consequence relation in terms of this apparatus, and again we use the same turnstile for that purpose: ${ }^{32}$

(D3.1) $\varphi_{1}, \ldots, \varphi_{n} \Vdash \psi$ iff for every model $\mathcal{M}=\langle W, V\rangle$ and all $U \subseteq W$, if $\mathcal{M}, U \Vdash \varphi_{i}$ for each $i \leq n$, then $\mathcal{M}, U \Vdash \psi$.

Proposition 2.3 of [Goranko and Kuusisto, 2018] then says that if $\varphi$ is a D-free formula in negation normal form (i.e., all occurrence of $\neg$ being directly applied to propositional variables), then for any model $\mathcal{M}=\langle W, V\rangle$, we have, using the revised account of $\Vdash$ just given (and that of $\models$ from Section 1$)$ :

$$
\mathcal{M}, W \Vdash \varphi \text { iff } \mathcal{M}, w \models \varphi \text { for all } w \in W .
$$

They remark:

This proposition shows that team semantics simply lifts the semantics of propositional logic (in negation normal form) from the level of individual assignments onto the level of sets of assignments. Thus, team semantics can be used in scenarios where assertions (encoded by formulae of propositional logic) are made about sets of possible worlds, and the intention of the assertions is to claim that any world in the set satisfies the formula.

Something the team semantics also suggests is the option of rejecting assertions about individual worlds on the grounds that there are no such worlds (at least, other than the actual world), which would mean that the teams themselves could no longer be thought of as sets of worlds but perhaps conceived more neutrally as regions of logical space, as it is put in [Humberstone, 1981], between which there are quasi-mereological part/whole relations and the non-empty regions could be thought of as potentially refinable (or further specifiable) possibilities. This line of thought, and the greater generality it promises, have recently been pursued extensively in work by Wesley Holliday, whose [Holliday, 2016] explicitly mentions the team semantics approach as a special case; see also [Holliday, 2014]. On this neutral approach one allows for the possibilities to be sui generis, as in the motivating "there are no such entities

32 Reminder: Remark 1.3(i) mentions the unrelated uses of this same notation in the present paper as well as earlier discussions. 
as fully specific possible worlds" idea, as well as allowing for them to be construed as sets of points with the part-whole relation in that case amounting to class inclusion. On the non-set-theoretic realisation the " $\cup$ " symbol appearing in the $\Vdash$-clause for $\vee$ would represent something closer to mereological fusion.

\section{Disjunction and Partiality}

The reference to partiality in the title of the present section is to the partial or 'gappy' nature of the semantic approach we have just been discussing. Because a region is not just a point (or unit set thereof) a statement may fail to be true of a whole region (being false of some subregions) while at the same time failing to be false of that region (being true of some subregions). Here, we are interested in the semantic heterogeneity of regions as it bears on disjunction rather than negation, and will accordingly make no further play with the idea of falsity in the informal remark just made; any further reference to falsity may be interpreted simply as indicating absence of truth. Returning, then, to disjunction itself, what follows is the explanation of the key idea as it appears in [Humberstone, 1988a, p. 64f.] which starts from Urquhart's motivating idea in his semantics for relevant logic, namely to think of the entities to which truth is relativized in one of his semilattice models as representing bodies of information. In this quotation, schematic letters $A, B$, are replaced by the $\varphi, \psi$, of the present discussion and the original use of " $=$ " is replaced with "\|I" to avoid clashes with Goranko and $\mathrm{Ku}$ usisto's contrast between " $=$ " and "IF" for the truth relations associated respectively with $\mathcal{L}_{\mathrm{D}}$ and $\mathcal{D}$ :

Indeed, a piece of information may be regarded as a possibility in epistemic dress: all information is information that you are in such-andsuch a region of logical space. And, in contrast with the case of possible worlds, a disjunctive statement may hold throughout a region, without this being so for either disjunct, for the same reason that a barnyard can be full of things each of which is a sheep or a goat, without being full of sheep or full of goats. In the latter case this can only be so if the yard can be divided without remainder into a (perhaps spatially scattered) part which is full of sheep and a part which is full of goats. Similarly, we are led to require that a $(\varphi \vee \psi)$-verifying region should be exhaustively composed out of a $\varphi$-verifying and a $\psi$-verifying subregion. We denote this mode of composition by "+". The subregions may in 
general overlap, of course, should the disjuncts be compatible. ${ }^{33}$ Some will want to think of regions of logical space as sets of possible worlds, and think of "+" in this context as representing set union. In fact, though $(\ldots)$, there is no need to do so, and we may prefer to think more mereologically, with $x+y$ as the smallest whole of which both $x$ and $y$ are parts. The difference between these two views does not matter for what follows, however. Either way, what is suggested is the following clause for disjunction in the definition of truth, once we have incorporated the + operation into our models:

$$
\begin{gathered}
(*) x \| \varphi \varphi \vee \psi \text { iff there exist } y, z \text { such that } x=y+z \text { and } \\
\qquad y \| \varphi \text { and } z \| \Vdash \psi .
\end{gathered}
$$

One might think it necessary to add an extra disjunct to the righthand side here in case for one of the disjuncts there is no subregion of $x$ verifying that disjunct. ${ }^{34}$ But this is not needed if we admit the empty region as a subregion of every region - a unique impossibility nestled among the possibilities. As, in general the smaller the region the more is true throughout that region, the empty region - call it $0-$ should verify all formulas, and as the notation suggests, this serves as a (two-sided) identity/neutral element for the + operation, the reduct $\langle W,+\rangle$ being presumed to be a semilattice; a further condition will be imposed presently. (In the specifically set-theoretical team semantics 0 is $\varnothing$, and as we have already seen, + is $\cup$.)

Simplifying the setting of this idea as it appears in [Humberstone, 1988a] by omitting Urquhart's binary operation (for dealing with the relevant implication connective), this gives us models $\langle W,+, 0, V\rangle$ for the language with $\vee$ when $V$ is as in the following paragraph, before which we need one further condition on the frames $\langle W,+, 0\rangle$ of these

33 The subregion or 'part of' relation implicit in the discussion can be taken as the relation $\leq$ defined below. Overlapping here is understood as having a non-empty common part. The empty region 0 will be introduced below.

34 Thus one would add "or $x \| \vdash \varphi$ or $x \| \vdash \psi$ "; this is what happens in Definition 11 of [Došen, 1989]. (Kit Fine made a similar suggestion in the discussion after [Humberstone, 1988a] was presented at a conference at ANU in February 1985, as an alternative to the treatment using 0 . What would happen if we left out 0 and did not put in this addendum? See note 44 and the last paragraph of Section 5.) Thus the 1980s saw quite an interest in these 'conjunctive' style treatments of disjunction. Perhaps the first of these to appear in print were those of [Orłowska, 1985, p. 455] and then [Komori, 1986, (3) on p. 11]. Further references to similar work in this period and later can be found in the final 'Notes and References' paragraph on p. 924 of [Humberstone, 2011]. 
models, called in [Humberstone, 1988a] (where the frames are actually written as $\langle S,+, 0\rangle)$ Decomposition, and formulated with the aid of the partial ordering $\leq$ (defined as usual by: $x \leq y \Leftrightarrow x+y=y)$ :

For all $x, u, v \in W$ if $x \leq u+v$ then there exist $u^{\prime} \leq u, v^{\prime} \leq v$ such that $u^{\prime}+v^{\prime}=x$.

Satisfying this decomposition condition makes the semilattice $\langle W,+\rangle$ into what is generally called a distributive (join-)semilattice. Note that in the special case of the team semantics above, with + as $\cup$ (and $\leq$ as $\subseteq$ ), this condition is satisfied (taking $u^{\prime}, v^{\prime}$ respectively as $u \cap x, v \cap x$ ).

Given a frame $\langle W,+, 0\rangle$ as here described, a map $V$ assigning subsets of $W$ to the propositional variables yields a model on the frame provided two conditions are satisfied for each $p_{i}$ :

- The + condition: $x+y \in V\left(p_{i}\right)$ iff $x \in V\left(p_{i}\right)$ and $y \in V\left(p_{i}\right)$ (all, $x, y \in W)$.

- The 0 condition: $0 \in V\left(p_{i}\right)$.

These conditions are then seen [Humberstone, 1988a, p. 68f.] to spread to arbitrary formulas, in the sense that an induction on formula complexity yields the following two lemmas:

Lemma 4.1 (Plus Lemma). For any model $\mathcal{M}=\langle W,+, 0, V\rangle$, for all $x, y \in W$ and any formula $\varphi: \mathcal{M}, x+y \| \vdash$ iff $\mathcal{M}, x \| \vdash$ and $\mathcal{M}, y \| \vdash$.

Lemma 4.2 (Zero Lemma). For any model $\mathcal{M}=\langle W,+, 0, V\rangle$ and any formula $\varphi$, we have $\mathcal{M}, 0 \Vdash \varphi$.

This gives disjunction its classically (or intuitionistically) expected properties; for whose formulation we use (as in (D1.1) on p. 6 and (D3.1) on p. 37) the truth-relation notation to double as a notation for a consequence relation: $\Gamma \| \Vdash \psi$ iff for any model $\mathcal{M}$ of the kind currently in play, and any $\mathcal{M}$ element $x$, whenever $\mathcal{M}, x \| \vdash$ for each $\varphi \in \Gamma$, then $\mathcal{M}, x \| \vdash \psi$ :

Proposition 4.3. For all formulas $\varphi, \psi, \chi$ and sets of formulas $\Gamma$ :

(i) $\varphi \| \vdash \varphi \vee \psi$ and $\psi \| \vdash \varphi \vee \psi$;

(ii) If $\Gamma, \varphi \| \Vdash \chi$ and $\Gamma, \psi \| \Vdash \chi$ then $\Gamma, \varphi \vee \psi \| \vdash \chi$.

Proof. For (i), use the Zero Lemma, and for (ii), the Plus Lemma. (Further details: see [Humberstone, 1988a].) 
Remark 4.4. If the sole connective involved is $\vee$, the special case of condition (ii) of Prop. 4.3 in which $\Gamma=\varnothing$ suffices for the general case, and for this only the "if" (or "upward"), direction of the Plus Lemma is required, though the general form with arbitrary $\Gamma$ is essential when other connectives (such as $\wedge$ ) are present, and for this both direction of the Plus Lemma are called for.

It is well known that in the language with $\vee$ as its sole connective, the syntactic conditions given in Proposition 4.3 (along with the defining conditions for consequence relations in general) suffice for completeness - and indeed, as Remark 4.4 recalls, some weakening of the second condition is possible in this case. If we include also $\wedge$ in our language (understood in the usual way, as in the $\Vdash$-truth-definition given earlier) then along the familiar principles $(\varphi \wedge \psi\|\Vdash \varphi ; \varphi \wedge \psi\| \vdash \varphi ; \varphi, \psi \| \vdash \varphi \wedge \psi)$ give a similarly sound and complete logic for the conjunction-disjunction fragment of classical logic. Most importantly, the Plus Lemma continues to hold. Negation is not discussed in [Humberstone, 1988a], or in the later summary in [Humberstone, 2011], though relevant implicationnot to the point here - is. Section 5 looks at the simultaneous presence of the $\mathrm{D}$ connective (or the $\mathrm{D}_{n}$, if the multigrade $\mathrm{D}$ is eschewed). The talk of possibilities and regions as opposed to points notwithstanding, the "+" treatment of disjunction is not that provided in [Humberstone, 1981], and one may wonder about how the treatment of $\vee$ there (which piggy-backs on construing $\varphi \vee \psi$ as $\neg(\neg \varphi \wedge \neg \psi)$ with $\neg$ given an intuitionistic-looking treatment) is related to that provided by the 'plus' semantics. Though we do not go into the matter further here, the answer to this question can be found in Lemma 8.4 of [Holliday, 2016] which provides conditions on possibility frames sufficing for the two interpretations of $\vee$ to be equivalent.

While we have the Plus Lemma before us, let us pause to observe that, in the terminology of [Humberstone, 2011], it tells us that the operation + forms conjunctive combinations on the left of the relational connection $(R, W, F)$ where $W$ is the universe of one of our models $\mathcal{M}=$ $\langle W,+, 0\rangle, F$ is the set of formulas of the language under consideration and $R \subseteq W \times F$ is the relation holding between $x \in W$ and $\varphi \in F$ when $\mathcal{M}, x \| \vdash$. According to Theorem 0.14.2 of [Humberstone, 2011], if a relational connection provides conjunctive combinations on the left and also disjunctive combinations on the right (or indeed vice versa), then it satisfies the Cross-Over Condition, which here amounts to this:

If $\mathcal{M}, x \| \vdash \varphi$ and $\mathcal{M}, y \| \vdash \psi$, then either $\mathcal{M}, x \| \vdash \psi$ or $\mathcal{M}, y \| \vdash \varphi$. 
This is clearly not a condition that we would want our models in general to satisfy, so we had better make sure that our semantic apparatus does not also provide for disjunctive combinations on the right (i.e., on the $F$-as opposed to the $W$-side of the relational connection). This is what happens in [Väänänen, 2008, §8]. In the passage here quoted, Väänänen uses his notation " $=\left(p_{1}, \ldots, p_{n}, q\right)$ " in place of the " $\mathrm{D}\left(p_{1}, \ldots, p_{n} ; q\right)$ " notation of Goranko and Kuusisto (in their exposition of $\mathcal{D}$ ), with $=(q)$ for the $n=0$ case (which we have been representing as $\mathrm{D} q$, and Goranko and Kuusisto write as $\mathrm{C} q$ ). As foreshadowed in the $n=1$ case in Remark 4.4 above, Väänänen begins by noting that we can define $=\left(p_{1}, \ldots, p_{n}, q\right)$ in terms of $=(q)$ as the disjunction of the formulas $\varphi_{i} \wedge=(q)$, where the $\varphi_{i}$ are the $2^{n}$ conjunctions each of the conjuncts of which is either $p_{j}$ or $\neg p_{j}(1 \leq j \leq n)$. The reference to disjunction there was to $\vee$ as treated by the team semantics (+-style, with $\cup$ ). He then goes on to entertain adding a further disjunction connective:

We can define $=(p)$ if we add to our modal dependence language a Boolean disjunction $\varphi \vee_{\mathbf{B}} \psi$ with the obvious meaning that $\varphi \vee_{\mathbf{B}} \psi$ is true in a set iff $\varphi$ is true in the set or $\psi$ is. ${ }^{35}$

This would-be Boolean disjunction (unlike $\vee$ ) forms disjunctive combinations on the right, and accordingly cannot be expected to cohabit peacefully with $\vee$ in the case in which the Plus Lemma holds generally, for the reason already given. And it is clear that this lemma is already compromised by the presence of $\vee_{\mathbf{B}}$. In the team semantics approach, this amounts to a failure of compounds formed by this connective being what Väänänen (adapting a usage of Hodges') calls flat formulas: formulas true w.r.t. $X \subseteq W$ iff true over each $\{x\}$ for which $x \in X$, as is recognised in [Hella et al., 2014], where $\varphi \vee_{\mathbf{B}} \psi$ is written as $\varphi \otimes \psi$ and such compounds are excluded - along with dependence formulas - from a language all formulas of which are noted to be flat: the language of negation normal form formulas with $\wedge, \vee, \square$ and $\diamond$. (When presenting his version, 'team logic' - see note 30 above - of Hintikka's extended IF logic, Väänänen changes his notation so that $\vee$ is now the Boolean disjunction and the disjunction originally represented by this symbol is now

35 The sentence continues with a clause giving the conditions under which $\neg\left(\varphi \vee_{\mathbf{B}}\right.$ $\psi$ ) is true relative to a set of points, which need not concern us. In quoting this passage, from p. 253 of [Väänänen, 2008], I have replaced Väänänen's schematic letters $A, B$ with $\varphi, \psi$ for conformity with the rest of our discussion (which follows [Goranko and Kuusisto, 2018] in this respect). 
written as $\otimes$. It is defined De Morgan style using the Boolean negation mentioned in note $30 .{ }^{36}$ )

Groenendijk [2009] writes what is essentially Väänänen's $\vee_{\mathbf{B}}$ as $\vee$ and takes it as the fundamental disjunction connective, emphasizing the fact that a disjunction formed with its aid can be true w.r.t. $\{w\}$ and w.r.t. $\{v\}$ without being true w.r.t. $\{w, v\}$, regarding this as the defining characteristic of inquisitive formulas which raise issues or questions rather than settling them. ${ }^{37}$ Ciardelli in $[2016 \mathrm{a} ; 2016 \mathrm{~b}]$ - though not in

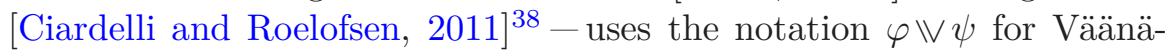
nen's $\varphi \vee_{\mathbf{B}} \psi$, calling the connective concerned inquisitive disjunction. (It is called ) We will look at some aspects of the behaviour of this and other connectives in the setting of inquisitive logic in Section 6, and here offer one suggestion as to something evidently puzzling Ciardelli at p. 235 of [2016a], when he cites Ebbing et al. [2013] as discussing inquisitive disjunction and writes parenthetically "which they refer to, curiously, as classical disjunction". The suggestion is that the usual presumption or convention that the metalogic for discussions of various ('object') logics is to be classical logic, so whenever the clause governing compounds formed by a particular in the inductive definition of a relation

${ }^{36}$ Is the use of the tensor notation familiar from linear logic coincidental here? Abramsky and Väänänen [2009] use this notation and explicitly say (p. 208) disjunction, as treated by the Hodges semantics, "is actually multiplicative conjunction"a surprising claim in view of Proposition 4.3(i) above. At the end of the following section, we note the similarity, at a suitable level of abstraction, between the present treatment of disjunction and a plausible treatment of temporal conjunction ("and then"). Though citing [Abramsky and Väänänen, 2009], [Ciardelli, 2016a, p. 160, inc. note 2] rather more plausibly describes $\otimes$ as representing tensor disjunction.

37 According to the presentation of 'inquisitive semantics' in [Groenendijk, 2009], this point about the favoured disjunction is put rather differently, with all formulas evaluated w.r.t. ordered pairs of worlds: such a disjunction can be true w.r.t. $(w, w)$ and w.r.t. $(v, v)$ without being true w.r.t. $(w, v)$; but note 7 in [Groenendijk, 2009] explains that the order is immaterial and the whole thing can be done w.r.t. arbitrarily large sets of worlds. We presume this generalization in what follows. The history of the evolution of the subject from the 'pairs' version to the general version is explained in the middle paragraph of [Ciardelli, 2016b]; see also [Ciardelli et al., 2015a].

38 Or in Ciardelli [2009], in both of which the notation $\vee$ is used, and with good reason, since one of the aims of these works is to sketch inquisitive logic as something like an intermediate logic, for which purpose one needs to align inquisitive disjunction with the usual fundamental disjunction connective of intuitionistic logic. ('Something like' an intermediate logic, because as usually understood intermediate logics - indeed often logics in general - are understood as needing to be closed under Uniform Substitution, which we do not have here. See further Section 6 below.) 
in the 'satisfies', 'verifies',... family is given using "or", that connective is referred to as classical (or indeed, as with Väänänen, Boolean) disjunction. What happens in the present case is that there are two salient semantic relations in this family in play: we have the relation of truth at a world, and truth w.r.t. a set of worlds. The latter Ciardelli calls being supported by an information state, regarding the former as the special case in which the sets are singletons. The non-inquisitive disjunction, just called disjunction and symbolized by $\vee$ in our earlier discussion, has a "true at a world" semantic clause using "or" - what would often be called a homophonic treatment, that is - whereas relative to an information state it does not, being given a heterophonic treatment in much the same way as the team semantical plus-treatment reviewed above. On the other hand, at the level of information states it is inquisitive disjunction has gets the homophonic treatment. Thus one's tendency to call this or that classical/Boolean disjunction is conditioned by which satisfaction relation one is focussing on. ${ }^{39}$ Väänänen's $\varphi \vee_{\mathbf{B}} \psi$ (or Ciardelli's $\mathbb{V}$ ) is called blind disjunction in [Galliani, 2013b] (and perhaps first in [Japaridze, 2009]).

\section{Disjunction and Dependence}

Goranko and Kuusisto see as at least one major advantage of $\mathcal{L}_{\mathrm{D}}$ over $\mathcal{D}$ the anomalous behaviour of disjunction (the $\vee$ of Sections 1 and 2, that is) in the latter logic. The present section tentatively endorses and elaborates on this sentiment - though the approach of inquisitive logic, reviewed in Section 6 threatens to give Goranko and Kuusisto's approach a run for its money, and to reduce the appearance of an anomaly by taking a radically different stance on the internal structure of dependency claims (which have no corresponding internal structure to any approach with a primitive D operator). Here is how Goranko and Kuusisto summarise the dialectic [Goranko and Kuusisto, 2018, p. 16]:

Our argument will proceed as follows. We first argue that the semantics of formulae of the type $p \vee q$ is a good match with natural language

39 A complication: at the level of worlds, inquisitive disjunction ends up with a homophonic treatment. Note that this is only possible because of the failure of flatness - specifically of the implication: if true w.r.t. $\{w\}$ for all $w \in X$, then true w.r.t $X$-already remarked on, In Ciardelli's work on inquisitive semantics, flat formulas are called truth-conditional formulas. 
intuitions in both logics $\mathcal{L}_{\mathrm{D}}$ and $\mathcal{D}$. We then turn to examples concerning formulae of the type $\mathrm{D}(p ; q)$, and again argue that the semantics of both logics is reasonable. (In this context we also briefly discuss more complex formulae of the type $\mathrm{D}(p, q ; r)$, but this is not crucial from the point of view of our discussion.) We then argue that, despite both $p \vee q$ and $\mathrm{D}(p ; q)$ having a reasonable semantics in $\mathcal{D}$, formulae $\mathrm{D}(p ; q) \vee \mathrm{D}\left(p^{\prime} ; q^{\prime}\right)$, which combine $\vee$ and $\mathrm{D}$, are problematic. In fact, we show this even for the formula $\mathrm{D}(p ; q) \vee \mathrm{D}(p ; q)$, where both disjuncts are the same. We then continue by arguing that $\mathcal{L}_{\mathrm{D}}$, in turn, gives natural interpretations for these problematic examples.

As is observed in [Goranko and Kuusisto, 2018], every formula

$$
\mathrm{D}\left(p_{1}, \ldots, p_{k} ; q\right) \vee \mathrm{D}\left(r_{1}, \ldots, r_{n} ; q\right)
$$

is valid according to the semantics of $\mathcal{D}$; in our terminology, each such formula is a $\Vdash$-consequence ( $\Vdash$ as defined in (D3.1) on p. 37) of $\varnothing$. The reason is as follows (here reformulated to match the presentation above). Given a model $\mathcal{M}=\langle W, V\rangle$ take any $U \subseteq W$, and let $U_{q}$ and $U_{\bar{q}}$ be $V(q) \cap U$ and $U \backslash(V(q) \cap U)$ respectively. Since $U=U_{q} \cup U_{\bar{q}}$ and $q$ 's truth-value is constant over each term of this union, each of them verifies any D-compound whose final component is $q$ and so, being a union of such sets, $U$ verifies the disjunction of these compounds in $\mathcal{M}$. The extreme case is that in which there are no other components: any 'team' will verify $\mathrm{D} q \vee \mathrm{D} q$ because it can be decomposed into its $q$-verifying part and its $q$-falsifying part, within each of which $q$ 's truth-value is constant.

A variation on this observation appeared in [Väänänen, 2008, p. 244], from which Goranko and Kuusisto's dependence logic $\mathcal{D}$ is derived by removal of the (alethic) modal elements. Though there are clauses in the inductive definition of truth in [Väänänen, 2008] for both $\square$ and $\diamond$, it is the former that concerns us here. Expanding our models $\mathcal{M}$ from the definition of $\mathcal{M}, U \Vdash \varphi$ in Section 3 so as to include an accessibility relation $R \subseteq W \times W$, and writing $R(x)$ for $\{y \in W \mid R x y\}$ and $R(X)$ for $\bigcup_{x \in X} R(x)$ the relevant clause reads:

- $\mathcal{M}, U \Vdash \square \varphi$ iff $\mathcal{M}, R(U) \Vdash \varphi$.

This gives the connection between constancy and noncontingency touched on in Section 1 above: if we take $U=\{w\}$ then $\square \mathrm{D} q$ says that the truth-value of $q$ is constant over $R(w)$, i.e., $q$ is noncontingent 
at $w \cdot{ }^{40}$ But returning to the issue about disjunction, Väänänen [2008, p. 244] notes that $\square(\mathrm{D} q \vee \mathrm{D} q)$ is true w.r.t. any team (in any model) while $\square \mathrm{D} q$ need not be. ${ }^{41}$ But, unlike Goranko and Kuusisto in the purified version without the embedding under $\square$, Väänänen says nothing by way of acknowledgment that this is an unsatisfactory outcome, as it prima facie seems to be. Any sign of a failure of idempotence on the part of a would-be representation of (inclusive) disjunctive is a sign that it is time to re-think. The re-thinking urged in [Goranko and Kuusisto, 2018] consists in moving from $\mathcal{D}$ to $\mathcal{L}_{\mathrm{D}}$, in which all the evaluations are point-relative rather than set-relative and $\varphi \vee \psi$ is true iff either $\varphi$ is true or else $\psi$ is. Certainly the problem then goes away, while we still have a suitable treatment for $\mathrm{D}$-formulas, although a residual question - not explored here - arises as to what a 'possibilities as primitive' style treatment might look like for this language. (Another reaction, that embodied in inquisitive logic, involves reconceptualising D-formulas as embedding not statement-representing formulas but question-representing formulas, forcing a reconsideration of how "or" in such contexts is best interpreted. We turn to this in the following section.)

Specifically, the direction in which the idempotence equivalence is at risk of failing for disjunction when $\varphi$ is a D-formula is:

$$
\varphi \vee \varphi \Vdash \varphi
$$

Now this condition on $\Vdash$ (as holding for all $\varphi$ ) evidently follows by appeal to condition (ii) of Proposition 4.3 above, taking $\varphi=\psi=\chi$ and $\Gamma=\varnothing$, and in view of the choice of $\Gamma$, by Remark 4.4 needs only the upward direction of the Plus Lemma: what is true at $x$ and $y$ in a model is true at $x+y$. In the team semantics this amounts to the obvious point that a D-compound true w.r.t. $X$ and to $Y$ is not guaranteed, as the GorankoKuusisto examples illustrate, to be true w.r.t. $X \cup Y$. This failure of idempotence - or more precisely the analogous failure arising within the scope of an occurrence of $\square-$ is noted in [Väänänen, 2008, p. 243f.]

${ }^{40}$ For this or any non-empty choice of $U$, when $R$ is universal, the r.h.s. of the above clause reduces to: $\mathcal{M}, W \Vdash \varphi$.

41 Väänänen's preferred semantics, given before the model-theoretic version, is the game semantics, in which $\mathrm{D} q$ (or $=(q)$, as he writes it) is given a treatment making explicit reference to uniform strategies: see Remark 5.1 below. There are numerous alternatives to and developments of Väänänen's modal dependence logic, it should be noted - see for example [Galliani, 2013a; Tulenheimo and Sevenster, 2007] - though these will not be discussed here. 
of which is described a case showing that $\square \mathrm{D} p$ is not a consequence of $\square(\mathrm{D} p \vee \mathrm{D} p)$, though, as already remarked, it is there matter-of-factly presented as an illustration of the semantics rather than as something to be regretted or corrected. The following remark may be omitted by those not interested in the game-theoretic semantics we are mostly avoiding here.

Remark 5.1. Väänänen gives the game-theoretic semantics a kind of conceptual priority in [2007a; 2008], though he does also detail the Hodgesinspired compositional semantics. On the former, truth in a model is defined as possession by the verifying player of a uniform winning strategy, where uniformity is explained in terms of making the same move at the corresponding point in any play of the game. More precisely [closely paraphrasing Väänänen, 2008, p. 240] this is a strategy for the formula $\varphi$ and player (I or II) dictating, for in any two plays relative to points $c, w^{\prime}$ in the model, at which that it is that player's turn and the same token-subformula $\mathrm{D}\left(p_{1}, \ldots, p_{n} ; q\right)$ of $\varphi$ is involved, if $w$ and $w^{\prime}$ agree on the truth-values of $p_{1}, \ldots, p_{n}$ then $w$ and $w^{\prime}$ must agree on the truth-value of $q$. (Thus there is a non-local - that is 'single play' transcending - aspect to uniformity, somewhat reminiscent in this respect of the 'subgames' theme in [Hintikka and Carlson, 1979] and in Chapter 3 of [Hintikka and Kulas, 1983]; in $§ 5$ of [2008], Väänänen, presents a variant game semantics which does not invoke the concept of uniformity.) One can see how it is natural in this setting to require the dependence formulas to be compounded from sentence letters; this account certainly be complicated if Boolean complexity were allowed at this stage, and perhaps unmanageably so if dependence compounds themselves could also be among the components. Let us return to the specific case of current interest, from p. 243f. of [Väänänen, 2008], which is described a case showing $\square \mathrm{D} p$ not to be a consequence of $\square(\mathrm{D} p \vee \mathrm{D} p)$. The explanation of why the verifying player ('Player II', as Väänänen puts it) has a uniform winning strategy in a particular (pointed) Kripke model described there, in which one successor node has $p$ true at it and the other, $\neg p$, is a little cryptic. Concerning the formula $\square(\mathrm{D} p \vee \mathrm{D} p)$, or as he writes it, $\square(=(p) \vee=(p))$, Väänänen remarks: "Now player II has a uniform winning strategy: If I plays the node with $p$, she plays the left disjunct, and otherwise the right disjunct." The puzzlement this may induce is eased somewhat by saying that an equally good uniform winning strategy would be the reverse: If I plays the node with $p$, she plays the right disjunct, and otherwise the left disjunct. What would 
not work would be to choose the left - or equally the right - disjunct regardless. This would fall foul of the uniformity condition. It should be mentioned that, as already noted, after running through the gametheoretic semantics in detail, Väänänen goes on to sketch the compositional (or recursive) semantics. But the while the former operates only by evaluating formulas at points in a model, the latter relativizes truth to sets ('teams'), as in the $\Vdash$ semantics of Section 3 (though with present and interpreted as in the present section). If I am not mistaken, this means that in preferred game-theoretic incarnation of the logic, $=(p)$ is a consequence of $=(p) \vee=(p)$ (and conversely), even though $\square=(p)$ is not a consequence of $\square(=(p) \vee=(p))$ - a rather surprising departure from the monotone behaviour one expects of a $\square$ operator - a violation indeed of congruentiality (replacement of equivalents) for such an operator; we return to these themes in connection with inquisitive logic in Section 7. Note that monoton(icit)y in the present sense has nothing to do with monotonicity as a condition on consequence or generalized consequence relations Remark 1.3(iii). (For the record, the Boolean and modal primitives chosen by Väänänen are $\vee, \neg$ and $\diamond$, since these make for a very elegant presentation of the game-theoretical semantics.) $\triangleleft$

The same matter-of-fact tone characterizes the presentation of several examples in quantificational rather than modal dependence logic touched on in [Väänänen, 2007a], including one (Example 5.9, p. 75) adapted from [Janssen, 2002]. And for the present logic we saw already in note 13 the equivalence of $\mathrm{D}(p ; q)$ with $(p \wedge \mathrm{D} q) \vee(\neg p \wedge \mathrm{D} q)$ (suppressing here the numerical superscripts appearing there), each disjunct of which has $\mathrm{Dq}$ as a consequence - so if all had been well with $\vee$-elimination the whole disjunction would have had that consequence, while as this stand all we are entitled to conclude is $\mathrm{D} q \vee \mathrm{D} q$. In terms of the plus semantics, what we need here is the upward direction of the Plus Lemma, which would not hold for D-formulas.

The Plus Lemma amounts to a universal declaration of flatness, but this is evidently not something one would want if there are formulas whose rationale is to record heterogeneity, which should not be expected to percolate downward, or whose rationale is to record homogeneity, which should not be expected to percolate upward. The latter is what is relevant to case of D-compounds and will come to the fore in the following section (on inquisitive semantics), in which the upward and downward directions are referred to as regularity and persistence, and the is latter 
retained while the former is abandoned for formulas which are intended to represent questions rather than questions, and this feature is rather more fully exploited than in the case of Väänänen's dependence logic (without the connective $\vee_{\mathbf{B}}$ mentioned toward the end of the preceding section). But for the remainder of the present section, we give the upward and downward directions equal time.

It is helpful to step back from the modal interpretation and turn to the temporal interpretation. You could spend an afternoon alternating between swimming and sunbathing. This does not mean that every subinterval of the afternoon, let alone every unit subinterval (consisting of one instant) was spent alternating between these two activities. Or we could have a spatial example: even setting to one side regions - pointsized or larger - too small to have a colour, so that we can agree that every part of a red surface is red, we would not be prepared to agree that every part of a polka-dotted surface is polka-dotted. Nelson Goodman $[1977$, p. 38f] called predicates guaranteed to be true of all parts of an object, whenever they were true of the object, dissective, and predicates which were true of the mereological sum (or fusion) of any two or more objects they were true of collective. These are essentially the downward and upward directions of the Plus Lemma in which the objects are the elements of the models and the predicates are those of verifying this or that formula (though nominalistic scruples tended to exile anything corresponding to the 0 of our models). ${ }^{42}$

The reference to homogeneity here - which is essentially the flatness property mentioned in the preceding section for the case in which there are spatial points or temporal instants making up the regions or intervals under consideration (and otherwise just means the similarity in the respect in question of a whole to all of its parts) - is an echo of a usage of this term in Chapter 3 of [Taylor, 1985], in which he elaborates on some preliminary remarks on Aristotle's distinction between Stasis, Energeia and Kinesis verbs (pp. 58-64) with a subdivision of E(nergeia)-verbs into the homogeneous and heterogeneous E-verbs. The homogeneous cases are those whose application to a subject w.r.t. an interval implies their application (to that subject) within any subinterval - where unit sets of

42 The 'collective' terminology was unfortunate because what is usually called collective predication does not involve what Goodman called collective predicates. For example one has the collective reading of "The red book and the green book weigh one kg." indicated by inserting "together" before "weigh", though "weighs one kg." is anything but a collective predicate in Goodman's sense. 
instants do not count as intervals ("periods," Taylor actually writes). They include (p.71) moves, ponders, and blushes according to Taylor, whereas heterogeneous E-verbs are those not similarly temporally dissective and include chuckles, giggles and talks; Taylor is also concerned with an analogy between this contrast and that between homogeneous (e.g. gold - at least as conceived before atomic theory) and heterogeneous (e.g., fruit cake) kinds of 'stuff'. It is not clear why we are talking about the stuff itself in the latter case but only about the verbs in the former case: compare the title of a well-known chapter of [Kenny, 1963], "States, Activities, and Performances", though, admittedly, Kenny's criteria explicitly take a linguistic turn with tests concerning static (one would now say stative) verbs, performance (= kinesis) verbs, and activity (= energeia) verbs. It should be noted that for considerations of aspect (in the linguistic sense) it is not actually verbs but verb phrases that tests in question apply to. ${ }^{43}$ Indeed the case of moves, already mentioned as one of Taylor's homogeneous E-verbs, illustrates this since moves one metre is not true of an object w.r.t. arbitrary subintervals of periods w.r.t. which it is true.

Contraposing the last example, one gets a response to one version of Zeno's arrow paradox. (Zeno of Elea this time, not the Zeno Vendler mentioned in note 43.) Call an arrow relatively stationary over a period if it no part of it changes position by more than one metre over the period. In this - perhaps one might say cheap version (the genuine article requiring a consideration of durationless instants) - of the paradox, the issue would be put: take any subinterval of the period of an arrow's flight across a field short enough that the arrow is relatively stationary over that period. Since the whole period of its flight can be decomposed into such periods over which it is relatively stationary, the arrow must be relatively stationary over the whole period and therefore cannot cross the field. The obvious reply would be that being relatively stationary is not a property inherited upward from periods to more extensive periods of which they are subintervals. In the modal case a claim of noncontingency over a region of logical space is similarly a claim of homogeneity (no difference in truth-value over the region) while one

43 On this see [Verkuyl, 1989] or [Verkuyl, 2005]. The latter paper presumes (familiarity with) a fourfold classification due to Zeno Vendler, in which the performances are subdivided into accomplishments and achievements. An invaluable comparative discussion of Kenny's, Vendler's, Taylor's and other Aristotelian-inspired aspectual taxonomies is provided by [Verkuyl, 1989]. 
of contingency putatively reports heterogeneity. The former cannot be expected to be inherited upwards and the latter cannot expected to be preserved downwards. The same is true for their generalizations (recall the relation between $\mathrm{D}_{0}$ and $\mathrm{D}_{n}$ for $n>0$ from Section 1) as dependence/determination/supervenience and its absence.

All of this can be thought of as a (perhaps laboured) amplification of what Goranko and Kuusisto said in the passage quoted from [Goranko and Kuusisto, 2018] at the start of this section, in particular in these words: "We then argue that, despite both $p \vee q$ and $\mathrm{D}\left(p^{\prime} ; q^{\prime}\right)$ having a reasonable semantics in $\mathcal{D}$, formulae $\mathrm{D}(p ; q) \vee \mathrm{D}\left(p^{\prime} ; q^{\prime}\right)$, which combine $\checkmark$ and $D$, are problematic." The "plus' semantics (and its various incarnations) for $\vee$ seems reasonable in isolation because one thinks initially only of the case in which the disjuncts are homogeneous or 'flat', but the D connective destroys this feature, even if it is imposed for the atomic formulas and inductively inherited by the more familiar modes of composition (though recall that $\neg$ had to be quarantined to pre-atomic position and even then was prone to gappiness or partiality).

In fact something like disjunction itself, as treated by the 'plus' semantics, would raise issues of homogeneity - namely the result of removing all references to 0 in the semantics and disallowing the empty region of logical space. This would ruin $\vee$-introduction (Proposition 4.3(i)) and the downward direction of the Plus Lemma (the 'only if' half of Lemma 4.1) for the same reason as the cases canvassed above, but one can imagine such a proposal made for natural language disjunction by someone objecting to the orthodox pragmatic as opposed to semantic treatment of (what this orthodoxy calls) the implicature of ignorance as to which disjunct is true by the maker of a disjunctive assertion. That is, an epistemic possibility would be held to verify (or justify the assertion of) $\varphi \vee \psi$ just in case it could be decomposed into (possibly overlapping) genuine non-empty possibilities respectively verifying $\varphi$ and $\psi$. Somewhat similar moves have indeed been made in the literature on epistemic and deontic modals, in which the disjunctions are suitably modalized, though we do not go into this here. ${ }^{44}$

44 Accounts which pay special attention to separating out the disjuncts of modalized disjunctions include [Cariani, 2013; Simons, 2005; Zimmermann, 2000]. Zimmerman's idea, in particular, is that $\varphi$ or $\psi$ is rather well represented by something of the form $\diamond \varphi \wedge \diamond \psi$ - compare the discussion of 'Dyirbal disjunction' in pp. 795-798 of [Humberstone, 2011], as well as the discussion of Zimmerman in p. 793f. therewhich would cost us both $\vee$-introduction and $\vee$-elimination for or. The $\vee$-elimination 
The introduction of the 'plus' semantics in [Humberstone, 1988a] was accompanied by the remark (p.65) that it "may look suspiciously conjunctive for a treatment of disjunction," with an explanation that the presence of 0 in the models, verifying everything, should soften any adverse reaction on these lines, and we conclude the present discussion with a 0 -eschewing variation like that in the preceding paragraph though aimed not at disjunction but at temporal conjunction. Here one thinks of the elements of the models as (non-empty) temporal intervals (whether composed of points or not ${ }^{45}$ ) and of $\varphi \wedge^{\bullet} \psi$ as true over (or w.r.t.) an interval $x$ just in case that interval is the concatenation of intervals $y$ and $z$ with $\varphi$ true over $y$ and $\psi$ over $z$, so that the compound $\varphi \wedge^{*} \psi$ gives a reasonable rendering of " $\varphi$ and then $\psi$." 46 Here concatenation is (unlike $\cup$ or + as originally conceived) a partial operation, defined only for pairs of adjacent or abutting intervals. ${ }^{47}$ Adapting the above example of alternating bouts of sunbathing and swimming, so that there

failure is not such a worry because the or-constructions of interest are not assumed to be 'exhaustive' (or 'closed'). As to the fact that or, so treated, is not idempotent, those favourably disposed towards Zimmermann-like treatments may plead that $\varphi \operatorname{or} \varphi$ should not even be regarded as well-formed, or is at worst a 'don't care' case, because of a violation of Hurford's constraint disallowing disjunctions with disjunct-todisjunct entailments - see [Roelofsen, 2016; Singh, 2008]. There is a large literature taking up Zimmermann's (and related) ideas, from which I will mention [AlonsoOvalle, 2005] offering criticism, and [Geurts, 2005] defending a minor modification of the proposal. For many additional references and discussion connecting these issues to the 'inquisitive semantics' of the following two sections, see [Roelofsen, 2016]; see also [Ciardelli, 2016a, p. 249].

45 More generally and more directly parallelling the modal case one would consider arbitrary sets of instants, or for the instant-eschewing, arbitrary fusions of intervals, but intervals are a rather natural choice in the temporal case because of their connection with the temporal ordering, the absence of an analogue of which in the modal case may explain the absence of a modal version of aspect. This candidate explanation must remain provisional and tentative since in several respects the ordering, relative to a fixed world, of worlds by similarity may be thought of as the analogue of the temporal ordering, as in $\$ 5.2$ of [Lewis, 1973]; intervals then correspond to the 'convex propositions' of [Goldstick and O'Neil, 1988] [cf. also Oddie, 1987, commenting on their apparatus]. This modal analogue may underlie the non-temporal uses of still - an idea suggested by (though not quite explicit in) [Michaelis, 1993], thereby unifying them with the temporal uses.

46 For more on this suggestion, which appeared in [Dowty, 1977] and in [Humberstone, 1979] (or originally, [Humberstone, 1974]), see subsection 5.12 of [Humberstone, 2011].

47 And, to repeat, we do not admit the 'empty interval'. Thus a modal analogue of this suggestion might allude to the decomposition of the currently epistemically 
is just one alternation, a correct description of the afternoon might be that you sunbathed $(\varphi)$ and then you swam $(\psi)$, devoting, say, half of the afternoon to each. It does not follow and in the envisaged case that $\varphi \wedge^{*} \psi$ would similarly be a correct description of the first half of the afternoon. Nor, even this was also a correct description of the morning as well, would this be a correct description of the whole day (the 'daytime' day that is). So our $\wedge^{*}$-compounds would not satisfy either the upward or the downward directions of the Plus Lemma (construed with + as concatenation), even if the components satisfied both (as they would be in any stative or homogeneously 'energeia' case). In the terminology of the following section, they would be neither regular nor persistent.

\section{Inquisitive Disjunction}

It would be difficult to improve on the presentation of inquisitive semantics provided in [Ciardelli, 2016b] or on the connection with dependence logic as described there and in [Ciardelli, 2016a], to which the reader is referred for anything beyond the bare minimum summarised here for the sake of looking at the issues raised above. The semantics offered for what are described as classical formulas, constructed [according to Ciardelli, 2016b, p. 143] from sentence letters by means of the primitive connectives $\wedge, \vee, \rightarrow$ and $\perp$, with $\neg \varphi$ taken to abbreviate $\varphi \rightarrow \perp$ is interpreted relative to sets of worlds in much the same way as the second $\Vdash$-truth-definition for the language of $\mathcal{D}$ - the one given with solid rather than hollow bullet points - in Section 3; we make the same change as there from regarding elements of $W$ as truth-value assignments to having this done by a valuation function $V$ in the models $\langle W, V\rangle$, but following [Ciardelli, 2016b], use the $\models$ turnstile and use lower case $s, t$, (for information states) rather than $X, Y$, to range over subsets of $W$. Where $\mathcal{M}$ is such a model, we have the following inductive definition of what it is for state $s$ to support $\varphi$ relative to model $\mathcal{M}$ (" $\mathcal{M}, s \models \varphi$ "); the $\otimes$ notation from [Ciardelli, 2016a] so that we can continue with the "V" of the latter work reserved for the De Morgan defined disjunction:

- $\mathcal{M}, s \models p_{i}$ iff $s \subseteq V\left(p_{i}\right)$

- $\mathcal{M}, s \models \perp$ iff $s=\varnothing$

accessible region of logical space into two non-empty subregions, one verifying $\varphi$ and the other $\psi$ - giving essentially the proposal for $\varphi$ or $\psi$ mentioned in note 44 . 
- $\mathcal{M}, s \models \varphi \wedge \psi$ iff $\mathcal{M}, s \models \varphi$ and $\mathcal{M}, s \models \psi$

- $\mathcal{M}, s \models \varphi \otimes \psi$ iff for some $t, t^{\prime} \subseteq W$ such that $t \cup t^{\prime}=s$ we have $\mathcal{M}, t \models \varphi$ and $\mathcal{M}, t^{\prime} \models \psi$

- $\mathcal{M}, s \models \varphi \rightarrow \psi$ iff for all $t \subseteq s$, if $\mathcal{M}, t \models \varphi$ then $\mathcal{M}, t \models \psi$

The upshot for negation is that in any model a state $s$ supports $\neg \varphi$ just in case every non-empty substate of $t$ fails to support $\varphi$. As Ciardelli remarks (Proposition 1 of [Ciardelli, 2016b]), for all formulas $\varphi$ in this classical fragment, and all models $\mathcal{M}$ and states $s$ thereof, we have the following three properties, the first two of which will be retained while the third is jettisoned (outside the classical fragment) in inquisitive semantics: ${ }^{48}$

(i) Persistence property: if $\mathcal{M}, s \models \varphi$ and $t \subseteq s$, then $\mathcal{M}, t \models \varphi$.

(ii) Empty state property: $\varnothing \models \varphi$.

(iii) Regularity: if $\mathcal{M}, s \models \varphi$ for every $s \in S$, then $\mathcal{M}, \bigcup S \models \varphi$.

Notice that (ii) here is a version of the Zero Lemma recalled in Section 4 (Lemma 4.2) while (i) and (iii) are versions of respectively the "only if" (or "downward") and "if" (or "upward") directions of the Plus Lemma there (Lemma 4.1). They were needed in [Humberstone, 1988a] in order to play a part in the semantics for a non-classical logic (the positive fragment of the relevant logic $\mathbf{R}$ ). The similar behaviour of regions in the possibility semantics touched from for example [Holliday, 2016; Humberstone, 1981] aimed originally not to service the semantics of a non-classical logic but to underwrite a semantics for classically based modal logic without the apparent postulation of fully determinate possible worlds. ${ }^{49}$ The formulas supported by every state in every model with the above semantics in force coincide with the classical tautologies - and indeed the associated consequence relation, defined à la (D3.1) coincides with classical consequence - for the formulas concerned, so it is not for

48 Note that if we considered a Boolean style negation of the kind mentioned in note 30, we would be sacrificing the first-'Persistence'-property. This has been toyed with in essentially the present setting, under the name 'weak negation': Punčochář [Punčochář, 2015].

49 The non-modal fragment had to coincide with classical non-modal logic, of course, and to that end as well as persistence, a condition called refinability was also imposed, but we get this for free when the possibilities/states are taken as sets of worlds. (The reference to Lemma 8.4 of [Holliday, 2016] from Section 4 should be consulted for the general situation.) Failures of regularity did not arise because the treatment of no connective introduced them. 
the sake of a departure from classical logic that this support semantics is being offered; and evidently since the states are themselves taken as sets of worlds, nor is there a motivation to avoid the fully determinate possible worlds. Rather, the reason is given by the rider "for the formulas concerned". We have so far met only the classical formulas, and to fill out the story, we need to cope with the inquisitive disjunctions $\varphi \mathbb{V} \psi$ (or $\varphi \vee_{\mathbf{B}} \psi$ in the notation proposed by Väänänen to extend basic dependence logic) mentioned at the end of Section 4, so we extend the definition of $\models$ with the innocent looking:

- $\mathcal{M}, s \models \varphi \mathbb{V} \psi$ iff $\mathcal{M}, s \models \varphi$ or $\mathcal{M}, s \models \psi$.

And the result is that we lose property (iii) above - regularity - since we can move upwards from states $s, t$ supporting $\varphi, \psi$ respectively, and hence both supporting $\varphi \mathbb{V} \psi$, to $s \cup t$ supporting neither - and therefore not supporting $\varphi \mathbb{} \psi$ - because of what in Section 5 we called the non-homogeneity of this larger set. The loss of regularity is also a loss of flatness, or truth-conditionality as it is called in the present setting (mentioned in note 39 above) and we can see why the latter label might be appropriate when we notice that among the sets of states supporting a given formula there need no longer be a maximal ( $\subseteq$-maximal, that is) such set, since we can no longer just take the union of all supporting states and count on it to support the formula in question. Such maximal states Ciardelli calls alternatives for the formula, and now a formula like $p \llbracket q$ will typically have two alternatives $-V(p)$ and $V(q)$ - and $p \Downarrow q \llbracket r$ (exploiting associativity to suppress parentheses), three. A formula with several alternatives can no longer be thought of as representing a statement to the effect that one of those alternatives obtains since any such statement would subject to regularity: rather it is to be thought as representing a question as to which of those alternatives obtains. Hence the name 'inquisitive disjunction' and also the following suggestive notation: $? \varphi$ for $\varphi \mathbb{V} \neg \varphi$. The semantic value of a sentence in an interpreted language treated along these lines will be a set of downward closed states (for persistence), often in the associated literature called a proposition or an inquisitive proposition - though perhaps this terminology is confusing because the set-of-worlds connotations of "proposition" are by now rather strong - though a variant sometimes employed excludes all but sets of alternatives as just explained. (Some pros and cons, depending on the application, are discussed in [Ciardelli et al., 2014].) The term "state" is replaced by "possibility" in much of this literature. 
As Ciardelli emphasizes in [Ciardelli, 2016b], we can use the interaction of $\rightarrow$ with such questions in order to represent dependencies, and indeed to do so for certain combinations of dependencies which defy expression in the languages of the basic Väänänen-inspired $\mathcal{D}$ and the alternatives reviewed in Section 1 from [Goranko and Kuusisto, 2018] and [Fan, 2016]. First the basic case: $? p \rightarrow q ?{ }^{50}$ If we look at what it takes for a state $s$ supports this formula (in a model) we see that this is that every substate of $s$ supporting ? $p$ should support ?q: that is, for any such substate $t$ for which we have $t \models p$ or $t \models \neg p$, we must have $t \models q$ or else $t \models \neg q$. It is not hard to see that this is equivalent to saying that any worlds (in $s$ ) which agree in respect of membership in $V(p)$ must agree in respect of membership in $V(q)$, which is what $\mathrm{D}(p ; q)$ says (interpreted with $s$ as the set of worlds at issue). But there are now new expressive opportunities.

In the discussion leading up to Remarks 1.7 we saw that, putting the matter in terms of property supervenience, there is not in general for arbitrary properties $P, Q$ a property $P \circ Q$, say, such that for all properties $R, R$ is supervenient on $\{P \circ Q\}$ iff $R$ is supervenient on $\{P, Q\}$. Here we use the $\circ$ notation from the corresponding discussion in Section 2 , where we noted that even weakening the "iff" involved here to "only if" still yields a condition on $P \circ Q$ that is not satisfiable. In particular, $\cap$ would not satisfy this condition on $\circ$ : whenever individuals are alike in respect of $P \cap Q$ they are alike in respect of $P$ and alike in respect of $Q$. Returning to the sentential setting of Section 1, note 10 and the text to which it was appended, there is not only no formula $\chi$ for any given $\varphi_{1}, \varphi_{2}$ such that (for arbitrary models $\left.\mathcal{M}\right) \equiv_{\chi}^{\mathcal{M}}=\equiv_{\varphi_{1}}^{\mathcal{M}} \cap \equiv_{\varphi_{2}}^{\mathcal{M}}$, there is

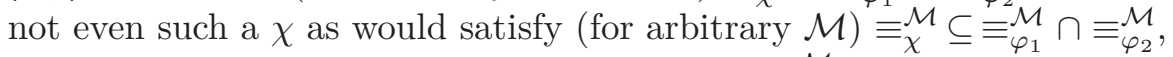
basically because all the equivalence relations $\equiv_{\chi}^{\mathcal{M}}$ are bipartite. Thus we had to have $\mathrm{D}$ be a multigrade connective or else recognise an infinite sequence of separate fixed-arity connectives $\mathrm{D}_{0}, \mathrm{D}_{1}, \ldots, \mathrm{D}_{n}, \ldots$. The situation in the inquisitive setting is quite different, because as well as formulas like $?(p \wedge q) \rightarrow ? r$ - simple question-to-question implications we can form more complex implications and in particular, as Ciardelli amply illustrates in [2016a; 2016b], and elsewhere, distinguish the implication just cited from $(? p \wedge ? q) \rightarrow ? r$ which will now do the work of $\mathrm{D}_{2}(p, q ; r)$, in $\mathcal{D}$ (written by Väänänen as $\left.=(p, q, r)\right)$ - an observation

50 For a similar idea, see the inset implicational formula on p. 307 of [Japaridze, 2006], and the surrounding talk of reducing one (computational) problem to another. 
credited by Ciardelli to Yang [2014] (see now also [Yang, 2017]) — with $\square((? p \wedge ? q) \rightarrow ? r)$ doing the work of $\mathrm{D}_{2}(p, q ; r)$ as understood in Goranko and Kuusisto's $\mathcal{L}_{\mathrm{D}}$, though of course they do not include the subscript "2" (and of course these points hold for arbitrary $\mathrm{D}_{n}$, using instead $n$ inquisitive conjuncts). ${ }^{51}$ The reader can confirm these claims of crosslinguistic equivalence by working through the semantics for $\rightarrow$ described above, and read about its further repercussions (implications with interrogative antecedents and declarative consequents, and exhibiting the converse pattern for instance) in [Ciardelli, 2016b] and other works cited. Since our main concern is with dependence and disjunction, we turn our attention to the latter connective.

Or rather, connectives - since we have (1): the " $\vee$ " of referred to in [Ciardelli, 2016a] as classical disjunction (recall our discussion of this terminological issue at the end of Section 3) and defined De Morgan style from $\neg$ and $\wedge$ (with $\neg \varphi$ itself defined as $\varphi \rightarrow \perp$ ); (2): team disjunction with its 'plus' style semantics (called by Ciardelli tensor disjunction, as remarked in note 36) written in [Ciardelli, 2016a] as $\otimes$; and also (3): $\mathbb{V}$, the specifically inquisitive or question-forming disjunction, alias Väänänen's $\vee_{\mathbf{B}}$. Here is [Ciardelli, 2016a, p.170f.] on the distinction between (1) and (2):

[W] hen applied to truth-conditional formulas $\alpha, \beta$, tensor returns a truth-conditional formula $\alpha \otimes \beta$ which is equivalent to the classical disjunction $\alpha \vee \beta$. This means that, in setting up a support-based implementation of classical logic we could have used $\otimes$ as our classical disjunction rather than defining disjunction in terms of other connectives. This option is indeed pursued in [Ciardelli, 2016b]. While $\otimes$ and $\checkmark$ coincide on statements, however, they yield different results when it comes to questions. The effect of $\vee$ on questions is very dull: since $\varphi \vee \psi$ abbreviates the negation $\neg(\neg \varphi \wedge \neg \psi)$, and since negations are always statements, $\varphi \vee \psi$ is always a statement [...] On the other hand, $\otimes$ does allow us to combine two questions to yield a new question. To see what effect $\otimes$ has on questions, consider first an example: We have:

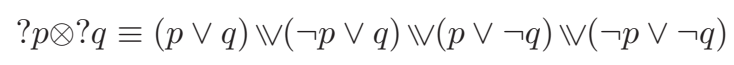

That is, ?p $\otimes ? q$ is a question that is settled as soon as we provide a disjunction of a resolution of ? $p$ with a resolution of ? $q$.

51 See [Ciardelli, 2016a, pp. 164 and 238]. In the former case, while Väänänen sees his "dependence atoms" as statements, Ciardelli's perspective has them on the 'question' side of the divide, making the fact that they are not regular rather more naturally intelligible: see note 8 on p. 168 of [Ciardelli, 2016a]. 
Thus though truth-conditionally equivalent in the sense of having the same support conditions relative to unit sets of worlds, all three disjunction are non-equivalent in general: no two of them have the form compounds with the same support conditions relative to arbitrary states. The specifically truth-conditional formulas (alias 'flat') formulas are those supported by exactly the unions of supporting singletons, and as Ciardelli here remarks, $\neg$ converts any formula into such a formula, with (as he emphasizes elsewhere) $\neg \neg$ converting $\varphi$ into a formula supported exactly by the unions of the singletons supporting $\varphi$. Thus, even if "the effect of $\vee$ on questions is very dull," the fact that it has this effect is not itself quite so dull, since it means that $\vee$ is not itself idempotent: $\varphi \vee \varphi$ amounts to $\neg \neg \varphi$, via its equivalence with $\neg(\neg \varphi \wedge \neg \varphi)$ and the fact that $\wedge$ itself is idempotent. Here by the idempotence of a binary connective $*$ is meant that for any $\varphi$, in any model, a state supports $\varphi$ iff that state supports $\varphi * \varphi$. Thus since "double negation elimination" fails for formulas constructed with the aid of $\mathbb{V}$ - in particular when this is the main connective there will be an barrier to idempotence when $\varphi$ is such a formula. Although Ciardelli [2016a] discusses (a precursor to) Goranko and Kuusisto [2018], he does not specifically address their 'idempotence objection' to Väänänen [2008], so we tease out some of the repercussions of inquisitive semantics for this issue. The failure of $\neg \neg \varphi$ to have $\varphi$ as a consequence in general is something Ciardelli is very explicit about, however, noting that since $\neg \neg p$ has $p$ as a consequence, so that we have a failure of uniform substitution for the set of valid formulas (in this case put an $\rightarrow$ from the former to the latter), or of substitution invariance for the associated consequence relation (given in (D6.1) on p. 59). This principle does hold when any truth-conditional formula is substituted for $p$, and of course one could investigate the logic (closed under uniform substitution) one gets by treating the sentence letters as capable of having the semantics values an arbitrary formula can have, thereby landing, somewhat surprisingly, on an independently well-known (though rather unmanageable) intermediate logic - see [Ciardelli, 2009] and [Ciardelli and Roelofsen, 2011]. (As mentioned in note 38, for this purpose it is $\mathbb{V}$ that should be written as $\vee$, with the present $\vee$ and $\otimes$ discarded. The law of excluded middle $p \vee \neg p$ now becomes the question ?p, certainly not supported/settled by every state - even if by every singleton state in every model.)

To summarize the situation with idempotence it will help to make explicit use of the consequence relation in the background of this discus- 
sion. Accordingly, in the style of (D3.1) on p. 37 - indeed changing only the notation - we have, taking the models, as there, to be of the $\langle W, V\rangle$ form:

(D6.1) $\varphi_{1}, \ldots, \varphi_{n} \models \psi$ iff for every model $\mathcal{M}=\langle W, V\rangle$ and all $s \subseteq W$, if $\mathcal{M}, s \models \varphi_{i}$ for each $i \leq n$, then $\mathcal{M}, s \models \psi$.

Then for our three disjunctions, in the notation currently in play:

- We do not have $\varphi \vee \varphi \models \varphi$ for all $\varphi$.

- We do not have $\varphi \otimes \varphi \models \varphi$ for all $\varphi$

- We do have $\varphi \mathbb{V} \varphi \models \varphi$ for all $\varphi$.

All our disjunctions are $=$-consequences of their disjuncts, so it is the facts listed here on which the fate of idempotence rests, as in Section 4, where it was the second of the above facts that was held by Goranko and Kuusisto to tell against Väänänen's dependence logic. (In that section, $\otimes$ was written as $\vee$ - and recall also from note 38 that in some other publications, Ciardelli's " $\vee$ " stands instead for " $\bigvee "$.

As we have already seen, the contrast between $\varphi \vee \psi$ and $\varphi \otimes \psi$ does not arise for truth-conditional (or 'flat') formulas $\varphi, \psi$, and for these the fact that - to use the latter notation - in general $\varphi \otimes \ldots \otimes$ $\varphi(n$ occurrences of $\otimes)$ can be supported by a state without $\varphi$ being supported by that state is an exact reflection of the failure of Ciardelli's regularity condition, taking $S$ in that condition as $\left\{s_{1}, \ldots, s_{n}\right\}$ with each $s_{i}$ supporting $\varphi$. In other words, the failure of this implication is exactly what would be expected if $\varphi$ represents a question. We return to this point below.

In the quotation from a section of [Goranko and Kuusisto, 2018] entitled "Natural language and logics of determinacy and independence" given at the start of Section 5, we found Goranko and Kuusisto prefiguring their idempotence objection against Väänänen - that any binary connective deserving the name of (inclusive) disjunction form a compound when applied to a formula $\varphi$ taken twice should be fully interreplaceable with $\varphi$ itself salva validitate - in the following words: "We then argue that, despite both $p \vee q$ and $\mathrm{D}(p ; q)$ having a reasonable semantics in $\mathcal{D}$, formulae $\mathrm{D}(p ; q) \vee \mathrm{D}\left(p^{\prime} ; q^{\prime}\right)$, which combine $\vee$ and $\mathrm{D}$, are problematic." The problem was essentially that the $\vee$ their in play $-\otimes$ in the current discussion - did not pass the idempotence test when $\varphi$ was a D-formula, and the simplest case of this problem arises with the disjunction $\mathrm{D} q \vee \mathrm{D} q$, 
which corresponds to $? q \otimes ? q$ in the inquisitive idiom. Goranko and $\mathrm{Ku}-$ usisto based their case for the idempotence condition on a concern with fidelity to natural language - taking it that the word "or" (along with its analogues in other languages) satisfies this condition. (This involves downplaying Hurford's constraint from note 44 above.) But whatever may be said of Väänänen's dependence logic, the motivation behind inquisitive logic was to pay more attention to natural language than logicians had hitherto done, and in particular to correct the imbalance in attention paid to statements at the expense of questions and the almost total absence of attention to logical relations among questions as well as between statements and questions. ${ }^{52}$ The title of [Ciardelli, 2016b], 'Dependency as Question Entailment', uses entailment as a neutralw.r.t. the statement/question contrast - term for the relation holding between $\varphi$ and $\psi$ when any state supporting $\varphi$ supports $\psi$, thereby coinciding with entailment as usually understood - give or take some familiar qualms extraneous to our present concerns - when $\varphi$ and $\psi$ are statements, and to a relation of dependency when both are questions since supporting a question is a matter of supplying an answer (internalized as a connective of the object language in the various logics of dependence, determinacy and supervenience reviewed in our opening sections), and to what is traditionally called presupposition when $\varphi$ is a question and

${ }^{52}$ Here inquisitive semantics should be understood as in our discussion and in all references outside of the present note, and in particular as excluding the development dubbing itself "radical inquisitive semantics" which rather downplays the statement/question distinction and the corresponding distinction between declarativeinternal disjunction and inquisitive disjunction, as well as introducing a negation-like connective - written as " $\div$ " in [Groenendijk and Roelofsen, 2010] which does not have a flattening or truth-conditionalizing effect, making for a more symmetrical treatment of statements and questions. Answering an alternative question is assimilated to agreeing with a disjunctive statement by saying "Yes" and then asserting one of its disjuncts. (Compare [Humberstone, 1998b].) The combined semantic treatment of conjunction, disjunction, and the new negation, bears a close resemblance to the treatment of these ingredients in [Van Fraassen, 1969, p. 484]. (A similarity between the logical upshot of the semantics and Nelson's logic with strong negation/constructible falsity is noted already in [Sano, 2015], one of the few discussions of this topic in print as of the time of this note is being written; another is provided by Aher [2011]. The analogy with Nelson is fair enough since at this stage intuitionistic negation and implication are still in play. Kit Fine has mentioned on several occasions the close relation of inquistive semantics to his own favoured 'exact truthmaker' semanticse.g., in note 1 of [Fine, 2014].) Another approach close to (non-radical) inquisitive semantics is the 'alternative semantics' represented by some of the papers cited in note 44; some comparative remarks on this score can be found in [Roelofsen, 2013]. 
$\psi$ a statement. But there is some rough handling of what might be considered the relevant linguistic data in this admirable synthesis. The fact that there is no natural language construction of negating a question, to correspond to formulas such as $\neg ? p$ is acknowledged by Ciardelli in [2016a], for instance, and one might worry on this score about whether there is any or-construction corresponding to $p \otimes ? q$, or even $? p \otimes ? q$, the latter involving the very occurrence of (a kind of) disjunction about which linguistic intuitions would need to be consulted to defend or to criticize the inquisitive incarnation of Goranko and Kuusisto's complaint. We are familiar with the ambiguity, in the absence of suitable intonational cues, of "Did you see Peter or Quentin?" as between an alternative question $(p \backslash q)$ and a polar - or yes-no - question $(?(p \vee q))$ or with the latter having $\otimes$ instead, equivalently since $p$ and $q$ are truth-conditional. But ? $p \otimes ? q$ attempts to use one these familiarly question-internal "or"s with two questions in its scope. This again is a well-known potential objection (raised by Anna Szabolcsi) to the inquisitive semantics project, discussed in [Ciardelli et al., 2015b], p.80ff. So there is far from being a clear-cut victory to either side on the basis of appeal to the linguistic data-Goranko and Kuusisto on the one hand in whose $\mathcal{L}_{\mathrm{D}}$ only truth-conditional/flat formulas appear, and Väänänen style dependence logic or its distinctively inquisitive development in which the semantics features an irreducibly team/state based support relation. So far we have considered only the appearance in this development of $\mathbb{V}$ to make questions by disjoining the alternatives among which the answerer is to choose, and implications involving such questions. Further issues arise when these questions are embedded under intensional operators and it is to these we devote much of the following section.

Although, as remarked above, Ciardelli does not explicitly address Goranko and Kuusisto's idempotence objection, he does compare their account with his (subsection 6.7.2 of [Ciardelli, 2016a]), and there is a fairly straightforward response (foreshadowed above) to the objection implicit in the discussion. The reference to $\mathrm{G} \& \mathrm{~K}$ is to Goranko and Kuusisto in the following passage [2016a, p. 239] in which Ciardelli compares their approach with that of inquisitive logic:

The central difference between the two systems is that in G \& K's system, dependency is considered as a relation between statements, while in our system it is construed as a relation between questions. The advantage of G \& K's approach is that the semantics of the system can be kept completely truth-conditional. No detour at the level of 
information states is necessary to compute the semantics of dependence statements.

Naturally Ciardelli goes on to explain the disadvantages of the GorankoKuusisto approach, which are precisely repercussions of the failure to put questions centre stage - as was also the case for the comparison with Väänänen's modal dependence logic $\$ 5.2$ of [Ciardelli, 2016a]: we cannot by restricting attention to statements, handling anything other than polar questions, and cannot represent, for instance (Ciardelli's example, pp. 166 and 239):

"What weekday it is determines whether Alice or Bob is in the office." And where mo, tu we th and $f r$ are statements to the effect that it is Monday, Tuesday, etc., we can form the alternative question

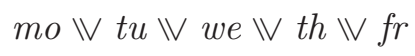

which asks whether it is Monday, Tuesday, or ... or Friday. Similarly we can form the question as to whether Alice or Bob is in the office: $a \Vdash b$. Then we can form the implication:

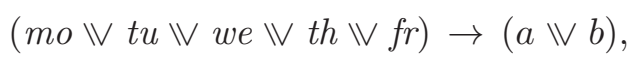

to express the dependency in question. ${ }^{53}$ Evidently the expressive possibilities expand still further with the addition of wh-questions in quantified inquisitive logic, not covered in our discussion.

Returning, then, to the idempotence objection, let us bear in mind that if $\varphi$ and $\psi$ are truth-conditional formulas, their team/tensor disjunction $\varphi \otimes \psi$ is another truth-conditional formula true at exactly the same worlds as at least one of its disjuncts is true at. So the only failures of idempotence arise from the case in which $\varphi$ is not truth-conditional, and in the simplest (but highly representative) case when $\varphi$ has the form

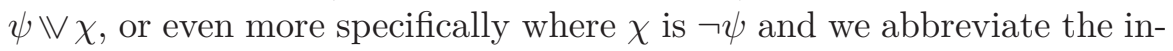
quisitive disjunction to ? $\psi$. Here we do not expect to have ? $\psi \otimes ? \psi \models ? \psi$ simply because a state which is the union of states supporting-i.e., uniformly answering, one way of the other - the question as to whether $\psi$ will not in general itself be a state supporting this question since the uniform answers provided by the substates need not agree. This point was already made above, with $\left\{s_{1}, \ldots, s_{n}\right\}$ rather than just the $n=2$

53 It would be nice to be able to say "report the dependency in question" but see note 51 and the references given there. 
case: to deal with questions, we need to relax the condition of regularity, and the inevitable consequence of that is a failure of idempotence for $\otimes$. But as the brief mention of linguistic intuitions above suggests, it is not as though this theoretically occasioned failure is in tension with any intuitive semantic verdicts since beyond iteration of inquisitive disjunction (for stacking alternatives), it is not clear that we have a way of disjoining questions, so $\otimes$ and $\vee$ may simply fail to put in an appearance at problematic locus of occurrence and there is nothing for linguistic intuition to engage with. ${ }^{54}$

It is worth noting what may seem to be a failure of idempotence, not for $\otimes$ but for $\mathbb{V}$ itself, and coming in the reverse direction: querying whether we should be happy that $\varphi \models \varphi \llbracket \varphi$. Such a query might arise from the facetious (and I would guess fairly recent) appearance of what we might call pseudo-alternative questions such as "Am I good, or am I good?" Since an alternative question is only supported by a state that supports one of its alternatives, any state supporting this question, construed as $\varphi \mathbb{V} \varphi$ will be one which supports $\varphi$, so the question presupposes, or as Ciardelli would put it, entails that the speaker is indeed good in whatever the contextually relevant respect might be-perhaps he or (s)he has just executed a difficult athletic manoeuvre - which is why the question is heard as a boast. But the ordinary question; "Am I good?" has no such entailment. Therefore, the query continues, how can "Am I good?" should not be held to have as a consequence "Am I good or am I good?" and idempotence for $\mathbb{V}$ " fails.

The reply is obvious enough: we have no case here of a failure of $\varphi \models \varphi \mathbb{V} \varphi$, since with $p$ for the statement that the speaker is good "Am I good?" is $p \llbracket \neg p$ while the second is $p \llbracket p$, and the fact that $p \Downarrow \neg p \not \models p \Downarrow p$ is no kind of failure of idempotence. (The issue didn't

54 Dummett [1981, p. 337] using "?" as a question-forming device, writes: "If I am asked, '?(A or B)', then I am required to say 'Yes' if it is the case that either $\mathrm{A}$ or B, and 'No' if it is the case that neither A nor B. But if I am asked '(?A) or (?B)', then I am required either to answer the question '?A' or to answer the question '?B' (or both, if the 'or' is inclusive)." Far from corresponding to the contrast

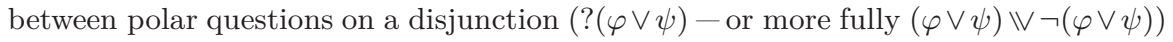
and (other) alternative questions $(\varphi \vee \psi)$, Dummett has in mind in the latter case examination questions allowing a choice as to which part to answer, which he has just illustrated with a case in which both parts were, incidentally, wh-questions, and the type-setting itself - each question starting with a capital letter and with a small caps "OR" between them on a separate line-makes abundantly clear what a marginal and metalinguistic construction is involved here. 
really concern idempotence specifically, anyway, since "Am I good?" also fails to have as a consequence "Am I good or is Spitzbergen inhabited?", which might have been held to threaten or-introduction for $\mathbb{V}$.)

As a last point about inquisitive disjunction before we turn to its embedding in epistemic contexts in the following section: From the discussion after Remark 4.4, we know that since $\mathbb{V}$ forms disjunctive combinations on the right of the relational connection engendered by any model (with $\models$ as the binary relation between states of the model and formulas), there cannot in general be conjunctive combinations on the left (since we the cross-over condition is not generally satisfied). That is, there cannot be a binary operation ', say, on states such that for any model $\mathcal{M}$ with universe $W$, we have for all formulas $\varphi$ :

For all $s, t \in W, \mathcal{M}, s \cdot t \models \varphi$ iff $\mathcal{M}, s \models \varphi$ and $\mathcal{M}, t \models \varphi$.

In other words, it's not just that we can't take - as union for states: there is no alternative candidate either. And once we do take - as union, having the 'only if' direction here - persistence - means that we cannot also have the 'if' direction - regularity.

\section{The Wonders of Whether-Disjunction}

Suppose we have a logic in whose language there are (primitive or derived) connectives $\#_{1}, \#_{2}$, and $\#_{3}$ of arities respectively $1, n$ and $n$, for some $n$. (We use 'infix' notation for the latter pair). In the terminology of [Humberstone, 2011, p. 559f.], \# 3 is said to be an intraposed version of $\#_{2}$ (across $\#_{1}$ ), and $\#_{2}$ an extraposed version of $\#_{3}$ according the logic in question if for all formulas $\varphi_{1}, \ldots \varphi_{n}$ of its language, the formulas

$$
\#_{2}\left(\#_{1} \varphi_{1}, \ldots, \#_{1} \varphi_{n}\right) \text { and } \#_{1} \#_{3}\left(\varphi_{1}, \ldots, \varphi_{n}\right)
$$

are freely interreplaceable in the logic. For example, with $n=2$, conjunction is an extraposed version of disjunction across $\#_{1}=\neg$ according to intuitionistic logic, and vice versa as well according to classical logic (De Morgan). When discussing this in connection with monomodal logic, however, the default assumption will be that (unless otherwise stated) $\#_{1}$ is the operator $\square$. Thus an intraposed version of $\left(\#_{2}=\right) \vee$ according to a normal (indeed any congruential) such logic would be a binary connective $\#_{3}$ - he written in 'infix' position - for which the logic contains all formulas of the form

$$
(\square \varphi \vee \square \psi) \leftrightarrow \square\left(\varphi \#_{3} \psi\right) .
$$


For a reason recalled below, an intraposed version of disjunction like this is referred to as representing whether-disjunction in [Humberstone, 2011]. Note that if a normal modal logic provides an intraposed version of $\vee$ across $\square$, then it also provides an intraposed version - here written as $*$ - of $\wedge$ across $\diamond$, since $(\diamond \varphi \wedge \diamond \psi) \leftrightarrow \diamond(\varphi * \psi)$ will be provable when $\varphi * \psi$ is taken as $\neg\left(\neg \varphi \#_{3} \neg \psi\right)$. While some of the examples treated below are easier to understand when formulated in the latter terms (such as Example 7.1, for instance), we retain the $\square$-orientation (with "across $\square$ " tacit) in what follows to stay close to the passages quoted from [Ciardelli, 2016a].

As Example 4.33 .13 of [Humberstone, 2011] - repeated again as Example 7.1 here-illustrates, introducing an intraposed version of disjunction in the setting of normal modal logic poses a risk of nonconservative extension. That example shows, in particular, that the logic $\mathrm{K} 4_{c}$ (i.e., the smallest normal modal logic containing all formulas of the form $\square \square \varphi \rightarrow \square \varphi$ : see note 85) is extended nonconservatively by the addition of a binary connective \# (we can safely drop the subscript " 3 " now) for which $\square(\varphi \# \psi)$ is equivalent to $\square \varphi \vee \square \psi$ for all formulas $\varphi, \psi$; this equivalence would allow one to read " $\varphi \# \psi$ " as "whether $\varphi$ or $\psi$ " if $\square$ is read as "so-and-so knows (that)". .55

EXAMPLE 7.1. The idea is that while $\square(\square \varphi \vee \square \psi) \rightarrow(\square \varphi \vee \square \psi)$ is not $\mathrm{K} 4_{c}$-provable for arbitrary $\varphi, \psi$, the result of adding \# as an intraposed version of $\vee$ makes available proof of all instances - including those in which \# does not itself appear - of this schema. (In more detail: we use a special case of the $4_{c}$ schema, $\square \square(\varphi \# \psi) \rightarrow \square(\varphi \# \psi)$ and then reformulate the antecedent and consequent in accordance with the equivalence stipulated to hold for necessitated \#-compounds.) The same example would work to show that $\mathrm{K} 4$ ! ( $=$ the smallest normal modal logic containing all formulas of the form $\square \varphi \leftrightarrow \square \square \varphi$ ) is likewise extended nonconservatively by the addition of a whether-disjunction forming operator \#. Similar reasoning shows that the analogous extension of the smallest normal modal logic containing all instances of the schema $\square \square \varphi \rightarrow \square \square \square \varphi$ would likewise yield the previously underivable schema $\square(\square \varphi \vee \square \psi) \rightarrow \square \square(\square \varphi \vee \square \psi)$.

55 Of course when followed by whether, the that after knows would be dropped. Some less abstract issues in connection with knowing whether are raised under Example 5.1.2 of [Humberstone, 2016, (p. 334f.], as well as other places there as directed by the index entry for that phrase. 
In view of the potential nonconservativity of the whether-disjunction extension of a normal modal the apparent use of $\mathbb{V}$ as just such a disjunction in [Ciardelli, 2016a] naturally raises the question as to how this is manages to be viable option at all. And although Väänänen does not draw attention to it, the materials of his [2008] also provide for such a connective, though with a subtle difference. In the remainder of this section we address this question as first as it applies to Ciardelli and Väänänen, beginning for expository reasons with the former, and then turn our attention, since the subject has a certain general interest, to a few further observations concerning normal modal logics on the standard (rather than inquisitive or dependence logical) approach as to the conservativity of extending them with whether-disjunction.

First then, let us look at Ciardelli's discussion. The passage quoted below is from Example 6.2.4 of [Ciardelli, 2016a, p. 204]. The treatment of $\square$ as the main connective of a formula being evaluated relative to a state (set of worlds) $s$ we keep under wraps for the moment, since it is not needed for following the passage quoted here (or the one after that), while its treatment as evaluated relative to a world $w$-i.e., the special case in which $s=\{w\}$, is given by:

$$
\mathcal{M}, w \models \square \varphi \Longleftrightarrow \mathcal{M}, R(w) \models \varphi,
$$

as in [Goranko and Kuusisto, 2018] (see Section 5 above). In this passage Ciardelli writes " $\left|p_{i}\right|_{\mathcal{M}}$ " for $V(q)$ where $V$ is the valuation component of the model $\mathcal{M},{ }^{56}$ and $\sigma(w)$ for $R(w)$ :

$[\ldots]$ consider the formula $\square(p \Downarrow q)$. We have:

$$
\begin{aligned}
\mathcal{M}, w \models \square(p \Downarrow q) & \Longleftrightarrow \mathcal{M}, \sigma(w) \models p \Downarrow q \\
& \Longleftrightarrow \sigma(w) \subseteq|p|_{\mathcal{M}} \text { or } \sigma(w) \subseteq|q|_{\mathcal{M}}
\end{aligned}
$$

Thus, $\square(p \Downarrow q)$ is true at $w$ iff the state $\sigma(w)$ settles a specific one among $p$ and $q$. Under an epistemic interpretation, we may take this to capture the fact that the agent knows whether $p$ or $q$.

On the following page of [Ciardelli, 2016a] this is generalized to arbitrary formulas of the language in Proposition 6.2.7 whose content is that for any formulas $\varphi, \psi$, the formulas $\square(\varphi \vee \psi)$ and $\square \varphi \vee \square \psi$ are supported by

${ }^{56}$ Or more generally, since $\varphi$ need not be atomic, $|\varphi|_{\mathcal{M}}$ for the set of points in $\mathcal{M}$ at which $\varphi$ is true'; Ciardelli in fact writes $M$ rather than $\mathcal{M}$ for the models, so the quotation to follow is not quite verbatim. 
exactly the same states (in any given model); the proof runs as follows (p. 206):

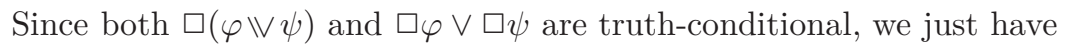
to show that they have the same truth-conditions. We have:

$$
\begin{aligned}
\mathcal{M}, w \models \square(\varphi \mathbb{V} \psi) & \Longleftrightarrow \mathcal{M}, \sigma(w) \models \varphi \mathbb{\vee} \psi \\
& \Longleftrightarrow \mathcal{M}, \sigma(w) \models \varphi \text { or } \mathcal{M}, \sigma(w) \models \psi \\
& \Longleftrightarrow \mathcal{M}, w \models \square \varphi \text { or } \mathcal{M}, w \models \square \psi \\
& \Longleftrightarrow \mathcal{M}, w \models \square \varphi \vee \square \psi .
\end{aligned}
$$

And finally, between the two passages just quoted, Ciardelli provides the following interesting discussion. Note that in the opening sentence "universal modality" does not mean (as in Goranko and Kuusisto [Goranko and Kuusisto, 2018] and other publications by Goranko) "operator whose accessibility relation in any model is the universal relation on that model" but rather "operator whose semantic interpretation is understood in terms of universal (as opposed to existential) quantification over accessible points":

We have thus illustrated what sort of facts one can express by letting the universal modality $\square$ apply to questions. In standard modal logic it is not possible to apply a modal operator to a question - since the language contains no formulas expressing questions. The fact that an agent knows whether $p$ can, nevertheless, be expressed by means of the paraphrase $\square p \vee \square \neg p$. In our system, in which questions can be embedded within modalities the equivalence $\square ? p \equiv \square p \vee \square \neg p$ is obtained as a logical fact, rather than stipulated by definition.

It is hard not to share some enthusiasm at having the significance of " $\square$ ?p" (" $\square(p \Downarrow \neg p)$ ") - and in particular its equivalence with " $\square p \vee$ $\square \neg p$ " - fall out from a general account of $\square$ and $\mathbb{\vee}$, but unwanted side effects may dampen this enthusiasm. For example modal principles holding for all truth-conditional formulas may have substitution instances as explained in the preceding section - which no longer hold with the inquisitive $\mathbb{V}$-compounds replacing the declarative sentence letters, making us think twice about whether $\square$ really does have the same significance with the latter in its scope as it does when only the former are (and their truth-conditional substitution instances) are embedded under it. Aside from the uniform substitution issue, we might need to attend to congruentiality or the replacement property for equivalent formulas. In 
the setting of normal modal logics one often considers the stronger monotonicity property for $\square$, i.e., the rule ( $\square$ Mono) under which such logics are closed:

$$
\frac{\varphi \rightarrow \psi}{\square \varphi \rightarrow \square \psi}
$$

(The congruentiality rule would have $\leftrightarrow$ for at least the premiss occurrence of $\rightarrow$.) Any disruption of such replacement properties would again raise a doubt as to whether any enrichment of the language occasioning such a disruption was really consistent with say that $\square$ meant the same after the envisaged extension as it did before - even if the extension was 'conservative' in the familiar sense, ${ }^{57}$ as we saw with Example 7.1 was definitely not the case when we added whether-disjunction and allowed unfettered appeal to these principles of substitution and replacement.

Accordingly, let us revisit the Example 7.1, so that we can pay close attention to the fate of individual steps of a formal derivation of the unwanted result as we bring to bear the semantic apparatus of inquisitive logic. Accordingly we now write \# as $\mathbb{V}$ and to make the use of Uniform Substitution completely clear we proceed with sentence letters rather than schematic letters, and will treat the whether-disjunction equivalence ('WDE' for short) as a concrete formula rather than a schema:

$$
\square(p \Uparrow q) \leftrightarrow(\square p \vee \square q)
$$

and in addition we abbreviate reasoning in accordance with classical truth-functional logic to "TF"; this will be legitimate since the relevant classical principles are instantiated by truth-conditional formulas:

(1) $\square \square p \rightarrow \square p$

(2) $\quad \square \square(p \Uparrow q) \rightarrow \square(p \Downarrow q)$

(3) $\quad(\square p \vee \square q) \rightarrow \square(p \llbracket q)$

(4) $\quad \square(\square p \vee \square q) \rightarrow \square \square(p \Downarrow q)$

(5) $\quad \square(\square p \vee \square q) \rightarrow \square(p \backslash q)$

(6) $\quad \square(p \Downarrow q) \rightarrow(\square p \vee \square q)$

(7) $\quad \square(\square p \vee \square q) \rightarrow(\square p \vee \square q)$ $4_{c}$ $\mathrm{US} p \llbracket q$ for $p$ in (1) $\leftarrow$ half of (WDE)

(3), ( $\square$ Mono)

(2), (4), TF

$\rightarrow$ half of (WDE)

(5), (6), TF

To see what might be going wrong in this derivation, we bring the semantics to bear. Now we need to be explicit about Ciardelli's general

57 The desideratum here is closely related to that referred to [Humberstone, 2011, p. 1233] as conservation of synonymy. 
clause in the definition of the support relation $(\models)$ because even if we start evaluating a formula at a singleton state, if the formula has (as $4_{c}$ has) a $\square$ within the scope of a $\square$, we need to know not just what it takes for a world $w$ to support a formula of the form $\square \varphi$ but also what it takes for the state $R(w)$ to support such a formula. On this matter we have been remaining silent so as to hear Ciardelli's voice without distractions, but because it is precisely here that his treatment of $\square$ differs from that of Väänänen, and the semantics of Väänänen [2008] also tells us that the left and right hand sides of (WDE) are supported by the same sets of worlds, though we recall that Väänänen used the notation $\vee_{\mathbf{B}}$ rather than $\mathbb{V}$. We give the two inductive clauses in the definition of support in Ciardelli's notation here (except with $R(\cdot)$ rather than $\sigma(\cdot)$, rewriting Väänänen's as given near the start of Section 5 above, putting " $s$ " for " $U$ " and " $=$ " for "Iト", so that from here on we can use " $=$ " appropriately signposted by a subscript "C" or "V" depending as to whether the semantics with Ciardelli's clause $(\mathrm{C} \square)$ is operative or Väänänen's clause $(\mathrm{V} \square) ;{ }^{58}$ in the latter, $R(s)$ is $\bigcup_{w \in s} R(w)$ :

$$
\begin{aligned}
& \mathcal{M}, s \models \square \varphi \Longleftrightarrow \mathcal{M}, R(w) \models \varphi \text { for all } w \in s, \\
& \mathcal{M}, s \models \square \varphi \Longleftrightarrow \mathcal{M}, R(s) \models \varphi .
\end{aligned}
$$

First, notice that these give the same result for the case already spelt out above, with $s=\{x\}$. But in the general case they need not coincide, since the rhs of $(\mathrm{C} \square)$ requires each $w \in s$ to have $R(w)$ support $\varphi$, whereas (V $\square)$ asks for the union of these states $R(w)$ to support $\varphi$, which does not follow from each such state supporting $\varphi$ when $\varphi$ if $\varphi$ is not regular, which precisely what is of interest to us here where $\varphi$ in some cases represents the non-truth-conditional formula $p \llbracket q$. We shall see how this difference plays out in detail in what follows. ${ }^{59}$

58 He sources are respectively [Ciardelli, 2016a, p. 202], where a stray " $\alpha$ has accidentally appeared in place of the " $\varphi$ " on the right, and [Väänänen, 2008, p. 244].

59 Ciardelli [2016a] mentions Väänänen's semantic treatment of $\square$ on p. 235 and it is because of Väänänen's prominence in the present discussion that the label "(V $\square)$ " is chosen, though in fact this treatment can be found in the more general 'possibilities' framework (i.e., not requiring that the possibilities be thought of as sets of worlds) in [Humberstone, 1981]; further discussion and references of that approximate vintage appears on p. 359 of [Humberstone, 1988b]. A recent intensive investigation of the options here is supplied in [Holliday, 2014] and other publications. Curiously, Ciardelli [2016a] picks up the latter discussion on p. $240 f$., though without remarking that this 'functional possibility semantics' in the section heading on that page - is the same as the treatment discussed earlier apropos of Väänänen's modal dependence logic. 
Beginning with Ciardelli, we note that [2016a] already sounds a warning about Uniform Substitution in the present setting, though he puts this in terms of schemata at p. 213, noting that if we think of in the inquisitive version of a given normal modal logic $L$ in its standard incarnation as being the set of formulas supported by every state in any model on a frame for $L$, then sometimes a schema all of whose truth-conditional instances are valid - and the inquisitive version does not different in respect of which of these are valid - can have invalid instances in the full inquisitive language because of the presence of $\mathbb{V}$. Rather than thinking of this in terms of schemata and their instances, we can think of it terms of concrete formulas and their substitution instances. With this modulation, Ciardelli's example is of KT, and we repeat essentially the same example here: ${ }^{60}$

EXAMPLE 7.2. KT is the normal modal logic determined by the class of reflexive frames, and this class comprises exactly the frames for this logic. So its inquisitive version will be the set of formulas in the language of the present discussion which are valid on all such frames. Although in the standard treatment all substitution instances of the formula sometimes called $\mathrm{T}, \square p \rightarrow p$ are valid on such frames, if we substitute $p \llbracket q$ for $p$, we get a formula of the present language lacking this property. Top see this, take a model $\mathcal{M}=\langle W, R, V\rangle$ with $W=\left\{w_{1}, w_{w}\right\}, R=\left\{\left\langle w_{1}, w_{1}\right\rangle,\left\langle w_{2}, w_{2}\right\rangle\right.$ - so the frame is certainly reflexive - and $V(p)=\left\{w_{1}\right\}, V(q)=\left\{w_{2}\right\}$; then we will have, choosing $W$ itself as the state in question:

(i) $\mathcal{M}, W \models_{C} \square(p \Downarrow q)$

but

(ii) $\mathcal{M}, W \nvdash_{C} p \Downarrow q$.

We have (i) because for each $w \in W, R(w)$ either supports $p$ (though not $q$ ) or supports $q$ (though not $p$ ), given the choice of $V$, but we have (ii) because $W$ supports neither of $p, q$, since $w_{2}$ (i.e., $\left\{w_{2}\right\}$ ) does not support $p$ and $w_{1}$ does not support $q$.

Remark 7.3. If we work this example using (V $\square$ ) instead of $(\mathrm{C} \square)$, incidentally, we find that we lose (i). To have $\mathcal{M}, W \models_{V} \square(p \Downarrow q)$, we should need $\mathcal{M}, R(W) \models_{V} p \Downarrow \neg p$. But $R(W)=W$ in this case and we have just seen, in (ii), that we do not have this. (There is no difference between $\models_{V}$ and $\models_{C}$ on $\square$-free formulas.)

${ }^{60}$ Ciardelli's own example involves the formula $p \Downarrow \neg p$, alias ? $p$, rather than $p \llbracket q$, but the latter is more convenient for present purposes. 
As Ciardelli emphasizes in [2016a] Uniform Substitution of truthconditional formulas for sentence letters preserves validity. But while the antecedents and consequents of all the implicational formulas in the our derivation (1)-(7) above are truth-conditional formulas (and so therefore are the implications themselves) the actual substitution made in the transition from line (1) to line (2) is of the non-truth-conditional formula $p \Downarrow q$ for $p$. Thus we do not have a blanket guarantee that we have not passed from a formula valid on all reflexive frames to one lacking this feature. And in fact a minor modification of Example 7.2 shows that we done exactly this, which is not surprising since $4_{c}$ is a substitution instance case of $\mathrm{T}$ - though it is perhaps surprising if one compares the case of $\mathrm{T}$ with that of 4 (i.e., $\square p \rightarrow \square \square p$ ) concerning which Ciardelli shows that all of its substitution instances in the inquisitive language are valid on the frames validating 4 (the transitive frames, that is), which prompt the conjecture that it is sufficient, for validity to be inherited by all substitution instances, that we are dealing with a formula which is (like 4 but unlike T) fully modalized. Not so, however:

Example 7.4. We modify the frame $\langle W, R\rangle$ to $\left\langle W^{+}, R^{+}\right\rangle$of Example 7.2 , by taking an something $w_{0}$ distinct from $w_{1}$ and $w_{2}$ and putting $W^{+}=W \cup\left\{w_{0}\right\}, R^{+}=R \cup\left\{\left\langle w_{0}, w_{1}\right\rangle,\left\langle w_{0}, w_{1}\right\rangle\right\}$. Note that this is a dense frame (i.e., one in which $R(x) \subseteq R(R(x))$ for all frame elements $x$, where $R$ is the frame's accessibility relation), and hence a frame for $\mathrm{K} 4$. But consider the model $\mathcal{M}^{+}=\left\langle W^{+}, R^{+}, V\right\rangle$ ( $V$ exactly as in Example 7.2, that is.) The substitution instance we passed to in line (2) of the derivation (1)-(2) is false at $w_{0}$ in $\mathcal{M}^{+}$, with $(\mathrm{C} \square)$ in force, since we have: (i) $\mathcal{M}^{+}, w_{0} \models_{C} \square \square(p \Downarrow q)$ but (ii) $\mathcal{M}^{+}, w_{0} \nvdash_{C} \square(p \Downarrow q)$.

We have (i) because this means $\mathcal{M}^{+}, R\left(w_{0}\right) \models_{C} \square(p \vee q)$, which means that for all $x \in R\left(w_{0}\right)$, we have $\mathcal{M}^{+}, R(x) \models_{C} p \llbracket q$, i.e., since $R\left(w_{0}\right)=\left\{w_{1}, w_{2}\right\}$, both $\mathcal{M}^{+}, R\left(w_{1}\right) \models_{C} p \Downarrow q$ and $\mathcal{M}^{+}, R\left(w_{2}\right) \models_{C}$ $p \llbracket q$, in the first case because $\mathcal{M}^{+}, R\left(w_{1}\right) \models_{C} p$ and in the second because $\mathcal{M}^{+}, R\left(w_{2}\right) \models_{C} q$ (noting that $R\left(w_{i}\right)=\left\{w_{i}\right\}$ for $i=1,2$ ). We have (ii) because to have had $\mathcal{M}^{+}, w_{0} \models_{C} \square(p \Uparrow q)$, we would need $\mathcal{M}^{+}, R\left(w_{0}\right) \models p \llbracket q$, which is not the case because $R\left(w_{0}\right)=\left\{w_{1}, w_{2}\right\}$ and this state supports neither $p$ (since it contains $w_{2}$ ) nor $q$ (since it contains $\left.w_{1}\right)$.

Remark 7.5. Again, with (V $\square$ ) in force instead of (C口), we lose (i), since having

$$
\mathcal{M}^{+}, w_{0} \models_{C} \square \square(p \Uparrow q)
$$


would mean, by two appeals to (V口), having $\mathcal{M}^{+}, R\left(R\left(w_{0}\right)\right) \models_{C} p \llbracket q$. Since $R\left(R\left(w_{0}\right)\right)=\left\{w_{1}, w_{2}\right\}$, this is not the case, as we just saw at the end of Example 7.4.

The story so far, then: On Ciardelli's construal of $\mathbb{V}$ as whetherdisjunction, interpreting the $\square$ in (WDE) by (C $\square$ ), the derivation (1)-(7) has gone wrong already at line (2), in making a prohibited substitution, whereas on Väänänen's construal of it - not that Väänänen concerned himself with precisely this issue - interpreting the $\square$ in (WDE) by (C $\square$ ) by means of (V $\square)$, everything is in order so far, and the mistake must come later, so we proceed to a diagnosis of what exactly goes wrong with this interpretation of $\square$ in force. It will help to expand on the content of Remark 7.5 to see what the antecedent of line (2) in our derivation amounts to as an $\mathbb{V}$-free formula of the object language, as evaluated at a point $w$ (i.e., singleton state) in an arbitrary model $\mathcal{M}$ with accessibility $R$ model; we already know, since (WDE) tells us this, that the corresponding $\mathbb{V}$-free equivalent of the consequent is, namely. We $\square p \vee \square q$. (We could equally well write $\otimes$ rather than $\vee$, but since the disjuncts are truth-conditional, the results are equivalent and we use the more familiar notation.) This is something the $(C \square)$ and (V $\square$ ) semantics agree on. Turning to the antecedent then, we have:

$$
\begin{aligned}
& \mathcal{M}, w \models_{V} \square \square(p \Downarrow q) \Longleftrightarrow \mathcal{M}, R(w) \models \square(\varphi \vee \psi) \\
& \Longleftrightarrow \mathcal{M}, R(R(w)))) \models_{V} p \llbracket q \\
& \Longleftrightarrow \mathcal{M}, R(w) \models_{V} p \text { or } \mathcal{M}, R(R(w)) \models_{V} q \\
& \Longleftrightarrow \mathcal{M}, R(w) \models_{V} \square p \text { or } \mathcal{M}, R(w) \models_{V} \square q \\
& \Longleftrightarrow \mathcal{M}, w \models_{V} \square \square p \text { or } \mathcal{M}, w \models_{V} \square \square q \\
& \Longleftrightarrow \mathcal{M}, w \models_{V} \square \square p \vee \square \square q
\end{aligned}
$$

Thus written in $\mathbb{V}$-free notation, line (2) of the earlier derivation amounts when interpreted via ( $\mathrm{V} \square)$, to the formula

$$
(\square \square p \vee \square \square q) \rightarrow(\square p \vee \square q),
$$

and thus the transition from (1) to (2) evidently keeps us within the formulas valid on all dense frames. ${ }^{61}$ So (at least here) Uniform Substitution has not let us down. But working through the semantics in

61 The implication from the first disjunct of the antecedent to the first disjunct of the consequent is just $4_{c}$, while the implication from the second disjunct of the antecedent to the second disjunct of the consequent is just a re-lettered version of this with $q$ for $p$. 
the case of $\square \square(p \llbracket q)$ above shows us something else of no less concern: the result of prefixing a $\square$ to the $\mathbb{V}$-free representation of $\square(p \Downarrow \vee q)$ is not equivalent to the $\mathbb{V}$-free representation of prefixing a $\square$ to $\square(p \mathbb{\vee} q)$ itself. The first of these is

$$
\square(\square p \vee \square q),
$$

while, as we have just seen, the second of these is $\square \square p \vee \square \square q$. The second formula has the first as a consequence in $\mathrm{K}$ though not conversely - and it is $\mathrm{K}$ that is relevant rather than $\mathrm{K} 4_{c}$ since the present point does not depend at all on restricting attention to frames for the latter logic.

Now, the existence of translations between different logics in the broad dependence/inquisitive logic family which cannot be presented as compositional translations is abundantly evidenced in [Ciardelli, 2016a; Yang, 2014], but here matters stand somewhat differently because we are actually considering the expressive resources of a single language. In particular we are witnessing a failure of congruentiality, since the following application of the congruentiality rule:

$$
\frac{\square(p \Uparrow \vee) \leftrightarrow(\square q \vee \square q)}{\square \square(p \Downarrow q) \leftrightarrow \square(\square q \vee \square q)}
$$

does not preserve validity (on arbitrary frames). Thus the actual step at which our (1)-(7) derivation above goes wrong is in the application of ( $\square$ Mono) in passing from line (3) to line (4). Readers can decide for themselves whether the need to restricted familiar general principles concerning $\square$ in the inquisitive-logical takes some of the wind from the sails of the claim that whether-disjunction equivalence has been "obtained as a logical fact, rather than stipulated by definition". (Remark 5.1 already raised an eyebrow at a failure of ( $\square$ Mono) to preserve validity on one version of Väänänen's semantics.)

A more thoroughgoing general analysis of the differences between the two semantic treatments, (C $\square$ ) and (V $\square$ ) of $\square$ in the dependence/ inquisitive area, and their bearing on different modal formulas, would be desirable, but here we will leave matters with putting the above observations about the interaction between $\square$ and $\mathbb{V}$ in slightly more general terms, and return to the issue of the conservativity or otherwise of adding whether-disjunction in the standard setting of normal modal logic. With ( $\mathrm{V} \square)$ in force, as we have seen, the $\mathbb{V}$-free equivalent of $\square \square(p \Uparrow q)$ is $\square \square p \vee \square \square q$; it is similarly easy to check that $(\mathrm{C} \square)$ in force, the equivalent is instead $\square(\square p \vee \square q)$. More generally, where $\square^{n}$ indicates 
$n$ occurrences of $\square$, consider the two equivalences for all $n \geq 1$, noting that $C(1)=V(1)=(\mathrm{WDE})$ :

$$
\begin{aligned}
& \square^{n}(p \Downarrow q) \leftrightarrow \square^{n-1}(\square p \vee \square q) \\
& \square^{n}(p \Downarrow q) \leftrightarrow\left(\square^{n} p \vee \square^{n} q\right)
\end{aligned}
$$

The point here is that for all $n$, and any model $\mathcal{M}$ and point $w$ therein, $\mathcal{M}, w \models_{C} C(n)$ and $\mathcal{M}, w \models_{V} V(n)$. (Since the formulas concerned are truth-conditional, we can equally well say: "for all states $s .$. ", instead of "for all points $w ", \ldots)$ The two sequences $\overrightarrow{C(i)}$ and $\overrightarrow{V(i)}$ of equivalences fare differently in respect of what happens if we try apply the congruentiality rule to one of their elements - in other words, if we attach a $\square$ to the formula on each side of the $\leftrightarrow$. In the case of the $C(i)$, this is a completely benign operation, simply converting $C(n)$ into $C(n+1)$. But with the $V(i)$, matters are quite different, since this converts $V(n)$ into

$$
\square^{n+1}(p \vee q) \leftrightarrow \square\left(\square^{n} p \vee \square^{n} q\right),
$$

and this is bad news because we already have $V(n+1)$ telling us that the left-hand side of this biconditional is equivalent to a quite different formula, namely $\square^{n+1} p \vee \square^{n+1} q$. Thus replacement of equivalents is prone to cause trouble for the (V $\square$ ) interpretation. It would be pleasant to be able to report a corresponding difficulty is raised by Uniform Substitution in connection with the sequence of the $C(i)$ for the case of the $(\mathrm{C} \square)$ interpretation, but the substitution difficulties encountered for this case do not immediately suggest any such parallel.

For the remainder of this section, we turn back to the familiar environment of normal modal logic - the setting of Example 7.1 - to sample some of the range of normal modal (and bimodal) logics which can be relatively easily seen to be conservatively extended or relatively easily seen to be nonconservatively extended, by the addition of whetherdisjunction. The issue has a rather general issue because in what the conservativity of an extension in this case means is that disjunctions of $\square$-formulas behave exactly as though they were $\square$-formulas in their own right. The positive and negative examples given here may help provide data suggestive for a general account - an informative necessary and sufficient for the conservativity of the extension in question, though we do not attempt such an account here, and will begin with a rather specific condition on frames.

By a generalized equivalence relation on a set $W$ will be meant (as in [Humberstone, 2016, pp. 443-445]) a binary relation $R$ such that, 
writing $R(x)$ for $\{y \in W \mid R x y\}$, for any $w, x \in W$ either $R(w)=R(x)$ or $R(w) \cap R(x)=\varnothing$. When $R$ is a generalized equivalence relation on $W \neq \varnothing$ in this sense we say that the pair $\langle W, R\rangle$ is a frame satisfying the generalized equivalence condition. ${ }^{62}$

Each of the following schemata tense-logically defines (i.e., is valid on precisely) the class of frames satisfying the generalized equivalence condition, and the smallest tense logic ${ }^{63}$ containing all instances of any one of them is sound and complete w.r.t. the class of these frames. Here we have used Prior's $G / H$ notation for $\square$ and $\square^{-1}$ of the preceding footnote, with $F$ and $P$ for the dual operators. This notation saves us from having a lot of " -1 " superscripts:

$$
\begin{array}{rrrr}
G \varphi \rightarrow G H G \varphi & F H \varphi \rightarrow G H \varphi & H \varphi \rightarrow H G H \varphi & P G \varphi \rightarrow H G \varphi \\
F P F \varphi \rightarrow F \varphi & F P \varphi \rightarrow G P \varphi & P F P \varphi \rightarrow P \varphi & P F \varphi \rightarrow H F \varphi
\end{array}
$$

These schemata are arranged with the dual or contraposed form of a given schema vertically aligned with that schema axiomatize the same tense logic. The first and third pairs are (past/future) mirror images, as are the second and fourth. And each such pair locally defines a different property of points within frames, as with the properties of 1-, 2-, and 3transitivity in [Humberstone, 2016, p. 185f.] whose universal possession by all points a frame amounts to the frames satisfying the generalized equivalence condition. There are four local properties here because of the four variables in the explicit formulation of generalized equivalence as the universal closure of

$$
(R w y \wedge R x z \wedge R w z) \rightarrow R x z
$$

For example the either formula from the first pair locally define the property of being an $x$ such that for all $w, y, z$, the above condition is

62 For a discussion of this condition and the closely related cross-over condition from the passage following Remark 4.4 above, see [Humberstone, 1991]. ([Humberstone, 1995b] includes follow-up, errata, etc.)

63 A tense logic is a normal bimodal logic containing all instances of the schemata $\varphi \rightarrow \square \diamond^{-1} \varphi$ and $\varphi \rightarrow \square^{-1} \diamond \varphi$; the weakest such logic is called $\mathrm{K}_{t}$. Historically such logics were considered first by A. N. Prior with a temporal reading in mind, the points at which formulas are evaluated being thought of as representing temporal instants and the associated accessibility relation for $\square$ being thought of as the earlier than relation and for $\square^{-1}$ its converse; there is no essential connection with time and tense, however, since the accessibility relation in any frame has a perfectly good converse no less deserving of expression in modal terms than the original relation. 
satisfied, the second pair the property of being a $y$ such that for all $w, x, z$, the condition is satisfied, and so on.

With a view to showing that, unlike some of the above examples, the smallest normal monomodal logic, $\mathrm{K}$, can accommodate whetherdisjunction conservatively, we approach the issue via the currently envisaged tense logic, for which as an ad hoc label we will simply use S, rather than something more informative ( such as "K $\mathrm{K}_{t} \oplus\{G \varphi \rightarrow G H G \varphi\}$ "). Note that we have $\vdash_{\mathrm{s}}(G \varphi \vee G \psi) \leftrightarrow G(H G \varphi \vee H G \psi)$. (The $\leftarrow$-direction we have in $\mathrm{K}_{t}$ already, the $\rightarrow$ direction requiring the special (GE) axiom.) To get this to help with the problem of monomodal $\mathrm{K}$, we need an observation:

Lemma 7.6. $\mathrm{K}$ is determined by a class of frames satisfying the generalized equivalence condition.

Proof. K determined by the class of all frames satisfying "single predecessor condition" - i.e., frames $\langle W, R\rangle$ such that for all $v \in W$ there exists at most one $u \in W$ with Ruv, since the unravelling of any frame, as in [Sahlqvist, 1975], satisfies this condition, and $\mathrm{K}$ is determined by the class of all unravellings of frames. But the single predecessor condition implies the generalized equivalence condition, giving the desired result.

Thus all we need to do is capture the semantic force of the above disjunction $H G \varphi \vee H G \psi$, so that we can prefix a " $G$ " to it - though to emphasize that our language is now monomodal, we write $\square$ rather than $G$; since we envisage \# as a new connective, the phrase "normal extension" should have "normal" be understood consistently with this, i.e., as applying to a set of formulas (of the expanded language) containing all theorems of $\mathrm{K}$ and closed under Uniform Substitution, Modus Ponens and Necessitation (prefixing of $\square$, that is):

Theorem 7.7. The normal extension of $\mathrm{K}$ by the addition of a new binary connective \# for which, whatever formulas $\varphi$ and $\psi$ may be, $\square(\varphi \# \psi) \leftrightarrow(\square \varphi \vee \square \psi)$ is provable, is conservative.

Proof. Suppose that the envisaged extension of $\mathrm{K}$ proves a formula $\chi$ not containing any \#-subformulas, but not already provable in $\mathrm{K}$. Thus by the Lemma, $\chi$ is false at some point, $u$, say, in a model on a frame satisfying the generalized equivalence condition. Interpret formulas $\varphi \# \psi$ in this model, $\mathcal{M}=\langle W, R, V\rangle$, say, in according with the condition that for any point $x \in W$ : 
$\mathcal{M}, x \models \varphi \# \psi$ iff $\quad$ either (1): for all $y \in W$ such that $R y x$, for all $z \in W$, if $R y z$ then we have $\mathcal{M}, z \models \varphi$, or (2): for all $y \in W$ such that $R y x$, for all $z \in W$ if $R y z$, then we have $\mathcal{M}, z \models \psi$.

By the considerations about the tense-logical $\mathrm{S}$ above, all formulas of the form $\square(\varphi \# \psi) \leftrightarrow(\square \varphi \vee \square \psi)$ are true throughout this model while $\chi$ is false at $u$ (not being affected by this stipulation about \# since it contains no \#-subformulas), meaning that $\chi$ is not provable in $\mathrm{K}$ by the soundness of $\mathrm{K}$ w.r.t. the class of all frames.

Since this proof of Theorem 7.7 proceeded via a consideration of the basic tense logic $\mathrm{K}_{t}$, at this point a natural question arises as to the conservativity of the extension of $\mathrm{K}_{t}$ by whether-disjunction. As before, we write " $G$ " for " $\square$ " in this setting. In this case, we have a contrast with the monomodal case, the answer here being negative:

TheOREM 7.8. The normal extension of $\mathrm{K}_{t}$ by the addition of a new binary connective \# for which, whatever formulas $\varphi$ and $\psi$, may be, $G(\varphi \# \psi) \leftrightarrow(G \varphi \vee G \psi)$ is provable, is nonconservative.

Proof. We use the fact that $\vdash_{\mathrm{K}_{t}} G P G p \rightarrow G p$ while $\nvdash_{\mathrm{K}_{t}} G P(G p \vee$ $G q) \rightarrow(G p \vee G q)$. With \# behaving as described, we would be able to get from the first provable formula to the second unprovable one by substituting $p \# q$ for $p$ :

$$
G P G(p \# q) \rightarrow G(p \# q),
$$

and then unpack the two $G(p \# q)$ subformulas in accordance with the whether disjunction equivalence for \#, getting the otherwise unprovable (and \#-free) $G P(G p \vee G q) \rightarrow(G p \vee G q)$.

In case it is not clear that, as claimed in this proof, $\nvdash_{\mathrm{K}_{t}} G P(G p \vee$ $G q) \rightarrow(G p \vee G q)$, this can gleaned from the five-element frame depicted in Figure 1, though the caption on the diagram should be ignored for the moment - we return to it presently - and the broken arrows read as indicated that the nodes at their heads are not successors of those at their tails by the frame's accessibility relation (and the solid arrows indicating that here the head points are accessible to the tail points). Consider a model on this frame which makes $p$ true at just the point $y$, and $q$ true at just the point $z$. Then $G p \vee G q$ is not true at $w$, whereas $G P(G p \vee G q)$ is, since $P(G p \vee G q)$ is true at $y$ in view of $v$, where $G p$ is true, as well as at $z$, in view of $x$, where $G q$ is true. 


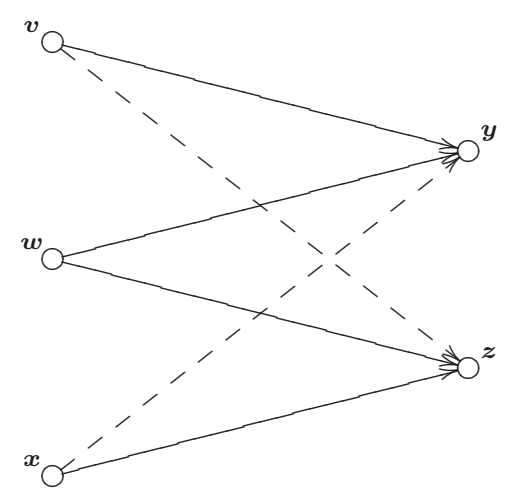

Figure 1: A Diagrammatic Representation of the Weak GE Condition

The actual caption under Figure 1 is suited for what the figure depicts directly as a universally quantified first-order condition on frames, which (as for such diagrams in [Humberstone, 2016]) is this: if the accessibility relation holds between points as indicated by the solid arrows then at it holds between at least one of the pairs of points linked by broken arrows. (The labelling of the nodes is of course redundant in the representation of such conditions, and was included only for its use in another capacity in the preceding paragraph.) The frame described in the preceding paragraph in order to invalidate the formula $G P(G p \vee G q) \rightarrow(G p \vee G q)$ violates this condition, but so would any larger frame containing points beyond those explicitly indicated in Figure 1. If we spell out the first-order condition the diagram conveys, we get something reminiscent of condition (GE), only weaker, namely the universal closure of the following:

$$
(R v y \wedge R w y \wedge R w z \wedge R x z) \rightarrow(R v z \vee R x y)
$$

A corresponding diagram for (GE) itself can be obtained by discarding the point $v$ and arrows appended thereto. ${ }^{64}$ The class of frames satisfying (weak GE) is tense-logically defined by either of the formulas

$$
\begin{gathered}
G(P G p \rightarrow q) \vee G(P G q \rightarrow p) \\
G((p \vee P G q) \wedge(P G p \vee q)) \rightarrow(G p \vee G q)
\end{gathered}
$$

and the normal extension of $\mathrm{K}_{t}$ by either of (7.1), (7.2), gives the logic determined by this class of frames. Though it is more cumbersome, we

${ }^{64}$ The (GE) diagram appears, in a different orientation, as Figure 5.6 at the top of p. 444 in [Humberstone, 2016]. 
include (7.2) here because of its similarity to a third, also somewhat cumbersome formula, obtained by some $\wedge / \vee$ distribution in the (scope of $G$ in the) antecedent, with subsequent simplifications:

$$
G((p \wedge q) \vee(P G p \vee P G q)) \rightarrow(G p \vee G q)
$$

This variant we can compare easily with a minor reformulation of the formula $G P(G p \vee G q) \rightarrow(G p \vee G q)$ figuring as our nonconservativity witness in the proof of Theorem 7.8; here we simply distribute the $P$ in the antecedent across the disjunction:

$$
G(P G p \vee P G q) \rightarrow(G p \vee G q)
$$

So we can see our (weak GE) defining formula (7.3) as the result of strengthening our nonconservativity example (7.4) by weakening its antecedent with the addition of a further disjunct (namely: $p \wedge q$ ) in the scope of the outer " $G$ " there. ((7.4) itself modally defines the class of frames $\langle W, R\rangle$ satisfying the condition:

$$
\forall w \in W \forall x, y \in R(w) \exists v \in R(w) \forall u \in R^{-1}(v) \text {. Rux } \wedge \text { Ruy. }
$$

and the canonical frame for $\mathrm{K}_{t} \oplus\{(7.3)\}$ can be shown to satisfy this condition.)

As usual with questions of conservative extension in logic, there are what might be called two degrees of conservativity. The weaker property being that the envisaged extension is conservative because while there is no connective in the language of the logic behaving as the extension in question would have it behave, a new connective can be added behaving in that way without this rendering provable anything free of that connective which was not formerly provable. The stronger property is that there is already a connective (not necessarily a primitive connective) behaving as the extension would have it behave, the extension is conservative because (to within notational changes) we do not need to consider a proper extension. Whether-disjunction is conservative over S4 (and its extensions) because here we can put $\varphi \# \psi=\square \varphi \vee \square \psi$ and we have $\square(\varphi \# \psi)$ and $\square \varphi \vee \square \psi$ provably equivalent. Evidently in the case of $\mathrm{K}$ itself there is no such endogenous candidate for \#, or else we could not get nonconservative effects in stronger normal modal logics, such as that recalled for $\mathrm{K}_{c}$ and $\mathrm{K} 4$ ! above.

Since there is a well-known contrast between the grammaticality of the perfectly acceptable " $a$ knows whether $\varphi$ " and the wildly unaccept- 
able " $a$ believes whether $\varphi$ ", one might hold out some hope via a connection between this contrast and the contrast in factivity between knowledge and belief, for the conservative extension of all normal $S \supseteq$ KT by whether-disjunction, and not just as in the $\mathbf{S} 4$ type cases just reviewed in which such a connective is endogenously available. We conclude this section by showing that whether-disjunction is not guaranteed, as the hope just articulated would have it, to be conservative over arbitrary extensions of KT. In fact we already have the materials to hand for a counterexample. We can first recycle our $\mathrm{K}_{t}$ example in a monomodal setting using KB as "the poor man's $\mathrm{K}_{t}$ ". That is, we simply restrict attention to symmetric frames so that we can write both " $G$ " and " $H$ " and " $G$ ", or indeed, for familiarity, as " $\square$ ". So rewritten the $\mathrm{K}_{t}$-unprovable formula figuring in the proof of Theorem 7.8 becomes:

$$
\square \diamond(\square p \vee \square q) \rightarrow(\square p \vee \square q) .
$$

(Of course, we could equally well write the antecedent as $\square(\diamond \square p \vee \diamond \square q)$, in the style of (7.4).) To confirm that this formula is not KB-provable, we show how to invalidate it on the symmetric frame depicted in Figure 2; as usual with undirected graphs, we omit what might otherwise be the expected arrowheads on our edges.

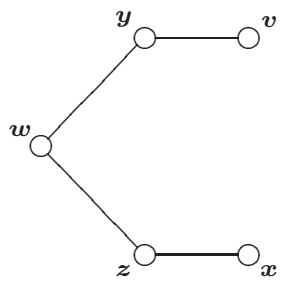

Figure 2: A Symmetric Frame Invalidating $\square \diamond(\square p \vee \square q) \rightarrow(\square p \vee \square q)$

The frame of Figure 2 could equally well have been drawn as five points in a line, but here they are laid out, and also labelled, in such a way as to recall Figure 1: if we forget about symmetry and points are accessible just to those linked to them and on their left, we would have a non-symmetric (indeed asymmetric) frame which would suffice for refuting the hypothesis that our implication was provable in $\mathrm{K}$. The $\mathrm{K}_{t}$ version of this which has $\diamond^{-1}$ (written as $P$ ) is what the frame of Figure 1 was used to invalidate, with $v$ and $x$ bent back to positions behind $x$ and $y$ to reflect the shift from $\diamond$ to $\diamond^{-1}$ with the convention that $R$-successors of a point appear to the right of that point them in the diagram. 
With $\langle W, R\rangle$ as depicted, let $V(p)=W \backslash\{z\}, V(q)=W \backslash\{y\} ; V$ can assign any subsets of $W$ to the remaining sentence letters. Then $\mathcal{M}=\langle W, R, V\rangle$ verifies the antecedent of our formula at $w$ since each of its two accessible points $y, z$ verify $\diamond(\square p \vee \square q)$, in $y$ 's case because of the $\square p$-verifying successor $v$ and in $z$ 's case because of the $\square q$-verifying successor $x$. But the consequent $\square p \vee \square q$ is false at $w$ in $\mathcal{M}$, because of $w$ 's $p$-falsifying successor $z$ and $q$-falsifying successor $y$. This shows, then, that whether-disjunction extends KB nonconservatively. (More generally, our formula is valid on exactly the frames satisfying a condition like $(\star)$ above, except that the " $R^{-1}(v)$ " in that condition is replaced by $R(v)$. Note that " $v$ " is a variable there, and not, as in the discussion based on Figure 2, the name of a particular frame element.)

But we promised to do that job for an extension of KT. As the foregoing discussion has perhaps suggested, the extension we have in mind is KTB. We need only tweak the example we have just worked through a little. We keep $W$ and $V$ as before, but we need to trade in $R$ for its reflexive closure - (i.e, $R \cup\{\langle u, u\rangle \mid u \in W\}$ ). Because of the way $V$ was defined, we still have the antecedent of our implication true at $w$, even though there is now an additional point accessible to $w$, namely $w$ itself, so we need to check that $\diamond(\square p \vee \square q)$ is true at $w$ in the new model, which it is since $\square p$ is true at $y$ in that model. (We could equally have used $z$ and $\square q$.)

What of KT itself? Example 7.2 showed that Uniform Substitution did not preserve validity over reflexive frames in the inquisitive semantics (with $(\mathrm{C} \square)$ in force), but the example does not immediately show that whether-disjunction is nonconservative over KT. Accordingly we leave this as an open question.

\section{Two Comments and an Update on Property Supervenience}

\section{First Comment: Excessively Restrictive Accounts}

For the sake of those particularly interested in formal aspects of property supervenience, this section elaborates on Remark 1.1 in the light of subsequent developments in our discussion, especially in Sections 1 and 2. The main comments address, first, the tendency of writers on supervenience to impose inappropriately strong demands on when this relation obtains, and second, the bifurcation of this concept into an intra-world and inter-world variant. In between these two are interposed some re- 
marks about what the relata of this relation (in either of the two variants just contrasted) are best taken to be, and after them an update looks at a newer line of enquiry: the fate of some striking twentieth-century arguments (mainly associated with Jaegwon Kim) to subsequent attention (mainly by Ralph Wedgwood) over the exact choice of modal logic for metaphysical necessity.

To address the first topic, we begin by recalling that the basic idea is that a property $P$ is supervenient on a set $\mathbb{Q}$ of properties if any two individuals agreeing in respect of each $Q \in \mathbb{Q}$ agree in respect of $P$. Despite the mainly discursive approach of the present section, some immediate qualification and elaboration of this definition we include in a numbered Remark to facilitate cross-referencing. (For a cursory reading of this section, omit such numbered Remarks - unless following up such a reference - as well as the passages labelled 'Digression' which go into more detail than such a reading requires.)

Remark 8.1. Here we treat property talk as a reification of what could be said in the formal mode by speaking of predicates, and in particular refrain from defining a property as a function from worlds to sets of objects (having the property in the world in question). If the latter are taken as properties sensu stricto, then the present notion is something like that of a pre-property in roughly the sense of [Bartsch, 1986]: functions from a suitable set of parameters to properties in the strict sense. Those parameters might again be possible worlds (giving 'property concepts'), or perhaps 'centered worlds' (as in [Chalmers, 2004]), or agents/subjects (for a first-person indexical account, as in [Dreier, 1990] and Section IV of [Dreier, 1992]) or moral codes, though of as linking normative predicates to the appropriate properties, as in [Gibbard, 1992] — with a similar line taken in [Dreier, 1992], esp. Section IV again - and more particularly [Gibbard, 2003]. (One might follow Blackburn - see the incidental discussion in [Dreier, 2015b] - in speaking, instead, of properties as the shadows of predicates, though this is not quite accurate for the present understanding of pre-properties, since the would-be predicates, in this or that language, need not exist.) [Gibbard, 2003] devotes a chapter (Chapter 6) to explaining how supervenience works in this setting, conceding that anyone insisting this is a relation among properties sensu stricto might prefer to call it quasi-supervenience. (On p. 32 of [Gibbard, 2003] we also have a sympathetic emendation of a point from G. E. Moore: "All properties are natural, but some concepts are non-naturalistic." In 
the same vein, Jackson [1998] locates his discussion of 'the supervenience of the ethical on the descriptive' to quote the subsection title, within a section called 'Ethical Properties are Descriptive Properties'. Broadly similar sentiments can be found in [Pigden, 2012] and other works by the same author there cited.) Another example arises with vagueness, if one thinks that some predicates are vague but no properties can be vague, while still wanting to say that being tall or being bald supervenes on height and hair distribution properties. (The latter example, from [Jackson, 1998, p. 22] has complications, there noted, from the weak/strong/global distinction not germane to the present about needing to take property talk more liberally than one might otherwise be inclined to; the former example is even worse, raising the issue of attributive adjectives.)

Returning to the definition of supervenience given before the above Remark, let us observe that it has as an immediate consequence the following: if $P \in \mathbb{Q}$, then $P$ is supervenient on $\mathbb{Q}$. In our discussion this surfaced in the claim that the relations $\vdash_{\mathcal{V}}^{\text {svc }}$ were consequence relations, since this involves inter alia the claim that if $\psi \in \Gamma$ then $\Gamma \vdash_{\mathcal{V}}^{\text {svc }} \psi$. In a much discussed early paper on the subject, [Blackburn, 1971], Simon Blackburn ventured the following definition (labelled $\left(\mathrm{S}_{1}\right)$ on p. 105, italics added here):

A property $M$ is supervenient upon properties $N_{1} \ldots N_{n}$ if $M$ is not identical with any of $N_{1} \ldots N_{n}$, nor with any truth-function of them, and it is logically impossible that a thing which is $M$ should cease to be $M$ without changing in respect of $N_{1} \ldots N_{n}$.

There are obvious problems with what follows the "and it is logically impossible" here, described in the following Digression, since our main concern is with precedes these words.

Digression on Sharing vs. Agreeing. The diachronic element is particularly out of place in the definition, which in this respect would be like defining an essential property of an individual to be a property the individual has and could not lose while continuing to exist. That should follow from the property's being essential but not be used to define the latter as there are many properties which are not essential to an individual - the individual could have existed without possessing them, that is - but are necessarily unloseable (e.g., the property of having been born in Detroit). And if one were, for whatever reason, going to take 
this diachronic route, ${ }^{65}$ one should not want just "a thing which is $M$ should cease to be $M$ without changing in respect..." but rather "thing should change in respect of $M$ without changing in respect..." since not only ceasing to have but also coming to have a property needs to be incompatible with an absence of change in respect of the properties on which its supervenience is under consideration. ${ }^{66}$ There is potentially a similar issue with a definition of weak supervenience of properties in a set $A$ on those in a set $B$ given at p. 47 of [Kim, 1984a] (and similarly at p. 158 of [Kim, 1984b]) thus: "necessarily for any $x$ and $y$ if $x$ and $y$ share all properties in $B$ then $x$ and $y$ share all properties in $A$ ". Since for $x$ and $y$ to share a property is for both of them to have the property, the quoted "share" should have been "agree w.r.t." or "be alike in respect of". This is only potentially a danger since when a set of properties involved talking of sharing is naturally interpreted as agreeing on each property in the set, much as with describing two people as sharing the same movie preferences. But one needs to notice, just for the record, that in this sense sharing the same properties in some set of properties is not the same as sharing each property in the set. (If we were talking about statements rather than properties, an ambiguity in talk of two worlds agreeing on $\varphi$ would need to be mentioned. This might mean agreement as to whether $\varphi$, as in Section 1 above as well as in [Humberstone, 2002], i.e., both verify or both falsify $\varphi$; but it might also mean agreement on its being the case that $\varphi$, i.e., both verify $\varphi$, as in [Groenendijk, 2009]. There is the same issue with the word "settled" as applied to statements, in [Ciardelli, 2016a, pp. 5, 47] and elsewhere, a statement's being settled is a matter of its being settled as true, whereas in [Humberstone, 1996c] this is called being affirmatively settled, and "settled" means affirmatively or negatively settled. ${ }^{67}$ More precisely, in [Ciardelli, 2016a] a statement is

${ }^{65}$ Which can be avoided using, for instance, a suggestion of Noonan's in note 1 of [1987].

66 Minor grammatical hygiene: the " $M$ ", " $N_{1}$ ", etc., here are introduced as names of properties so talk of being $M$ should strictly be replaced by talk of having $M$, etc. But the convenience of the double usage-nominal and predicative - of symbols for properties is too great to forgo and is exploited without apology below, for instance in the formulations (WS) and (SS).

67 Compare also the formulation " $\varphi$ 's truth-value settles $\psi$ 's everywhere in the model" in the last paragraph of Section 1 above. A similarly even-handed use of settled is made by Ciardelli when speaking not of statements but of questions; in particular, a polar question is settled relative to a set of worlds when it has the same answer in all of them. The term "supports" rather than "settles", which is used in 
settled in an information state, construed as a set of worlds, when it is true at the worlds it contains, and in [Humberstone, 1996c] a statement is $M$-settled ${ }^{+}$at a world when it is true at all worlds which are like that world in respect of subject matter $M, M$-settled ${ }^{-}$there if it is false at all worlds like it in respect of $M$, and $M$-settled at a world if it is either $M$-settled ${ }^{+}$- or $M$-settled ${ }^{-}$-settled at that world. The notion to being $M$-settled ${ }^{+}$at $w$, with "all" replaced by "some" in the definition, is called simply being true about $M$ in $w$ in [Yablo, 2014, p. 32] - though in fact Yablo writes " $m$ " rather than " $M$ ".)

Later (p. 106), Blackburn revises $\left(S_{1}\right)$ to a formulation $\left(S_{2}\right)$ which removes the diachronic element, but incorporates another change he has made to $\left(\mathrm{S}_{1}\right)$ in an interim formulation labelled simply $(\mathrm{S})$, namely the introduction of talk of things having the properties $N_{i}$ to the same degree (in which case they need to have $M$ to the same degree): but the usual understanding of 'property' in this connection is that of determinate rather than determinable property, making the relevant properties the degree-relativized forms of the magnitudes in question in the first place.

The sharing vs. agreeing issue also arises in connection with a putative notion of supervenience extracted from [Blackburn, 1984] in [Wright, 1985 , p. 315] which purports to be a binary relation between properties (not between classes of properties, or between classes of properties and individual properties - to which contrast we return in the Intermission below). Here is the definition (using Wright's notation for properties ${ }^{68}$ ): $\psi$ is defined to supervene on $\varphi$ iff
(i)
$\square(\exists x(\varphi x \wedge \psi x) \rightarrow \forall x(\varphi x \rightarrow \psi x))$
and
(ii) $\neg \square \forall x(\varphi x \rightarrow \psi x)$.

The vagueness of Blackburn's discussion makes it hard to be sure if this attribution is correct, but certainly we see in (ii) the tendency to overload the definition of supervenience with restrictive features which the present Comment is directed against. (The problem as it was set out in [Blackburn, 1971] was how we could make sense of the combination of, to use terminology introduced below in our 'Second Comment', intra-world supervenience with (ii) - or the failure of something called E-perhaps

Ciardelli's formal discussions - cf. Section 6 above - does not raise the difficulty, since for statements this is a matter of settling affirmatively.

68 As the review is typeset in Mind, the " $x$ " here appears incorrectly as " $\chi$ ". (Clearly Wright would not have used a letter for his individual variables from the same alphabetic range as the predicate letters come. The typesetter must have thought "Okay, so the special symbols here are all lower case Greek letters.") 
suggestive of "Entailment" - in [Blackburn, 1971]. This was supposed to be especially problematic for the moral realist. But with (ii) built into the definition it looks as though it is supervenience simpliciter that is problematic, so let us set (ii) aside and suppose that (i) itself captured supervenience-more specifically, intra-world supervenience.) This means that we are considering the relation between property $\psi$ and property $\varphi$ when $\square(\exists x(\varphi x \wedge \psi x) \rightarrow \forall x(\varphi x \rightarrow \psi x))$, which can conveniently be written in the equivalent form (as on p. 694 of [Humberstone, 1998a])

$$
\square(\forall x(\varphi x \rightarrow \psi x) \vee \forall x(\varphi x \rightarrow \neg \psi x)),
$$

which displays it as a condition of relative possessor-independence: among the things (in any given world) with property $\varphi, \psi$ is a possessorindependent property. So any two things sharing $\varphi$ will agree w.r.t. $\psi$. But for supervenience more was always wanted than this, namely that any two things agreeing w.r.t. $\varphi$ will agree w.r.t. $\psi$, and the present condition - whatever Blackburn's intentions may have been - says nothing of thing agreeing over $\varphi$ by both lacking it. (Nor would tacking part (ii) of the putative definition back on assist with this.) End of Digression.

Let us turn to what precedes the words "and it is logically impossible" in the passage quoted from [Blackburn, 1971]. In the first place there is the condition imposed in "if $M$ is not identical with any of $N_{1} \ldots N_{n}$ " which precisely excludes the case of a property being supervenient on any set containing that very property. Naturally one can define a piece of terminology as one sees fit, but given the intuitive idea of supervenience as "no difference here without a difference there" it seems unwise to exclude in the definition what one may regard as trivial cases in which this relation obtains, rather than to let the definition include these, and remark that the cases of interest for such-and-such discussion lie elsewhere. In the second place, it is especially unfortunate to exclude, perhaps from the same disallow-the-trivial-cases motivation, the possibility of truthfunctional combinations as Blackburn does, since that deprives one of making the observation that there can be no difference in respect of the property of being, for example, being square and not red, without a difference in respect of at least one of the properties being square, being red. (In our discussion above, this surfaced as the satisfaction the condition (2.1) by all Boolean connectives \#; it will arise again in an example from Oddie and Tichý under 'Second Comment' below.) 
A variation on this first comment applies also to a characterization by Keith Campbell of supervenience, quoted at the beginning of [Bacon, 1986]:

One feature is supervenient upon others if, while not following deductively from those others, it nevertheless cannot vary unless they do.

Bacon describes this as a clear definition, but even once it is explained (for reasons given in note 66) that it is modal rather than just temporal variation that is at issue, the unclarity introduced by "while" cannot go unremarked. Is Campbell trying to say that if possession of one property follows from the possession of others, it cannot be supervenient on them? Or is he just making a comment on supervenience as standardly understood, to the effect that even if possession of a property does not follow from the possession of others it still qualifies as supervenient (when there could be no difference in respect of that property without a difference in respect of at least one of the others)? The second option - while as even if - amounts to an obscure formulation of the definition, while the first option involves an unwanted constraint similar to that just found in Blackburn, in that it too would imply that if $P \in \mathbb{Q}$ then $P$ was not supervenient on $\mathbb{Q} .{ }^{69}$

The above quotation from [Bacon, 1986] of Campbell's words suggests incidentally the question of when something both follows deductively from and also supervenes on some other things - in the main case of current interest, the 'things' are properties, and in the sentential setting of earlier sections of the present paper they formulas, and in the latter case this question becomes the question of when, for some given $\mathcal{V}$ we have both

$$
\varphi_{1}, \ldots, \varphi_{n} \vdash_{\mathcal{V}}^{\text {svc }} \psi \quad \text { and also } \quad \varphi_{1}, \ldots, \varphi_{n} \vdash_{\mathcal{V}}^{\text {inf }} \psi
$$

Here we pause only to settle this question in the $n=1$ case, showing that, degenerate cases aside in which $\vdash_{\mathcal{V}}^{\text {svc }} \psi$ (i.e., $\vdash_{\mathcal{V}}^{\text {inf }} \psi$ or $\vdash_{\mathcal{V}}^{\text {inf }} \neg \psi$ ), this holds only when the formulas concerned are ('deductively') equivalent. The notation is as introduced for Proposition 2.1.

Proposition 8.2. Suppose that $\varphi \vdash_{\mathcal{V}}^{\text {svc }} \psi$ and also $\varphi \vdash_{\mathcal{V}}^{\text {inf }} \psi$, where the same conditions on the language and on $\mathcal{V}$ are satisfied as were imposed in Proposition 2.1. Then either $\varphi \longleftarrow_{\mathcal{V}}^{\inf } \psi$ or else $\vdash_{\mathcal{V}}^{\text {svc }} \psi$.

69 A further gratuitous restriction Blackburn imposes on supervenience is given by his 'limitation thesis' on p. 51 of [Blackburn, 1985], but we do not go into this here. 
Proof. Suppose that the background conditions for this result from Prop. 2.1 are satisfied, and that for $\varphi, \psi$, we have (1) $\varphi \vdash_{\mathcal{V}}^{\text {svc }} \psi$ and (2) $\varphi \vdash \mathcal{V}$ inf $\psi$, and in addition (3) $\nvdash \mathcal{V}$ svc $\psi$, with a view to showing that in that case $\varphi-\nVdash_{\mathcal{V}}^{\text {inf }} \psi$. By (1) and the general condition from Example 1.5 (i), we have $\psi \vdash \vdash_{\mathcal{V}}^{\text {svc }} \varphi$ or $\vdash_{\mathcal{V}}^{\text {svc }} \psi$, but this second option is ruled out by (3), so we have the first option, which in view of (1) again, means that we have $\varphi-\nVdash_{\mathcal{V}}^{\text {svc }} \psi$. Thus by Prop. 2.1, we have $\varphi-\nVdash_{\mathcal{V}}^{\text {inf }} \psi$ or $\varphi \dashv \nVdash_{\mathcal{V}}^{\text {inf }} \neg \psi$. Since the first alternative here is our desired conclusion, it suffices to rule out the second. The $\vdash$-direction of the second alternative, taken together with (2), gives the conclusion that $\vdash \mathcal{\mathcal { i n f }} \neg \varphi$. The $\dashv$-direction of the second alternative implies that $\neg \varphi \vdash \mathcal{V}$ inf $\psi$. Together these would allow us to conclude (by the 'cut' condition on consequence relations) that $\vdash \stackrel{\mathcal{V}}{\text { inf }} \psi$, contradicting (3); thus the first alternative obtains.

\section{Intermission: The Fundamental Relata}

Before turning to the intra-world/inter-world or weak/strong contrast, I would like to suggest that there is something misleading about thinking, as in (WS) and (SS) of that discussion, and very generally in the literature, of supervenience as being fundamentally a binary relation between sets or classes of properties. ${ }^{70}$ To make this point we need the following

70 Take this remark from [Kim, 1984b], for example: "It is also clear that supervenience is better thought of as a relation not between properties or terms taken singly but between sets or families of them." Certainly the latter is preferable to the former, but here we shall argue that something intermediate is more revealing still: the relation holds fundamentally between a single property and a class of properties, and only derivatively between a class of properties and a class of properties, namely when each property in the former class is itself supervenient on the latter class. Before saying this Kim has just made the valuable point - against some of the ways R. M. Hare has expressed himself at though supervenience is a just property of properties: "goodness is supervenient" etc. Hare explains what he has in mind with some examples (p. 2 of [Hare, 1984]): being a nice room is supervenient, in that one could not have two things - in his example two rooms - which were similar all other respects save that one was a nice room and the other was not, whereas one could have two rooms which were similar in all other respects save that one was duck-egg blue and the other was not, so this property is not supervenient. It would be an interesting exercise to see if one could filter out the obvious counterexamples to this proposal in a satisfactory way. What colour might this other room be-pink, perhaps? Then the rooms also differ in respect of whether or not they are pink. Then take the 'respects' as determinables rather than determinates, might be the reply. ("They differ only in respect of colour.") But how do we get each determinate property to have a unique determinable under which it falls, as is needed for "all other respects" to pick out the respects in question 
terminology. ${ }^{71}$ Suppose $R\left(X_{1}, \ldots, X_{n}\right)$ is an $n$-ary relation among sets. Let us call such a relation $\forall$-reducible in its $i^{\text {th }}$ position $(1 \leq i \leq n)$ when for all $X_{1}, \ldots, X_{n}$ we have

$$
R\left(X_{1}, \ldots, X_{n}\right) \Longleftrightarrow \forall x \in X_{i} R\left(X_{1}, \ldots, X_{i-1},\{x\}, X_{i+1}, \ldots, X_{n}\right) .
$$

In fact we might justifiably use the same terminology for the $i^{\text {th }}$ position even some or all of the other positions are not there for sets at all. The ternary relation $R(\mathcal{M}, X, \varphi)$ holding between a model $\mathcal{M}$, as conceived in Section 3 a subset $X$ of the universe of $\mathcal{M}$ and a formula $\varphi$ just in case $\mathcal{M}, X \Vdash \varphi$. The formulas $\varphi$ which are in the Hodges-Väänänen sense flat (as explained in Section 4), or truth-conditional in Ciardelli's terminology (recalled in Section 6), are then precisely those for which this ternary relation is $\forall$-reducible in its second position.

One should also allow for consideration of a corresponding notion of $R$ 's being $\exists$-reducible in the $i^{\text {th }}$ position - just replace " $\forall x$ " with " $\exists x$ " though that property is not of particular concern here. Because of the one-to-one correspondence between elements of a set and unit subsets thereof, a relation which is $\forall$-reducible (or for that matter, $\exists$-reducible) in a given position can be replaced by a relation in which individuals rather than sets figure in that position: the set-talk was only coding a plural version of a reference to individuals. Thus consider the relation $R_{C n}$ associated with a consequence operation $C n$ : for sets $\Gamma, \Delta$ of formulas, we define $R_{C n}(\Gamma, \Delta)$ just in case $\Delta \subseteq C n(\Gamma)$. This relation is $\forall$-reducible in its second position, and so is less misleadingly traded in for the corresponding relation between sets of formulas and individual formulas: this is just the consequence relation $\vdash_{C n}$ standardly associated with $C n$. On the other hand, the generalized or multiple conclusion consequence relations $\Vdash$ we had occasion to consider in Remark 1.3(i) are typically not $\forall$-reducible (or $\exists$-reducible either ${ }^{72}$ ) in their second

given only the determinate property mentioned? And suppose the room in question has a window in it: won't the other non-duck-egg-blue room also differ in respect of the property of being a duck-egg blue room with a window? "Then filter out the logical consequence of differing in the respect in question." "But being a duck-egg blue room with a window isn't a logical consequence of being a duck-egg blue room." And so on.

71 See pp. 105-109 of [Humberstone, 1992], for further discussion; note that the point of this intermission applies to strong and weak supervenience but not to global supervenience, mentioned in Remark 8.4 below.

72 In [Humberstone, 2011], consequence relations and generalized consequence relations which are $\exists$-reducible in their first position are called left-prime, while gen- 
position, and so really do require the set-theoretic ascent the notation suggests, rather than the notation simply serving as an abbreviation for something that could be said using consequence relations proper.

Although talk of consequence relations most directly suggests a motivation from inference-determination, in view of the reference just made to Remark 1.3(i), it will come as no surprise-picking up on Remark 1.3(ii) - to the reader, via the case of supervenience-determined consequence relations, despite the popularity of the claim that supervenience as it features in the philosophical discussions cited in the present sections is fundamentally a relation between classes of properties, here we suggest that this "fundamentally" is quite out of place. For classes $\mathbb{P}, \mathbb{Q}$ of properties the relation:

$\mathbb{P}$ is supervenient on $\mathbb{Q}$

is $\forall$-reducible in its first position, and is thus only derivatively a binary relation between classes of properties, being fundamentally a relation between individual properties and classes of properties. (This is not to deny that the binary relation between sets and the binary relation between sets of properties and individual properties are interdefinable, which of course they are. The point is that the ascent to the level of sets is gratuitous in the position of what is said to be supervenient. See the end of Remark 1.3(ii). Note that with the $\vdash^{\text {svc }}$ and $\Vdash^{\text {svc }}$ notation what is supervenient goes on the right rather than - as inset above - on the left. But the point there made is that it would be a pity to exploit multiplicity on the right with $\Vdash^{\text {svc }}$ to simply abbreviate a multitude of $\vdash^{\text {svc }}$-claims, rather than to say something irreducibly new - as with Gentzen, and indeed Carnap, in the case of $\Perp^{\text {inf }}$.)

This is all a little surprising since the familiar gloss on the what it is for $\mathbb{P}$ to supervene on $\mathbb{Q}$, "There can be no difference in respect of $\mathbb{P}$-properties without a difference in respect of $\mathbb{Q}$-properties," sounds as though it is giving a symmetrical treatment to the two classes of properties, running against the drift of our recent discussion to the effect that it is only in the $\mathbb{Q}$ case that we are really making use of the fact that the properties have been collected into a set. In fact, however, thisvia the $\mathcal{V} \sim \mathcal{V}$ considerations of Section 1 - is like describing the relation between sets $\Gamma$ and $\Delta$ of statements obtaining when there cannot be a falsehood among the formulas in $\Delta$ without there being a falsehood

eralized consequence relations which are $\exists$-reducible in their second position are called right-prime. 
among those in $\Gamma$. This amounts to saying that for each formula in $\Delta$, it cannot be false unless at least one formula in $\Gamma$ is false, making the relation involved $\forall$-reducible in the $\Delta$ position.

\section{Second Comment: Weak/Strong}

The second comment to be made relating the present discussion to some of the literature will require us to back up and note an unclarity in the definition of supervenience in play in the first comment above (the point of which holds on either resolution of the unclarity), in the condition that "any two individuals agreeing in respect of each $Q \in \mathbb{Q}$ agree in respect of $P$ ". Do we mean that the individuals agree in a given world in respect of each $Q \in \mathbb{Q}$ in that world, in which case they agree in respect of $P$ in that world? Or do we mean that the individuals in question can be found in perhaps different worlds and must agree in respect of $P$ in the worlds in question if they agree over each $Q \in \mathbb{Q}$ in the respective worlds? A simple (if artificial) example of the contrast between the former intraworld variety supervenience and the latter inter-world variety, showing that the former does not imply the latter is provided in [Oddie and Tichý, 1990, p. 260]; in this passage they use the terminology of weak and strong supervenience for intra-world and inter-world supervenience respectively:

$[\mathrm{C}]$ onsider the property, $P$, of being red or blue according as Socrates is or is not snubnosed. [[The intended sense is conveyed in a note in [Oddie and Tichý, 1990], defining $P x$ as $(R x \wedge S) \vee(B x \wedge \neg S)$ in which $S$ represents "Socrates is snubnosed". $\left.{ }^{73}\right]$ It is readily seen that $P$ weakly, but not strongly, supervenes on the base consisting of the properties red and blue. For consider any world $w$ in which objects $x$ and $y$ are alike as regards the two properties: for example, both are red. Then if Socrates is snubnosed in $w$, both $x$ and $y$ have $P$ in $w$, and otherwise neither does. Thus $P$ weakly supervenes on $\{$ red, blue $\}$. On the other hand, let Socrates be snubnosed in world $u$ but not in $v$, and let $x$ be red in both $u$ and $v$. Then $x$ has $P$ in $u$ but not in $v$. Thus $P$ fails to strongly supervene on $\{r e d$, blue $\}$.

The $P$ in play in this example is conspicuously an extrinsic property on any plausible way of drawing the intrinsic/extrinsic distinction, and given David Lewis's principle of recombination ( $\$ 1.8$ of [Lewis, 1986]), this is an essential feature of such examples, as is pointed out in [Moyer, 2008],

73 Oddie and Tichý actually write " $S s$ " rather than just " $S$ ". 
and provides, as Moyer notes, the missing link in Blackburn's 'mixed worlds' argument from (especially) p. 53 of [Blackburn, 1985]. This is the argument against the intelligibility of intra-world supervenience without inter-world supervenience (or any thing else which would require the truth of necessary implications from, in the case of special interest to Blackburn, non-moral to moral predication): if there are worlds where $\exists x\left(N^{*} x \wedge M x\right)$ is true and worlds where $\exists x\left(N^{*} x \wedge \neg M x\right)$ is true, where $N^{*}$ is a maximal non-moral property and $M$ an arbitrary moral property, what explains the non-existence of ('mixed') worlds in which

$$
\exists x\left(N^{*} x \wedge M x\right) \wedge \exists x\left(N^{*} x \wedge \neg M x\right)
$$

is true? Moyer reminds us that we frequently encounter cases in which something of the form (1) is true while the corresponding (2) is not namely when $\varphi x$ and $\psi x$ (respectively $N^{*} x \wedge M x$ and $N^{*} x \wedge \neg M x$ in the present case) involve extrinsic predications: ${ }^{74}$

$$
\begin{aligned}
& \diamond \exists x(\varphi x) \wedge \diamond \exists x(\psi x) \\
& \diamond(\exists x(\varphi x) \wedge \exists x(\psi x)) .
\end{aligned}
$$

This may not be much of a consolation to Blackburn, however, who emphasizes [Blackburn, 1985, p. 48] that the supervenience base for the moral case may contain "all kinds of relational truths about the subject, truths about other things, and so on," so a version restricting the base in this way may not be congenial to him. (In any case, for the record, the whole 'mixed worlds' mystery arises from ignoring the fact that intuitive support for intra-world supervenience in the moral case extends to the inter-world case, as Elliot [1987] observes.) Here we suppress qualms about identifying the extrinsic/intrinsic distinction among properties with the relational/non-relational distinction among pre-properties, to use a term from Remark 8.1; consult [Humberstone, 1996b] for a fuller discussion of this distinction, or [Weatherson and Marshall, 2014] for a quick description of the issue.

74 In fact Moyer thinks the inference goes through, mediated by the principle of recombination, not only when specifically intrinsic properties are involved, but properties from a broader class of what he calls local properties. However, the characterization of this broader class in [Moyer, 2008, p. 199f.] is not clear, and is said to yield the result that being a brother (i.e., being male and having a sibling) is a local property, whereas existing in a world in which there is a unicorn is not a local property - yet these properties, (unrestrictedly) existential relational properties in the terminology of [Humberstone, 1996b], seem to be on a par in respect of all the considerations adduced by Moyer. 
Remark 8.3. Another issue suppressed here is whether the characterizations of properties (or pre-properties) featuring in supervenience claims such as that of the moral on the non-moral - are themselves worldinvariant. For example the pressure, felt in [Humberstone, 1996c; Karmo, 1988], to think of the classification of a statement as moral or otherwise as in at least some cases contingent might be held to held to afflict the classification of predicates also. This leads to a relativization of that classification to worlds and individuals. For instance, taking the line developed in [Humberstone, 1996c], the predicate "Is comfortable or should be made comfortable" would be classified as moral qua predicate of Ursula in a world in which Ursula is uncomfortable, though not moral qua predicate of her sister Cora relative to that same world, in which Cora is comfortable, while relative another world in which both are comfortable, the same predicate might be held to be non-moral because its application to either sister follows from the correct and morally neutral description of the sisters as comfortable.

For examples less artificial than that supplied in the passage quoted from Oddie and Tichý above, and more philosophically interesting (while not being too controversial), of the divergence between weak and strong supervenience, see Section II of [Noonan, 1987]. ${ }^{75}$ A straightforward everyday example was given in [Jackson, 1998, p. 10]: the property of being among the tallest things there are (understood as having a height-perhaps shared with others - than which nothing else has a greater height). ${ }^{76}$

75 The above discussion uses properties sensu stricto as characterized in Remark 8.1, which was tailored to the usual 'quantified modal logic' approach according to which the same individuals can exist with different properties in different worlds; for a Lewis-style 'counterpart theory' approach in which individuals - at least those not existing in all worlds - exist in exactly one, properties are just sets of (possible) individuals and $P$ is inter-world supervenient on $\mathbb{Q}$ if any individuals alike in membership in each $Q \in \mathbb{Q}$ are alike in membership in $P$, while the intra-world version would replace "individuals" here with "world-mates".

76 Essentially the same example was later used on p. 130 of [Moyer, 2008], with one improvement - putting "people" for "things", thereby avoiding the mistake of thinking that the world comes with a determinate division into things - and one change in the opposite direction: forgetting that because of ties for height, being among the tallest in the sense just explained does not mean being the tallest. (Of course, the phrase "among the tallest" also has an everyday use in which it does not capture the intention of the example and introduces an unwanted vagueness, namely: the property of having as one's something at least close to a height not exceeded by 
Relatedly, [Hintikka, 1972] calls the contrast between (1) and (2) Leibniz's distinction and points out that the presence of relational vocabulary in the $\varphi$ and $\psi$ involved, the inference from (1) to (2) can fail, ${ }^{77}$ remarking:

Thus Leibniz's distinction is without difference as long as relational concepts are not employed. This is a striking result in view of the often repeated claim that Leibniz wanted to dispense with relations in the last analysis, and to reduce them to non-relational concepts.

This naturally invites the reading that (1) and (2) are equivalent as long as the open formulas represented by $\varphi x$ and $\psi x$ are constructed using only monadic predicates - or, demodalizing the claim (as well as abstracting from any particular interpretation of the non-logical vocabulary): that if $\exists x(\varphi x)$ and $\exists x(\psi x)$ are both satisfiable formulas of monadic predicate logic without identity, then their conjunction is also satisfiable. But, Hintikka's confident tone notwithstanding, this is obviously not right: with monadic predicate letters $F$ and $G$, take $\varphi x$ as $F x$ and $\psi x$ as $G x \wedge \neg \exists x(F x)$. (So Leibniz's distinction would not be a distinction without a difference "as long as relational concepts are not employed.") This is no threat to the principle of recombination, however, since-now thinking of $F$ and $G$ as interpreted predicates, even if each expresses an intrinsic property, $\psi x$ (typically) will not.

We return to the contrast between intra-world and inter-world supervenience (alluded to at the end of Section 2); this contrast has always been part of the conceptual folklore of the subject, ${ }^{78}$ but tended to be replaced by - or simplified to - a contrast between weak and strong supervenience introduced by Jaegwon Kim - see for example [Kim, 1984a,b]; the crisp formulations (WS) and (SS) below of what Kim has in mind are taken from [Dreier, 1992, p. 14].

anything/anyone else's. In this sense, one can be among the tallest and still be shorter than someone else.)

77 Hintikka in fact uses the letters $A$ and $B$ here (p. 159f., or p. 261 of the reprint listed in our bibliography) for the present $\varphi$ and $\psi$. As an example of the failed inference he gives the transition from the joint unsatisfiability of "There is someone who is everybody's master" and "There is someone who is nobody's slave" to the satisfiability of their conjunction. For an example free of the complication this raises over the irreflexivity of the master of relation, consider "There is a building taller than every tree" and "There is a tree taller than every building".

78 The present author recalls being introduced to the distinction by André Gallois in conversation in the mid-1970s, for example. 
Remark 8.4. As mentioned in note 66 these formulations do not quite make sense because of a double use of the property variables - now in name position (" $Q \in \mathbb{Q}$ ") and now in predicate position (" $Q x ")$ - but the meaning is clear and the gain in brevity is welcome. An equivalent version of the contrast between (WS) and (SS) appears as that between (PWS) and (PSS) in [Divers, 1996]. Of course one could use Kim's weak/strong terminology for the original intra-world/inter-world distinction without buying into Kim's way of explaining the terminology. This is what is done for instance in [Oddie and Tichý, 1990], in the valuable survey [McLaughlin, 1995, p. 24] and in Leuenberger [2008], for instance. It should be mentioned that there are also numerous further variations on these themes, especially versions of global supervenience, which we do not go into detail on. Roughly, a global supervenience claim is the claim that any worlds different in such-and-such respect also differ in so-and-so respect, the most common form being that no any worlds differing in respect of the distribution of these properties without differing in respect of the distribution of those properties. For present purposes, because this is just special case of the general idea, which happens to involve (the distribution of) properties of individuals, we are not thinking of global supervenience as a case of property supervenience. (Note also that agreement in respect of the distribution of a property is not a bipartite equivalence relation like those considered in the discussion before Remark 1.7(i).) Several precisifications of this idea can be found in the literature, for example in [Kim, 1984b; Leuenberger, 2009; Shagrir, 2013; Steinberg, 2014]; see also [McLaughlin, 1995; Post, 1995; Stalnaker, 2003], and the discussions and further references provided by Bennett [2004]; Moyer [2008]; Paull and Sider [1992]; Williamson [2001]. An early study of some supervenience conditions (again not reviewed here) in the model theory of deontic logic can be found in [Rabinowicz, 1979], where, as in R. M. Hare's meta-ethical discussions (beginning with Hare [1952]), supervenience is very much an aspect of universalizability, the main subject of [Rabinowicz, 1979]. By contrast there appear several notions of supervenience of $P$ on $\mathbb{Q}$ in the Appendix (as well as the main body) of [McFetridge, 1985] likely to appeal to those with more haecceitist tendencies, such as one he calls $\left(\mathrm{XXWW}^{\prime}\right)$ : if any individual has precisely the same $\mathbb{Q}$ properties in any two worlds - "XX" in the label because we consider the same individual twice over. "WW'" because we allow the world (relative to which that individual is considered) to vary then that that individual must have the property $P$ in both worlds or in 
neither. (A version of this condition had appeared in the preceding year as (NC) on pp. 376 and 379 of [Klagge, 1984].)

$$
\begin{gathered}
\square \forall P \in \mathbb{P} \forall x(P x \rightarrow \exists Q \in \mathbb{Q}(Q x \wedge \forall y(Q y \rightarrow P y))) \\
\square \forall P \in \mathbb{P} \forall x(P x \rightarrow \exists Q \in \mathbb{Q}(Q x \wedge \square \forall y(Q y \rightarrow P y)))
\end{gathered}
$$

(WS) defines what it is for the set of properties $\mathbb{P}$ to supervene weakly on the set (of properties) $\mathbb{Q}$, and (SS), what is for $\mathbb{P}$ to supervene strongly on $\mathbb{Q}$. The question of whether we need to have an occurrence of $\square$ within the scope of another such occurrence to capture the force of (SS) will be touched on in the Digression on Klagge, below, as well as in the later 'update' subsection on dethroning S5. (An early appearance of a formulation in the style of (SS), emphasizing the need for the double appearance of $\square$, can be found in [McGinn, 1980]; it is explicitly quoted at the end of [McFetridge, 1985], where it is said to embody a 'principle of dependence' by contrast with the principles of (conditional) coincidence principally under discussion there, a terminology noted in Remark 1.1. Note, however, that this may still not capture the strong - and presumably hyperintensional - 'resultance' notion of dependence distinguished in Remark 1.1 from supervenience, as is observed in [Grimes, 1988, p. 155]; a similar point applies to some of what is called dependence in [Yoshimi, 2007].)

(WS) and (SS) manage to capture the notions of intra-world and inter-world supervenience when asking whether $P$ is supervenient on $\mathbb{Q}$ if we insist that $\mathbb{Q}$ (the 'supervenience base') is itself closed under Boolean operations - negation, conjunction and disjunction, including infinitary generalizations of these last two. In that case, for any world there is in $\mathbb{Q}$ for each individual - represented by the " $x$ " in the above formulations and $\mathbb{P}$-property $P$ possessed by that individual in that world, a maximally specific property possessed by the individual (a witness for the " $\exists Q$ " quantifier) implying for every $Q \in \mathbb{Q}$ either $Q$ or its negation (or complement), with all individuals in that (for (WS)) or any (for (SS)) world. ${ }^{79}$

79 An assumption along these lines is incorporated into a second pass by Blackburn over the terrain of [Blackburn, 1971] in [Blackburn, 1985], but the discussion there mixes up pure supervenience-related considerations with concerns about dependence or resultance in the sense of Remark 1.1 above. In fact the same can be said for the introductory discussion in [Kim, 1984b], too. Blackburn cleaned up his treatment in [1985] where there appears a binary connective $U$, with $\varphi U \psi$ having 
There are perhaps two reasons for the favourable reception Kim's strong supervenience, in particular, has had as an articulation of interworld supervenience, one of them being the background 'Boolean closure' assumption just mentioned has generally been found plausible for the main supervenience claims of interest to philosophers (supervenience of moral, aesthetic, and psychological properties on non-moral, nonaesthetic, or non-psychological properties respectively). ${ }^{80}$ Another reason is that there is a straightforward definition - namely (SS) - using the standard apparatus of quantified modal logic of strong supervenience though not of inter-world supervenience in the absence of Kim's special assumptions about the supervenience base. As Horgan [1993, p. 567] puts it: "If we attend carefully to the way modal expressions operate in discourse about supervenience, we find something happening that is not easily and directly expressible using the sentential modal operators of modal logic, viz. the transworldly comparison of individuals." The explanation of inter-world supervenience above used possible worlds vocabulary rather than modal vocabulary but the point stands: How do we say that $x$ and $y$ agree on the properties they have in (perhaps) different

the intended reading "its being the case that $\varphi$ underlies its being the case that $\psi$ ", removing this feature, for the presentation in pp. 182-185 of [Blackburn, 1984] (which I am assuming was written later, though the publication date is earlier). The feature in question persists in the work of some commentators on Blackburn, such as Elliot [1987], Brueckner [2002], and Shafer-Landau [1994]: see the "in virtue of" talk at p. 152 in this last reference.

80 Acquiescence has not been universal, however: see for example [Post, 1984, esp. p. 165], [Hellman, 1985], end of note 3 (p. 609), [Van Cleve, 1990, esp. p. 229], and [Lewis, 1985, p. 166f.]. Special attention is paid to issues arising over the use of infinitary forms of conjunctions and disjunction in this connection, in [Glanzberg, 2001] and [Bader, 2012], and is raised (with various other qualms) in note 11 of [Sobel, 2001], though Post was especially worried about the closure of the class of physical properties under plain old negation. An interesting suggestion in response to those worries is made on p. 62 of [Klagge, 1995]. Many are similarly dubious about whether the class of moral properties is closed under negation; for example, Heil [1995, p. 162] suggests that there are worlds in which no moral properties are instantiated - perhaps because devoid of agents. (See also pp. 38-41 of [Chalmers, 1996] for somewhat analogous issues in the philosophy of mind.) This would make not being praiseworthy, the negation (or complement) of a moral property, not itself count as a moral property. One encounters a similar thought that in the objection to standard deontic logic's equating permissibility of $a$ 's $\varphi$-ing with the absence of a requirement that $a$ should not $\varphi$, since if $a$ is the wrong kind of thing to fall under such requirements, the permissibility statement does not seem to be true. See the top paragraph of p. 252 of [Humberstone, 2016] for this and related issues. 
worlds? This problem is not as acute in such cases as transworld height comparisons ("Jane is taller than she would have been if her family had been less wealthy") which notoriously invite ontological inflation (adding heights, in the present case), because the relation of agreement in respect of a given property is a monadically representable binary relation, so there is no dyadic relational atomic sentence to be reformulated: which just need " $\leftrightarrow$ " between monadic predications. (See Chapter 6 of [Humberstone, 2016] for further explanation and background.) But Horgan is right to say that the idea is not "easily and directly expressible" using modal operators. ${ }^{81}$

Here is an offering in this direction from p. 95 of [Teller, 1985], where it appears as "(1)", though I have adjusted the notation somewhat, including omitting the " $\forall S$ " that would come after the " $\forall x$ ", since this corresponds to nothing in Teller's gloss (below):

$$
\begin{aligned}
\square \forall x\left[S x \rightarrow \exists \mathbb { P } _ { 0 } \subseteq \mathbb { P } \left(\forall P \in \mathbb{P}\left(P x \leftrightarrow P \in \mathbb{P}_{0}\right) \wedge\right.\right. \\
\left.\left.\square \forall y\left[\forall P \in \mathbb{P}\left(P y \leftrightarrow P \in \mathbb{P}_{0}\right) \rightarrow S y\right]\right)\right]
\end{aligned}
$$

Continuing with the adjusted notation - which includes changing Teller's " $y$ " to " $\mathbb{P}_{0}$ ", and his " $x$ " to " $y$ ", as well as making the $P \in \mathbb{P}$ explicit rather than conveyed by the choice of the letter " $P$ " 82 - we have the following gloss in [Teller, 1985]:

To help read (1) it says intuitively that for any $x$ in any possible world, if $x$ has property $S$, there is a set of properties, $\mathbb{P}_{0}$, constituting exactly the $\mathbb{P}$-properties which $x$ has, and for any $y$ in any possible world, if $y$ likewise has exactly the $\mathbb{P}$-properties in set $\mathbb{P}_{0}$, then $y$ also has property $S$.

From the gloss (or from the presence of the internal $\square$ in the symbolic formulation), we can see that this is intended as a modal formulation of inter-world supervenience, which does not (as (SS) does) require that there are maximal properties among those (collected into the set $\mathbb{P}$ ) on which the property $S$ is said to supervene, so there is no Kim-style

81 Likewise [McFetridge, 1985, p. 249]: "Once we have the explicit apparatus of possible worlds variables it is very easy to formulate supervenience claims that involve cross-world comparisons. (Such claims can be formulated without this apparatus but not nearly so perspicuously.)"

82 The reason for this is that the usual setting for many-sorted formulations is an assumption that the different sorts are disjoint, a presumption we do not want for the present case, in which it is properties of individuals which would be sorted, rather than individuals. For example, we want to allow $S \in \mathbb{P}$. 
(infinitary) Boolean closure assumption required (as a condition on $\mathbb{P}$ ). One might wonder if there is a less higher-order modal version available, avoiding the " $\exists \mathbb{P}_{0}$ " part.

Digression on Klagge. Klagge [1984, p. 379] offers something of a variation on (the historically later) (SS) without even the existential quantifier over properties. In order to quote his comments without too much adaptation, his symbolism for properties will be followed, and since what is of particular interest in [Klagge, 1984] is the supervenience of the moral on the natural, $M$ and $N$ are chosen as suggestive letters, except that rather than " $N$ " Klagge will use $\bar{N}$ with this explanation: "let the variable $\bar{N}$ range over maximally consistent, i.e., complete, sets of natural properties. (I have overscored the ' $N$ ' so that it is not confused with a variable that ranges over single properties, as the " $M$ " does.)" 83 The strong supervenience condition (SSS) offered by Klagge then reads as follows, sharing with (SS) above the occurrence of $\square$ within the scope of $\square$ :

$$
\square \forall x \forall M \forall \bar{N}[(M x \wedge \bar{N} x) \rightarrow \square \forall y(\bar{N} y \rightarrow M y)]
$$

A compact formulation indeed, though not one entirely explained by the preliminary remarks just quoted, which says that $\bar{N}$ ranges over (certain) sets of properties: yet here it is being used in predicate position, so some single property must be involved as the property being predicated presumably a maximal $N$-type property rather than an arbitrary such property (for which Blackburn's discussion has " $N^{*}$ "). An alternative would be that, as a name (see note $66, \bar{N}$ refers to a maximal set of properties $^{84}$ and as a predicate it predicates the higher order (but first level) property of having all the properties in that set. Or again that the predicated property is the intersection/conjunction of all the properties in the set. However this issue is resolved, it is hard to see proposing (SSS) as avoiding's Boolean closure assumption, smuggling it into the notation instead of using an existential quantifier as in Dreier's (SS) for which it is the provision of a witness that will require exploiting that assumption.

An interesting feature of (SS) and (SSS), which arises whether the two occurrences of $\square$ are left as they are, or notationally distinguished

83 The typesetters have not been kind to Klagge, here, with occasional over- and underscored characters both appearing on p. 379 as printed; evidently they did not recognise the convention of using underlining to indicate setting in italics.

${ }^{84}$ Consistency here is not really something to worry about, since we have the later antecedent " $M x \wedge \bar{N} x$ ". 
with a view to seeing different notions of necessity involved at the two positions (see the discussion preceding Remark 8.6 below) - for which reason we explain the issue on the former understanding - is that they involve a certain presumption as to the strength of the modal logic presumed to govern $\square$. To bring this out suppose that one wanted to write in tense-logical terms a principle that said that at any later time, if there is something which is $M$ and $\bar{N}$, then at all later times whatever is $\bar{N}$ will be $M$. One does not get this as the interpretation of (SSS) if one reads the $\square$ as the usual strong future tense operator ( $G$ in Prior's notation) because the first occurrence of this operator takes us to arbitrary later times and then second, being in its scope, then takes us on to times later than those times, rather than to all times later than the present. So the principle would not be counterexampled by cases in which there are future times at which are things which are $\bar{N}$ and not $M$ as well as future times at which there are things which $\bar{N}$ and $M$, as long as all the former times precede all the latter times. (Think of time as linearly ordered for this example.) One is naturally inclined to reach for an operator "Now" which can be placed before the inner occurrence of the $\square$ so that it is interpreted as taking us to arbitrary moments later than the original time of evaluation rather than to moments later than whatever time we are currently considering. For the motivating (alethic) modal example here, one could have similar recourse to a corresponding use of the operator "Actually" - which will figure briefly below but is not needed for solving the present problem, at least if we assuming that the universal property quantifiers commute with $\square$. Since in (SSS) they are sortally restricted, there are really two assumptions involved here: the first-implausible enough perhaps - is that the same properties exist at all worlds, and the second is that there status as natural or moral or whatever taxonomy the supervenience thesis under consideration is couched in terms of, is a noncontingent matter: something Remark 8.3 mentioned would be assumed for simplicity. Whatever their plausibility, it is of interest that if we have such a 'Barcan + converse Barcan' formula for the property quantifiers, we can move the latter to the front of (SSS), giving

$$
\forall M \forall \bar{N} \square \forall x[(M x \wedge \bar{N} x) \rightarrow \square \forall y(\bar{N} y \rightarrow M y)]
$$

Next, noticing that the " $\forall x$ " binds no variable in the consequent of the conditional which makes up its scope, we can do some more rewriting:

$$
\forall M \forall \bar{N} \square[\exists x(M x \wedge \bar{N} x) \rightarrow \square \forall y(\bar{N} y \rightarrow M y)]
$$


Now, discarding the initial property quantifiers and the quantificational details of what is in the scope of the outer box, we can consider the issue at the level of propositional modal logic, discerning in $\left(\mathrm{SSS}^{\prime \prime}\right)$ the pattern $\square(\varphi \rightarrow \square \psi)$, so it remains only to observe that among the normal modal logics, those in which the simplifying schema

$$
\square(\varphi \rightarrow \square \psi) \leftrightarrow(\diamond \varphi \rightarrow \square \psi)
$$

is provable are precisely the extensions of the logic $\mathrm{K} 45 .{ }^{85}$ In fact, the smallest normal modal logics containing (all instances of) the forward and backward halves of this biconditional schema are respectively $\mathrm{K} 5$ and K4. This means that in S5 in particular, we can effect this simplification and finally massage (SSS) into an equivalent modally first-degree form:

$$
\forall M \forall \bar{N}[\diamond \exists x(M x \wedge \bar{N} x) \rightarrow \square \forall y(\bar{N} y \rightarrow M y)]
$$

The interested reader is left to investigate the prospects for reducing modal degree in the case of the variegated forms of (SSS) in which the outer and inner occurrences of $\square$ are replaced by occurrences of different operators $-\square_{1}$ and $\square_{2}$, say - in the manner suggested by (especially) Dreier's discussion, as well as in similarly tackling Dreier's own formulation (SS), variegated or otherwise. In their unvariegated form, the contrast between principles like (SS) and (SSS), in which there is no modal embedding, on the one hand, and ( $\left.\mathrm{SSS}^{\prime \prime}\right)$ in which there is, on the other, raises a question about what might happen if the discussion were conducted against the backdrop of a modal logic in which the two forms were not equivalent. This is the subject of the 'Update' at the end of this section, on 'dethroning S5'. End of Digression.

85 Here we use the Lemmon-Segerberg-Chellas nomenclature, as explained for instance in [Humberstone, 2016], at p. 102 of which the special case of the schema inset above, in which $\psi$ is $\varphi$, is noted not to be S4-provable, its left and right hand sides giving two of the three S4-distinguishable notions of noncontingency (for $\varphi$ ) discussed there. The semantic significance of $\mathrm{K} 45$ is that it and all its normal extensions are determined by (= sound and complete w.r.t.) classes of models in which the points accessible to any given point are the same as the set of points accessible to any other point, so that we don't have to 'back up' with an actuality operator or any other such device (see [Humberstone, 2004a] for some alternatives) to go forward to other point accessible to the original point of evaluation - they will just coincide with the currently accessible points. (The inset equivalence preceding this note is a modal version of the equivalence that allowed us to trade in the outer " $\forall x$ " in $\left(\mathrm{SSS}^{\prime}\right)$ for the inner " $\exists$ " in $\left(\mathrm{SSS}^{\prime \prime}\right)$.) 
With the idea of avoiding the " $\exists \mathbb{P}_{0}$ " in mind - without "smuggling it in" via notational conventions, as in the above Digression - here we present a (none too attractive) formulation using the two-dimensional modal language explained in [Davies and Humberstone, 1980], q.v. for the semantics (which for simplicity assumes a universal accessibility relation and constant domains for the quantifiers), as a formulation of what it is for $P$ to be inter-world supervenient on a set $\mathbb{Q}$ of properties; read $\square, \mathcal{F}$, and $\mathcal{A}$, respectively, as necessarily, fixedly, and actually:

$$
\forall x \forall y \mathcal{F} \square[\forall Q \in \mathbb{Q}(Q x \leftrightarrow \mathcal{A}(Q y)) \rightarrow(P x \leftrightarrow \mathcal{A}(P y))]
$$

Note that we could equally well put the actually operators on the $x$ subformulas instead of the $y$-subformulas. Of course we are still quantifying over properties, but not over sets of properties, as in Teller's formulation. ${ }^{86}$

Remark 8.5. If the " $\mathcal{F}$ " is omitted from the formulation above, what we have is a modal version of the possible-worlds condition called (XYWA) on p. 257 of [McFetridge, 1985], an interesting cross-world supervenience condition falling short of unrestricted inter-world supervenience. The remark just made about equivalently re-locating the actuality operators corresponds to McFetridge's description (p. 257) of what in his nomenclature would be (XYAW) as a trivial variant of (XYWA). (For the notation, see Remark 8.4 above; here "A" is added to the W-slots specifically for the actual world.) In fact he explicitly uses (XXWA) and (XXAW) as examples of such trivial variation, where the doubled variable indicates that while these conditions cross worlds - actual-toarbitrary, rather than arbitrary-to-arbitrary, as with traditional interworld supervenience - they are not 'cross-individual' principles. Klagge [1987] takes issue with the intuitive plausibility of these supervenience principles, suggesting (p. 314) that McFetridge "has committed the fallacy of composition. From the admitted fact that (XXWA) is a condition on the moral judgments made by each person, it does not follow that it is a condition on the modal judgements of all people taken together or on moral truth." Of two formulations separated by "or" here, only the first has anything to do with a fallacy of composition, while only the second -

86 The trade-off between higher-order quantification (or equivalent) and the use of an "actually" operator is independently familiar; see, for example, the inset formula on p. 100 of [Humberstone, 1982]. It should be noted that the intended application of this language differs considerably from the use to which it has been to put above. 
with its contentious deployment of the notion of truth-bears on the issue; at any rate that issue concerns the content of the supervenience principles rather than facts about adherence, individual or collective, to them. (A sentiment similar to that expressed by Klagge here can also be found in note 17 of [Horgan and Timmons, 1992].) Klagge continues, rather surprisingly in view of his [1984] (see the end of Remark 8.4 above): "Possible-worlds formulations, do not preserve the relativization of this condition to perspectives considered distributively. They either treat perspectives collectively or drop reference to perspectives." When reference is dropped to a parameter, the usual convention in modeltheoretic semantics is that it is taken as fixed for the discussion; if on the other hand one is using the possible worlds vocabulary to make what would otherwise be modal claims, and the parameter is perspectival, it is not just held fixed for the discussion but taken as representing one's own perspective. (And when non-actual worlds are considered, they are to be considered from one's actual perspective - a feature stressed in [Elliot, 1987, p.136].) In any case what Klagge is calling perspectives can be explicitly introduced if desired: examples include the treatments of [Gibbard, 1992; Karmo, 1988].

The $\mathcal{F}$ formulation (before Remark 8.5) above is again is quite a mouthful, so what reason might one have for preferring not to make Kim's Boolean closure assumption, making available, as it does, the simple (WS) and (SS) style formulations? In fact this is just a straightforward replay of the considerations at the end of the opening paragraph of this section, but to conclude our second comment, let us see Oddie and Tichý making the relevant point at p. 260f. of [1990]:87

The motivation behind this [Boolean closure] requirement is not entirely obvious. It would seem natural to say, for example, that the property

87 Oddie and Tichý are mainly concerned in this paper with a non-Boolean closure condition called 're-splicing' explicitly proposed in [Bacon, 1986]. Sometimes such conditions are smuggled into the discussion much less explicitly. For example Klagge [1995, p. 67]: "In particular it seems that if $P$ is a property, then there is also a property $P^{\sharp}$ that holds of an object just in case $P$ holds of the object and there is another object in the world (formally $P^{\sharp} x \equiv[P x \&(\exists y) y \neq x]$ ). (Intuitively, $P^{\sharp}$ is a nonintrinsic subvening property - i.e., it is a relational property.)" Of course, for any property $P, P^{\sharp}$, so defined, is a perfectly good property, but that doesn't mean that every candidate supervenience base should be closed under the operation that takes us from properties $P$ to the corresponding $P^{\sharp}$. For example, we might be considering what was supervenient on the class of intrinsic physical properties. 
red or blue supervenes on the base $\{$ red, blue $\}$, for an individual's possession or otherwise of that property is obviously determined by its possession or otherwise of redness and blueness. Once it is required that a supervenience base be Boolean-closed we can say no such thing, for $\{$ red, blue $\}$ does not count as a supervenience base any more.

A 'live' example from the literature would be David Lewis's idea that intrinsic properties are those which are supervenient on the class of perfectly natural properties (see for instance [Lewis, 1983b, esp. p. 356] and also [Langton and Lewis, 1998]). In this case, the class of perfectly natural properties for Lewis is definitely not closed under negation or disjunction, but is taken to be a supervenience base for the intrinsic properties. $^{88}$

Digression. Kim's original formulation at p. 152 of [Kim, 1978], a paper he later became dissatisfied with for other reasons (see [Kim, 1984b], note 17), did not have this effect. There he explained what it was for a set $M$ of properties to be supervenient on a set $N$ of properties when indiscernibility w.r.t. the (Boolean - though we may as well take this, as in Kim's later work - as the infinitary Boolean) closure of $N$ implied indiscernibility (alias agreement) w.r.t. the closure of $M$ : so the supervenience base was not itself required to satisfy the closure condition. But note that the references to the Boolean closure of these sets are completely redundant and dropping them simply gives the standard definition of supervenience (in a formulation which is neutral over the intra-world/inter-world contrast). However, the currently envisaged way of handling the closure issue comes into its own when we consider Kim's formulation of his main observation in [Kim, 1978, p. 153]), here given

88 In a paper devoted to the extrinsic/intrinsic distinction, [Lewis, 1983a], published in the same year as [Lewis, 1983b], Lewis does not use the naturalness idea at all. He says that we can define intrinsicness in terms of duplication - the intrinsic properties being those on which duplicates must agree, and we can define duplication in terms of intrinsic properties, the former consisting in agreement in respect of the latter, though of course we would be involved in a ("tight little") circle if we tried to accept both as definitions. The new plan has us take for granted the perfectly natural properties and define duplication as agreement in respect of them and then intrinsicness as properties on which any duplicates must agree. (This illustrates the fact that if $\mathbb{Q}^{*}$ is the Boolean closure of $\mathbb{Q}$ then the properties supervenient on $\mathbb{Q}$ are precisely the properties supervenient on $\mathbb{Q}^{*}$.) For developments in thinking about intrinsic and extrinsic properties immediately subsequent to those cited here, see [Lewis, 2001] and [Weatherson, 2001]; a recurrently updated overview of the area is provided by Weatherson and Marshall [2014]. 
in an intra-world version: if $M$ is supervenient on $N$ then (in any world) each instantiated property in $M$ is coextensive with some Boolean combination of properties in $N .{ }^{89}$ This can be thought of (anachronistically) as a response to some of the qualms alluded to in note 80 by those worried by whether negations or disjunctions of (e.g.) physical properties are themselves physical. One could concede that they are not and still have something of interest to say in the claim that all psychological properties are coextensive with Boolean combinations of physical properties. On the other hand, some of the interest of such claim (or the analogous claim in the moral/natural case), since as [Teller, 1984] the properties arrived at by taking Boolean combinations of physical properties are unlike physical properties proper in that sharing such properties is no marker of physical similarity. End of Digression.

Oddie and Tichý make the very sensible observation quoted before the Digression above, incidentally, not so much in the course of a discussion of Kim as in discussion of [Bacon, 1986], which imposes as well as the Boolean closure conditions here under discussion a further condition of 'diagonal resplicing' whose implausibility is also pointed out in [Oddie and Tichý, 1990]. This rather undermines the interest of Bacon's showing that the satisfaction of his conditions collapses the distinction between weak and strong supervenience (and in [Bacon, 1995], Bacon retracts his endorsement of the resplicing condition ${ }^{90}$ ). Kim himself had held that the distinction between strong supervenience and 'reduction' collapses. ${ }^{91}$ That is, the strongly supervenient properties can indeed be reduced to those in the supervenience base, where this is a matter of some strict equivalence holding between each predication ascribing one of the supervenient properties to an individual and the predication ascribing some property in the base to that individual. (For this we need Kim's Boolean closure assumption for the supervenient properties and

89 Since infinitary conjunction and disjunction are assumed here, Kim's restriction to $N$ 's being finite is omitted, as is his restriction to each instantiated property, since we may consider the empty property is in the Boolean closure of any set of properties.

90 Another reaction, to be found in [Rotolo, 2017], has been the suggestion that the underlying modal logic should be weakened to avoid the untoward conclusion. Below, in th Update on Dethroning S5, we look an alternative "weaken the logic" reaction in this general area.

91 A helpful discussion of the relation between supervenience theses and reducibility claims is provided by Stalnaker [2003]. 
not just those in the base, so we can take infinite disjunctions of the latter.) As remarked at the end of Section 1 above, this line of thought is a slight variation, complicated by the fact that we are now considering open rather than closed formulas, on that involved in obtaining from a supervenience claim the disjunction appearing as the last inset formula in Section 1 apropos of [Goranko and Kuusisto, 2018]. There have been several reactions to this finding, one of which is that we need to distinguish various possible interpretations of the necessity operator $\square$ in these strict equivalences, and only on an analytic or 'conceptual necessity' reading - or perhaps as expressing a priori knowability - would they genuinely problematic for the anti-reductionist. As a response like this is presented in [Dreier, 1992, 2015a], the suggestion is that the $\square$ in the potentially worrying strict equivalences is for metaphysical necessity, while the outer $\square$ in formulations of (weak or strong) supervenience (WS) and (SS) above - is for conceptual necessity. (This response is suitable in the case of the supervenience of the moral on the natural, though not in the case of the supervenience of the mental on the physical, for which again a differentiation of readings of " $\square$ " might be suggested: Chalmers Chalmers [1996], Chapter 2, esp. note 16, plays with somewhat similar combinations of metaphysical and nomic necessity along something in this connection. The disanalogy between the moral/non-moral and the mental/physical supervenience theses has often been noted for example at p. 59 of [Blackburn, 1985]. The status of this thesis as a matter of conceptual necessity even in the former case is occasionally contested: for example in [Harrison, 2013, 2017]. Rotolo [2017, p. 19] suggests versions of (WS) and (SS) in which the alethic operators never mind whether they are interpreted metaphysically or conceptually - get replaced by deontic operators, though the motivation for this suggestion, apparently sketched on p. 16 of [Rotolo, 2017], was not clear to this reader.)

Remark 8.6. Note 18 of [Zangwill, 1998] gives a list of several writers Dreier and three others - who have taken a similar line (originally raised in [Blackburn, 1985]). For reasons not gone into here, Zangwill himself does not find this response satisfactory; similarly targeted criticism can also be found at p. 27 of [McLaughlin, 1995]. Klagge [1984, p. 374] last sentence) also objected to this move, offering by way of justification the remark that it "does not seem, for example, that a divine-command theory, according to which moral properties supervene on God's commands 
rather than upon natural properties, involves a logical contradiction or a misuse of moral language." This misunderstands the very broad notion of the natural(istic) in the present context. As Hare [1952, p. 82] put it, "Talking about the supernatural is no prophylactic against 'naturalism'." And it should also be noted that the response in question is offered particularly in reaction to Blackburn [1971, 1985], in the first of which the combination of even - especially, indeed - weak supervenience with a failure of the subjacent properties ${ }^{92}$ to entail the supervenient properties is held to be bad news for an advocate of realism about the latter, essentially because such a position allows no explanation for the absence of the 'mixed worlds' discussed earlier; an argument like that Blackburn presents (though credited by him to Casimir Lewy) - supervenience as a problem for moral realism - is extracted by Horgan and Timmons from the writings of J.L. Mackie and developed by them in [Horgan and Timmons, 1992]. (As well as these references, see [Lewis, 1985, p. 165], for a point observed by Lewis - and note incidentally that we are changing Lewises here, from David to Harry - to have been made long before by G. E. Moore against what turns out to be one line of thought surfacing in Blackburn's discussion.) This non-entailment claim is what is called Hume's Law in the moral case. The formulation from [Stalnaker, 2003, p. 92] "According to Moore, natural properties entail evaluative properties," seems inappropriate, given the way Moore himself had introduced the terminology of entailment into philosophy in the first place - as standing for the converse of deducibility - which rather unfits it for use here, given Stalnaker's continuation of this remark: "but the necessary connection between natural and evaluative is synthetic and

92 R. M. Hare, who placed great emphasis on supervenience in ethics - even if he sometimes went astray in theorising about it (see note 70) - regarded the correct term converse to supervenient as being subjacent - the term used in Remark 8.6 aboverather than the crude though often encountered "subvenient". (Note 3 of [Bacon, 1995] mentions Hare's distaste for "subvenient", though not his preference for "subjacent"; Bacon himself uses instead the phrase "base properties". This was also touched on in note 1 of [Humberstone, 1992], in which the 'subvenient' terminology was nevertheless used.) Etymologically, one can see the point here, with a quasi-temporal priority of the subjacent reflected in the static connotations of "lying beneath" as opposed to the less appropriately dynamic idea (suggested by subvenient) of "coming to be beneath". This contrast manages to capture the (more appropriately) dynamic aspect of G. E. Moore's term for supervenient, namely "consequential", while shedding the latter's potentially misleading causal suggestions, as well, perhaps, as its actually misleading 'resultance' connotations (mentioned in Remark 1.1). 
substantive"; evidently, a thorough consideration of these issues would require coming clean on the individuation of properties. The line of response under consideration here would construe the strict implications which correspond to those displayed in the second last inset formula in Section 1 above - involved from a complete non-moral description of an action or person to the (let's say strongly supervenient) moral description not to be in violation of Hume's Law because the real test of entailment would be the conceptual necessity or apriority of the material implications, rather than their metaphysical necessity. This point is stressed in [McFetridge, 1985, p. 251].

As a slight variant one might consider a theory (whose background logic is a suitable quantified monomodal logic, the $\square$ being intended as metaphysical necessity) embodying the a priori truths or conceptual necessities relating the two classes of properties. A disjunction of (metaphysically) strict equivalences would be provable in the theory, but none of its disjuncts would be, so the theory would not itself be objectionably reductionist.

\section{Update: Dethroning S5?}

The idea of retaining strong supervenience while resisting base-tosupervenient entailments has been resisted by the device of distinguishing metaphysical from conceptual necessity - explicating entailment in terms of the latter, and working with a suitably variegated version of (SS) - but also, more recently, in a quite different way. This novel response depends not on disambiguating the $\square$ in (SS), but instead on attending to the strength of the underlying modal logic presumed to govern this (univocal) operator. The present discussion was foreshadowed at the end of the Digression on Klagge above. Ralph Wedgwood, in particular ([Wedgwood, 2000] and [Wedgwood, 2007, esp. §§9.5, 9.6]), has suggested that we should question the widespread assumption that the logic of metaphysical necessity - the relevant reading of $\square$ for this response - is S5. For current expository purposes there is a complication in that Wedgwood's discussion concerns a form of global supervenience, which if we think of it in the mental-supervenient-on-the-physical form amounts to the claim that where $P^{*}$ is any complete physical description of the world and $Q$ a candidate psychological statement-Wedgwood [2000, p. 410] writes "something is in pain" rather than $Q$ here - then the 
supervenience claim in question seems to commit us to the disjunction of $\left(\mathrm{N}^{+}\right)$and $\left(\mathrm{N}^{-}\right)$here:

$$
\left(\mathrm{N}^{+}\right) \quad \square\left(P^{*} \rightarrow Q\right) \quad\left(\mathrm{N}^{-}\right) \quad \square\left(P^{*} \rightarrow \neg Q\right) .
$$

But in general one or other of these strict implications would seem to represent an inexplicable brute necessity (somewhat recalling Blackburn's discussion, though here $Q$ would be taken as a moral statement and $P^{*}$ a complete description of how things might 'naturally' - i.e., nonmorally - be). But if we set this out explicitly as a modal inference terminating in this disjunction and having premisses in the style of (SS) except without the quantifiers since we are considering this global version, we find ourselves contemplating the inference embodied in the following consequence claim, where $\vdash_{S}$ means that the conjunction of the formulas on the left provably implies in the modal logic $S$ the formula on the right:

$$
\begin{gathered}
\square\left(\left(P^{*} \wedge Q\right) \rightarrow \square\left(P^{*} \rightarrow Q\right)\right), \quad \square\left(\left(P^{*} \wedge \neg Q\right) \rightarrow \square\left(P^{*} \rightarrow \neg Q\right)\right) \vdash_{S} \\
\square\left(P^{*} \rightarrow Q\right) \vee \square\left(P^{*} \rightarrow \neg Q\right) .
\end{gathered}
$$

Wedgwood's point here can be put as the observation that while this would be correct for $S=\mathrm{S} 5$, it would not be correct for $S=$ KT or even $S=$ S4. (Think of these verdicts as pertaining to the general form of the above $\vdash$-statement, with distinct schematic letters, or alternatively distinct sentence letters, in place of $P^{*}, Q$; similarly below.) Since Wedgwood, while dubious about 4 and B (though committed to $T$ ), is mainly interested in criticizing 5 (or $\mathrm{E}$ as he calls it), i.e., $\diamond \varphi \rightarrow \square \diamond \varphi$, the present example is not optimal: the above $\vdash$-claim would be correct for $S=$ S4.3; so (while we return to an issue concerning S4 at the end of this section) the point he has in mind is more precisely illustrated by considering instead the following:

$$
\square\left(\left(P^{*} \wedge Q\right) \rightarrow \square\left(P^{*} \rightarrow Q\right)\right), \quad \diamond\left(P^{*} \wedge Q\right) \vdash_{S} \square\left(P^{*} \rightarrow Q\right),
$$

which is essentially the longer $\vdash$-condition above but with the second formula on the left - i.e., $\square\left(\left(P^{*} \wedge \neg Q\right) \rightarrow \square\left(P^{*} \rightarrow \neg Q\right)\right)$ - deleted, for the following reason.

Proposition 8.7. Among normal modal logics $S \supseteq \mathrm{KT}$, we have

$$
\square((p \wedge q) \rightarrow \square(p \rightarrow q)), \quad \diamond(p \wedge q) \vdash_{S} \square(p \rightarrow q)
$$

if and only if $\vdash_{S} 5$. 
Proof. 'If'. Here we do not need the condition that $S \supseteq \mathrm{KT}$, since evidently

$$
\square((p \wedge q) \rightarrow \square(p \rightarrow q)), \diamond(p \wedge q) \vdash_{\mathrm{K}} \diamond \square(p \rightarrow q),
$$

and 5 , in the form dual to that cited above, would allow us to simplify the right-hand side's " $\triangleright \square$ " to " $\square$ ".

'Only if': Here we use the fact that since

$$
\diamond \square q \vdash_{\mathrm{K}} \diamond(\square q \wedge q) \vee \diamond(\square q \wedge \neg q),
$$

and the negation of the right-hand disjunct of this formula is $\mathrm{KT}$-provable, we have

$$
\diamond \square q \vdash_{\mathrm{KT}} \diamond(\square q \wedge q) .
$$

To complete the proof we show that for $S$ as in the statement of the present Proposition, we also have $\diamond(\square q \wedge q) \vdash_{S} \square q$, since the transitivity of the relation $\vdash_{S}$ (restricted to the case in which there is a single formula on the left, so that we have binary relation between formulas) that $\diamond \square q \vdash_{S} \square q$; that is, $\vdash_{S}$ 5. To show that $\diamond(\square q \wedge q) \vdash_{S} \square q$ for $S$ as in the Proposition, we begin by re-lettering that condition to avoid confusion with the recent use of ( $p$ and) $q$ :

$$
\square((r \wedge s) \rightarrow \square(r \rightarrow s)), \quad \diamond(r \wedge s) \vdash_{S} \square(r \rightarrow s) .
$$

Uniformly substituting $q \rightarrow p$ for $r$ and $q$ for $s$, we get:

$$
\begin{aligned}
& \square(((q \rightarrow p) \wedge q) \rightarrow \square((q \rightarrow p) \rightarrow q)), \\
& \diamond((q \rightarrow p) \wedge q) \vdash_{S} \diamond \square((q \rightarrow p) \rightarrow q) .
\end{aligned}
$$

Now replacing $(q \rightarrow p) \wedge q$ by its equivalent $p \wedge q$, and $(q \rightarrow p) \rightarrow q$ by its equivalent $q$, we get:

$$
\square((p \wedge q) \rightarrow \square q), \diamond(p \wedge q) \vdash_{S} \square q .
$$

Finally, substitute $\square q$ for $p$, rendering the first formula on the left Kprovable, establishing that $\diamond(\square q \wedge q) \vdash_{S} \square q$, as desired.

Two aspects of the 'Only if' part of the above proof merit further clarification. (i): The reference to the KT-provability of the negation of the second disjunct of the right-hand formula in the inset $\vdash$-statement is to the formula $\square(\square q \rightarrow q)$ modally defining the class of range-reflexive frames (frames in which any point accessible to anything is accessible to itself) while the formula employed "to compete the proof", $\diamond(\square q \wedge$ 
q) $\rightarrow \square q$ ), defines the class of frames satisfying the following 'alioEuclidean' condition: for all $w, x, y$, if $w R x, w R y$, and $x \neq y$, then $x R y$. The frames satisfying both conditions are precisely the Euclidean (5-validating) frames. See [Humberstone, 2016, p. 106f.] for this perspective on what is going on in the proof. (ii): The choice of substitution for the variables $r, s$ at the end of the proof may seem to come somewhat out of the blue. Here what is happening this. The condition Proposition 8.7 claims to be equivalent to S's proving the formula 5 is that a specific formula of the form ${ }^{93}$

$$
\square(\varphi \rightarrow \square \psi) \rightarrow(\diamond \varphi \rightarrow \square \psi)
$$

should be S-provable: namely the case in which $\varphi$ is $p \wedge q$ and $\psi$ is $p \rightarrow q$. Notice in this case that the inference from this $\varphi$ to this $\psi$ is not only valid (in classical logic) but archetypally so, in the sense that whenever a formula $\varphi^{\prime}$ has a formula $\psi^{\prime}$ as a classical consequence, the transition from $\varphi^{\prime}$ to $\psi^{\prime}$ can be subsumed under the transition from $\varphi$ to $\psi$ : there is some substitution $s$ with $\varphi^{\prime}$ and $\psi^{\prime}$ classically equivalent to $s(\varphi)$ and $s(\psi)$. (More on this may be found in [Humberstone, 2004b] and [Połacik and Humberstone, 2018].) In particular, we want to choose $s$ in such a way as to subsume the transition from $p \wedge q$ to $q$ (which represents, as it happens, another archetypal inference form) under the $\varphi$ to $\psi$ transition. The general way to subsume the passage from $\varphi^{\prime}$ to $\psi^{\prime}$ as a substitution instance of the passage from $p \wedge q$ to $q,{ }^{94}$ or rather, in our re-lettering, from $r \wedge s$ to $r \rightarrow s$ is substitute $\psi^{\prime} \rightarrow \varphi^{\prime}$ for $r$ and $\psi^{\prime}$ for $s$. For the present choice of $\varphi^{\prime}$ and $\psi^{\prime}$ as $p \wedge q$ and $q$ respectively, so writing $\varphi^{\prime}$ as equivalent to $s(r \wedge s)$ is writing $\psi^{\prime}$ as $s(r \rightarrow s)$ amounts to writing, respectively, $(q \rightarrow(p \wedge q)) \wedge q$ and $(q \rightarrow(p \wedge q)) \rightarrow q$. In the proof above, because of the particular $\varphi^{\prime}, \psi^{\prime}$ involved, we can simplify and write $\psi^{\prime} \rightarrow \varphi^{\prime}$ as $q \rightarrow p$ rather than $q \rightarrow(p \wedge q)$.

Thus the role the provability of the formula mentioned in the statement of Proposition 8.7 could be played instead by speaking of the $S$ provability of all instances of the schema inset in the last paragraph satisfying the further condition that $\varphi \rightarrow \psi$ is provable, or alternatively as having $S$ closed under the rule taking us from $\varphi \rightarrow \psi$ to $\square(\varphi \rightarrow \square \psi) \rightarrow(\diamond \varphi \rightarrow \square \psi)$. Conspicuously among the formulas thereby

93 The form in question is one half of the biconditional schema inset before $\left(\mathrm{SSS}^{\prime \prime \prime}\right)$ at the end of the Digression on Klagge above.

94 This is recorded in the second row of the table labelled 'Figure 4' on p. 53 of [Humberstone, 2004b]. 
delivered are the instances of this schema in which $\varphi$ and $\psi$ are the same formula, so without loss of generality we are in effect here dealing with the modal formula:

$$
\square(p \rightarrow \square p) \rightarrow(\diamond p \rightarrow \square p)
$$

This formula modally defines the class of frames satisfying a variant of van Benthem's second order condition of 'Safe Return' [van Benthem, 2001, p. 347], which could in the same vein be dubbed 'Safe Journey' (as a condition on frames $\langle W, R\rangle$, quantifiers ranging over $W$ ): If $w R x$ and $w R y$ then there are points $u_{1}, \ldots, u_{n}$ with $x=u_{1} y=u_{n}$ and $u_{i} R u_{i+1}$ for $i<n$ and $w R u_{i}$ for each $i$. (The special case of $n=2$ is the Euclidean condition.) The antecedent of this formula can be regarded as recording a weak kind of noncontingency for $p$, distinct in S4 for example (though not in S5) from the more familiar kind of noncontingency recorded in its consequent: see note 85 .

Remark 8.8. The modal formula with which van Benthem is concerned in the passage just cited has the final $\square p$ replaced by $p$. The proof he gives that this formula modally defines the class of frames satisfying the Safe Return condition makes an appeal to reflexivity at one point but this is not required: to avoid it, define $V(p)$ to comprise what there appears as $y$ together with all successors of good points - with good as defined there. To show the unobvious direction of the claim that the modal formula as above, with final consequent $\square p$ rather than $p$, modally defines the class of frames satisfying the Safe Journey condition, a minor modification of this argument is called for, along the following lines. Suppose we have a frame not satisfying the condition. Thus there are elements $w, x, y$ with $w R x$ and $w R y$ and no $R$-chain of $R$-successors of $w$ taking us from $x$ to $y$. Let a good' point be any point $z$ such that there is an $R$-chain of $w$-successors starting with $x$ and terminating in $z$. Letting $V(p)$ be the union of $\{x\}$ with the set of successors of good' points gives a (partial description of a) model in which at $w \square(p \rightarrow \square p)$ and $\diamond p$ are true while $\square p$ is not. As van Benthem has observed (p.c.), this choice of $V(p)$ is one that would be obtained by following the 'minimization' strategy described in [van Benthem, 2005]. (Incidentally, in Example 9 on p. 709 of that paper, where the present example - the version with consequent $p$ rather than $\square p$-is treated, the frame condition is given erroneously as "every point $x$ with an $R$-successor $y$ can be reached from $y$ by a finite sequence of successive $R$-steps"; these words should be followed by "each point in which is itself an $R$-successor of $x "$.) 
Formulas of the form $\square(\varphi \rightarrow \square \varphi)$, or more generally $\square(\varphi \rightarrow \square \psi)$, have a special place in Wedgwood's discussion, since - to put it in terms of the latter formulation - they make room for the idea that the truth of $\square \psi$ may be explained by the truth of $\varphi$, even if $\varphi$ itself is only contingently true. Let us return to the earlier example with $\psi$ and $\psi$ as respectively $P^{*} \wedge Q$ and $P^{*} \rightarrow Q$, with $P^{*}$ a fully specific description of a world in subjacent terms and $Q$ a description in supervenient terms (relative to whatever supervenience claim is under discussion):

$$
\square\left(\left(P^{*} \wedge Q\right) \rightarrow \square\left(P^{*} \rightarrow Q\right)\right), \quad \diamond\left(P^{*} \wedge Q\right) \vdash_{S} \square\left(P^{*} \rightarrow Q\right) .
$$

This was something a good choice of $S$ was meant to block since it seemed to land us with the brute necessity $\square\left(P^{*} \rightarrow Q\right)$. (Recall we are dealing in metaphysical necessity here, so the earlier worry that this would be a violation of Hume's Law or the analogous barrier principle in whatever supervenience arena was at issue.) The most we had (for $S=$ KT or S4, though Wedgwood is also suspicious about 4 on grounds we do not go into here) was the weaker conclusion $\diamond \square\left(P^{*} \rightarrow Q\right)$. One might react: yes, but what if $P^{*} \wedge Q$ is not just possible but true? Then even the favoured 5-eschewing candidates for $S$ will allow us the corresponding $\vdash$-claim, since we have no $\diamond$ to contend with in the second premiss and can drop the $\square$ from the first (as $S \supseteq \mathrm{KT}$ ), with Modus Ponens doing the rest:

$$
\square\left(\left(P^{*} \wedge Q\right) \rightarrow \square\left(P^{*} \rightarrow Q\right)\right), \quad P^{*} \wedge Q \vdash_{S} \square\left(P^{*} \rightarrow Q\right) .
$$

I take it that in Wedgwood's view that this would then be precisely a case in which a contingent $\varphi$ (i.e., a case in which $\varphi \wedge \neg \square \varphi, \varphi$ being $P^{*} \wedge Q$ ) explains the necessity of something (here $P^{*} \rightarrow Q$ ). The analogous situation with strong supervenience has, corresponding to this last $\vdash$-statement, the following with $P *$ and $Q$ taken as open formulas, here written as though atomic monadic predicates:

$$
\begin{gathered}
\square\left(\exists x\left(P^{*} x \wedge Q x\right) \rightarrow \square \forall x\left(P^{*} x \rightarrow Q x\right)\right), \\
\exists x\left(P^{*} \wedge Q x\right) \vdash_{S} \square \forall x\left(P^{*} x \rightarrow Q x\right),
\end{gathered}
$$

and again the cost of putting a $\diamond$ on the second premiss is the addition of an ineliminable $\diamond$ on the conclusion, and this blocks the strict implications required for Kim-style "no supervenience without reducibility" claims.

The leftmost formula in the last inset $\vdash$-statement, prefixed by " $\exists P^{*}$ " is a variation on the assertion that $Q$ is strongly supervenient on the class $\mathbb{P}$ of properties, with the notation understanding that $P^{*}$ can be taken 
as a maximal (consistent) property in $\mathbb{P}$. It is worth comparing this (SS) above, for which purpose we repeat the latter here but for the supervenience of applied to a single property, and re-letter the Wedgwood-style supervenience formulation just arrived at (since the $P$ and $Q$ are the other way round, here we interchange them except when they flank $\wedge$ ), dropping the asterisks and putting in an existential quantifier at the same time:

$$
\begin{aligned}
& \square \forall x(P x \rightarrow \exists Q \in \mathbb{Q}(Q x \wedge \square \forall y(Q y \rightarrow P y))) \\
& \square \exists Q \in \mathbb{Q}(\exists x(P x \wedge Q x) \rightarrow \square \forall y(Q y \rightarrow P x))
\end{aligned}
$$

So that one has the first quantifier universal in both cases, we could reformulate the 'new style' version of strong supervenience of $P$ on $\mathbb{Q}$ :

$$
\square \exists Q \in \mathbb{Q} \forall x((P x \wedge Q x) \rightarrow \square \forall y(Q y \rightarrow P x)) .
$$

The most conspicuous difference apparent in the new version is that while the promised $Q \in \mathbb{Q}$ is not represented as depending on the $x$ with property $P$. Exactly what additional assumptions or qualifications would be needed to render this new form equivalent to (SS) would be worth investigating, though this will not be done here. (Evidently there should be a restriction on the " $\exists Q \in \mathbb{Q}$ " if $\mathbb{Q}$ contains unpossessable properties - as it would if $\mathbb{Q}$ is closed under Boolean combinations - or indeed properties incompatible with $P$, since then the conditional in its scope will be vacuously true.)

Let us return to Wedgwood's own main idea of weakening the modal logic in use from S5 so as to block Kim-style arguments from strong supervenience to reduction, and close, more specifically, with an outline of the interesting reaction to this idea voiced in [Schmitt and Schroeder, 2011] (appearing also as Chapter 5 of Schroeder [2014]). ${ }^{95}$ What follows aims to give a brief airing of some of the issues arising in this debate, rather than to take sides.

As a first approximation to the Schmitt-Schroeder reaction, let us recall the fact that generally, though not quite universally, the weaker one's logic is from a deductive point of view, the stronger it is from a

95 The latter version cleans up a typographical mishap from footnote 11 of [Schmitt and Schroeder, 2011], in the last three lines of which all occurrences of negation should be occurrences of $\square$. Like Wedgwood, Schmitt and Scheider discuss also global supervenience and they accordingly refer (e.g., p. 146 of [Schmitt and Schroeder, 2011]) to Kim-Jackson arguments, rather than just (as above) Kim-style arguments. 
discriminatory point of view: fewer equivalences makes for more distinctions. ${ }^{96}$ Thus we need to be alert to the possibility that weakening our modal logic (for metaphysical necessity) from S5 to something such as $\mathrm{S} 4$ or $\mathrm{KT}$, what we witness is not so much that a certain premiss (a supervenience thesis) no longer yields a certain conclusion (reductionism interpreted in terms of necessary coextensiveness), but rather the dissolution what we thought of a single premiss and a single conclusion into a multiplicity alternative versions of the premiss and the conclusion which can be distinguished from each other only now that we have weakened the logic. We are then left with the question of which versions of the old premiss - thought of informally but now with distinct natural formalizations no longer equivalent - have which versions of the old conclusion, similarly conceived, as logical consequences.

But that is only a first approximation Schmitt and Schroeder's opening moves, since they introduce the discussion not in terms of weakening the modal logic S5 to that of S4 or KT, but of weakening the theories of the Kripke frames modally defined by those logics (i.e., the frames validating precisely the theorems of the logics in question) - modulating to object-language modal formulations only later. In particular, for given classes A and B (to use their notation) of properties, concerning which we are interested in the supervenience of the A-properties on the B-properties, [Schmitt and Schroeder, 2011, p. 136] (or [Schroeder, 2014, p. 99]), introduce the following binary relation between worlds, where the notation is intended to recall "strong supervenience condition": $\operatorname{SSC}\left(w_{1}\right.$, $w_{2}$ ) iff for any individuals $x, y$ if for each B-property $x$ has that property at $w_{1}$ just in case $y$ has that property at $w_{2}$, then for each A-property $x$ has that property at $w_{1}$ just in case $y$ has that property at $w_{2} \cdot{ }^{97}$ But which worlds $w_{1}, w_{2}$ are relevant, when we are evaluating a (strong or interworld) supervenience claim at a world $w$ ? Schmitt and Schroeder consider two options which they call relative and absolute, but since the accessibility relation $R$ is involved in both cases, I would prefer to distinguish according to whether the worlds are considered in series or in parallel (for which, recall that $R(w)$ denotes the set of worlds accessible

96 References to the literature on this theme, as well as some exceptions to the generalization in question, can be found in [Humberstone, 2005b].

97 This formulation is tailored to allowing overlapping domains. Schmitt and Schroeder give a characterization presuming the different worlds; domains are disjoint, in note 4 of p. 136 in [Schmitt and Schroeder, 2011] (or note 4 of p. 99 in [Schroeder, 2014]). 
by $R$ to $w)$ :

$$
\begin{array}{cl}
\text { In Series: } & \forall w_{1} \in R(w) \forall w_{2} \in R\left(w_{1}\right) \cdot \operatorname{SSC}\left(w_{1}, w_{2}\right) \\
\text { In Parallel: } & \forall w_{1} \in R(w) \forall w_{2} \in R(w) \cdot \operatorname{SSC}\left(w_{1}, w_{2}\right)
\end{array}
$$

Schmitt and Schroeder point out that the first of these implies the second in the presence of the additional assumption that $R$ is transitive, while the second implies the first in the presence of the additional assumption that $S$ is Euclidean, so they are equivalent over frames for S5, though not over (arbitrary) frames for S4 or KT. (The reflexivity of $R$ is not up for negotiation. ${ }^{98}$ ) They also point out that modal object language principle like (SS) and (SSS), in which there is, as we recalled in the Digression on Klagge above, no modal embedding, on the one hand, and (SSS'"'), in which there is, correspond to the 'in parallel' and 'in series' formulations of strong supervenience, though they doubt ([Schmitt and Schroeder, 2011], §6) that there are any good reasons for following Wedgwood in preferring the 'in series' formulations of such principles, though they agree that this characterization of supervenience does not lead to problematic a reducibility-related conclusion. At the 'conclusion' end of such would-be derivations there also arises an issue of discriminable theses once the logic is weakened. Schmitt and Schroeder [2011, note 11] raise issues in this vicinity when discussion property identity, but here I will put the point in terms of propositional identity and then a unilateralized version of this idea.

Suppose we say that a binary connective, not necessarily primitive, which I will write as $\approx$ in the vocabulary of a modal logic is a propositional identity connective if for every context $C(\cdot)$ the logic - here for familiarity conceived of as a set of provable formulas - contains all formulas of the following forms:

$$
(\approx \mathrm{I}) \quad \varphi \approx \varphi \quad(\approx \mathrm{E}) \quad(\varphi \approx \psi) \rightarrow(C(\varphi) \rightarrow C(\psi)) .
$$

Here the labelling is intended to recall the rules of identity introduction and identity elimination in a natural deduction system for first order logic with identity (the identity predicate, that is, not the current binary connective). It is well known that strict equivalence is a propositional identity connective in a normal modal logic if and only if that logic ex-

98 And this slightly messes up the elegant symmetry, in fact, since of courseand this is something Schmitt and Schroeder mention - we can't have T and 5 without also having 4 (or, in terms of the model theory, reflexive Euclidean relations are all transitive), even though we can have $\mathrm{T}$ and 4 without having 5 . 
tends $\mathrm{S} 4 ;^{99}(\approx \mathrm{E})$ in particular seems like a necessary condition for $p \approx q$ to be amenable to an informal interpretation along the lines of: the proposition that $p$ coincides with the proposition that $q$. Thus a strict equivalence of the supervenient on some compound of the base propositions (or properties, mutatis mutandis) only deserves to be regarded as any kind of reduction of the former to the latter for logics at last as strong as S4, in which this equivalence can qualifies as a propositional (or property) identity. In the discussion of (2.3) and (2.4) in Section 2 this idea came up in the reference to synonymy as understood by Smiley, and a minor model-theoretic adaptation ("synonymy at a point in a model") of this idea.

Turning, now somewhat speculatively, to the envisaged unilateral version of this idea, we define a binary connective $\rightsquigarrow$ to constitute, let us say - taking inspiration from (and intending no offence toward) the practitioners of the 'deep inference' style of proof theory (e.g. [Brünnler, 2009]) - a deep implication connective (in a given logic), if the set of theorems is closed under the analogues of $(\approx \mathrm{I})$ and $(\approx \mathrm{E})$ with " $\approx$ " replaced by " $\rightsquigarrow$ " in both cases and in the latter case a restriction to contexts $C(\cdot)$ with the property that $C(p \wedge q) \rightarrow C(p)$ is provable. ${ }^{100}$ (Note that while the rightmost $\rightarrow$ in the schematic formulation of $(\approx \mathrm{E})$ could equivalently be written as $\leftrightarrow$, that is not so in the case of $(\rightsquigarrow \mathrm{E})$, since embedding under $\neg$ does not satisfy the condition on $((\cdot)$.) By analogy with the case of propositional identity, one might say that a strict implication presents a threat to 'Hume's Law' type worries if it is a deep implication, and

99 See [Cresswell, 1965] or [Wiredu, 1979] indeed in the formulation (1.4) at the end of our opening section we notated the necessitated biconditionals of Goranko and Kuusisto's condition using $\approx$, taking advantage of the fact that their assumed background logic was S5. The above schemata can be found packaged as sequentto-sequent rules on p.603f. of [Humberstone, 2011] where they are used in a unique characterization argument.

100 The condition on $C(\cdot)$ involving the transition from a conjunction to a conjunct can be replaced with any other classically archetypal such transition or by a suitably cleaned up version of the conditional ('monotone') condition that $C(\cdot)$ should satisfy: whenever $\chi_{1} \rightarrow \chi_{2}$ is provable so is $C\left(\chi_{1}\right) \rightarrow C\left(\chi_{2}\right)$. (Taken at face value such a formulation is potentially impredicative in the case in which we want to add as a new connective $\rightsquigarrow$ satisfying these conditions, since $(\rightsquigarrow I)$ itself is employed in part to specify what is provable and can't be governed by a condition presuming that specification already to be available. This is not like a rule of proof telling us that if this - e.g., $\varphi$ - is provable, then so is $\square \varphi$ - because in the case currently envisaged the condition on $(\rightsquigarrow \mathrm{E})$ addresses the provability of all formulas $C\left(\chi_{1}\right) \rightarrow C\left(\chi_{2}\right)$ for which $\chi_{1} \rightarrow \chi_{2}$ is provable.) 
observe that in only those normal modal logics which extend S4 is strict implication a deep implication connective. The idea here is again that if we want to simulate conceptual connections using material implication alongside a notion of metaphysical necessity extension is apt to vary from world to world, we need to enforce some stability by taking into account the arbitrary depth of modal embedding of the implication in question. But since this is not a line of thought explicitly entertained by Wedgwood or his critics, let us leave matters there.

\section{Postscript: A Contrast with Quantificational Dependence Logic?}

Let me reproduce a parenthetical warning bearing on sections 1-3 above from [Humberstone, 1987, p. 102f.], mentioned in note 2 above; the nonparenthetical part of the passage, ${ }^{101}$ which supplies a context for the warning, incidentally anticipates the 'slash' device introduced in [Hintikka and Sandu, 1989] with the superscripted bracketed variables.

We could do this (perhaps) by the device of writing, when in the scope of a quantifier $\forall u$ either the usual existential quantifier or something of the form $\exists v^{[u]}$ to be read: "there is some $v$ not depending on the $u$ in question', so understood that any formula containing $\forall u \exists v^{[u]}(\ldots)$ within it is equivalent to the result of replacing such a contained occurrence by ' $\exists v \forall u(\ldots)$ '. (Warning: this is no kind of restricted quantifier it's not as if some objects were dependent upon or independent of other objects.)

Here is how this warning appears in [Väänänen and Hodges, 2010]:

The sentence

$x_{n}$ is determined by $x_{1}, \ldots, x_{n-1}$

is meaningless of the variables $x_{1}, \ldots, x_{n}$ stand for particular things.

For example, is 5 dependent on 2 and $\omega$ ? This way lies nonsense.

Similar sentiments are to be found in Hodges' papers from the 1990s and I cite this one partly because of the title of the article in which it appears, which precisely articulates Väänänen's distinctive move in passing from branching quantifiers and IF logic to quantificational de-

101 In quoting this passage here, I correct a typo: the first occurrence of " $\forall u$ " appeared confusingly in [Humberstone, 1987] as " $\forall v$ ". 
pendence logic: "Dependence of Variables Construed as an Atomic Formula". What is distinctive here is that D or, as they write it, $=(\cdots)$ is a predicate symbol, whether or not one treats it as a single predicate symbol or, insisting on unigrade predicates, as a family of such symbols. One might have thought that an atomic formula then precisely says that the current values of the variables involved stand in a certain relation, so if the variables range over, say, as in the example of the quoted passage, ordinals, then this will be a relation among ordinals which is exactly what the passage denies. But such a reaction would be premature, since on Hodges' semantics the semantic assignment to a variable is not one of the things over which the variables are usually taken to range, but something at a higher level in the set-theoretic hierarchy. As Hodges summarises matters on p. 120 of [2007]: "It turned out that the main idea needed was to think of formulas as satisfied not by assignments to their variables, but by sets of assignments." The suggestion Väänänen and Hodges [2010, p. 828] makes of how constructions like this are interpreted in natural language - via type-shifting also seems promising; also of interest is the question of whether someone denying on metaphysical grounds that identity is a relation between individuals is thereby debarred from using "=" in atomic formulas: after all, dependence logic features a dependency predicate not interpreted as such a relation. ${ }^{102}$ But let us turn to what appears at first sight to be a contrast between the quantificational and the modal incarnations of dependence logic. (Under the latter label for convenience I subsume the 'pure' or de-modalized Goranko-Kuusisto version of dependence logic $\mathcal{D}$ over which they prefer their own $\mathcal{L}_{\mathrm{D}}$ version of dependence logic.)

In the modal case of course $\mathrm{D}$ is not a predicate symbol but a connective and so dependence statements do not appear as atomic formulas (as in the title of [Väänänen and Hodges, 2010]), though one could construe them as such by treating the formulas in the scope of D-ideally not requiring these to be sentence letters - as names, for any given model as the propositions they express in the model (i.e. the sets of worlds at which they are true in that model, or in the possibilities framework, the regions over which they are true - i.e., the over possibilities and also the empty region). But now what happens to the warning above? Under what-

102 See [Wehmeier, 2004, 2012] for a presentation - complete with the historical precedents for this package - of the two ingredients it is speculated here might come apart: (1) there is no such relation as identity and (so) (2) the identity predicate is to be avoided. 
ever name - dependence, determination or supervenience - we are in this case dealing with genuine relation between propositions, holding between propositions $P$ and $Q$ (relative to some model) when any worlds agreeing on membership in $P$ agree on membership in $Q$. (This formulation is tailored to determination; for supervenience or dependence it is the converse of this relation that is at issue.) This disanalogy with the quantificational case can be pressed further with the observation that in modal dependence logic no quantifiers - and in particular no existential quantifiers are present, so the question of the dependence of a choice of witness on choices for outlying universal quantifiers does not actually arise.

The right response to this claimed disanalogy between the quantificational case and the cases of $\mathcal{D}$ and $\mathcal{L}_{\mathrm{D}}$ seems to be this: don't think of the propositional variables, or more generally the formulas of these languages, as standing for propositions (sets of worlds, relative to any model) but rather as standing for truth-values (relative to any model and world); ${ }^{103}$ see was the suggested informal gloss (on D-formulas in the third sentence of Section 1 above. In terms of the distinction referred to above the propositions are the semantic values rather than what the propositional variables range over. This response is essentially that suggested in the remark of [Väänänen, 2008, p. 238] to the effect that "one cannot meaningfully claim that the propositional symbols true or false in one single node manifest any kind of dependence", at least if is this is construed as meaning that their (local) truth-values cannot be said to manifest dependence or independence. (See also the explanation of uniformity in Remark 5.1.) However, when explaining his dependence formulas earlier on the same page of [Väänänen, 2008], and writing " $=\left(p_{1}, \ldots, p_{n}, q\right)$ " for such a formula "with the intuitive meaning that $q$ depends only on $p_{1}, \ldots, p_{n}$ " and adding that "[t]he quantified $p_{1}, \ldots, p_{n}$ and $q$ can be propositions or individuals," the former being what is at issue in [Väänänen, 2008], things have gone wrong: the appropriate analogue to an individual is not a proposition but a truth-value. And in this analogy what correspond to propositions - which we can think of sufficiently indiscriminately loosely either as sets of worlds or as the characteristic functions of such sets - are not individuals but functions with individuals as values. ${ }^{104}$ In the modal and temporal cases, these

103 Taking "stands for" as "has for its extension" this contrast and its terminological repercussions has been explored in [Lewis, 1974].

104 Characterizing propositions like this is like taking properties in sensu stricto, 
functions have respectively worlds and times as their arguments, while in the quantificational cases they have assignments to individual variables as their arguments.

Acknowledgments. I am grateful to Valentin Goranko, Antti Kuusisto and Jie Fan for keeping me abreast of their thoughts on these issues, and, for assistance in various further ways during the writing, to Johan van Benthem, Sam Butchart, Valentin Goranko, Wes Holliday, Rohan French and Jouko Väänänen, as well as to a referee for Logic and Logical Philosophy for helpful corrections.

\section{References}

Abramsky, S., and J. Väänänen, 2009, "From IF to BI: A tale of dependence and separation", Synthese 167: 285-308. DOI:10.1007/s11229-008-9415-6 Aher, M., 2011, "Free choice in deontic inquisitive semantics (DIS)", pages 22-31 in M. Aloni, V. Kimmelman, F. Roelofsen, G. W. Sassoon, K. Schulz, and M. Westera (eds.), Logic, Language and Meaning (18th Amsterdam Colloquium), Lecture Notes in Computer Science \#7218, Springer, Berlin, Heidelberg. DOI: 10.1007/978-3-642-31482-7_3

Alonso-Ovalle, L., 2005, "Distributing the disjuncts over the modal space", pages 75-86 in L. Bateman and C. Ussery (eds.), Proceedings of the North East Linguistics Society, vol. 35, Amherst, Mass., Graduate Linguistic Student Association (U. Mass.) Publications. DOI: 10.1.1.472.6497

Bacon, J., "Supervenience, necessary coextension and reducibility", Philosophical Studies 49: 163-176. (Reprinted as Chapter 13 in Kim [2002].) DOI: 10. 1007/BF00354332

Bacon, J., 1995, "Weak supervenience supervenes", pages 101-109 in [Savellos and Yalçin, 1995]. DOI: 10.1017/CB09780511663857.005

Bader, R. M., 2012, "Supervenience and infinitary property-forming operations", Philosophical Studies 160: 415-423. DOI: 10.1007/s11098-0119727-0

Bartsch, R., 1986, "The construction of properties under perspectives", Journal of Semantics 5: 293-320. DOI: 10.1093/jos/5.4.293

Bennett, K., 2004, "Global supervenience and dependence", Philosophy and Phenomenological Research 68: 501-529. DOI: 10.1111/j.1933-1592. 2004.tb00364.x

as explained in Remark 8.1. If one wants to speak of moral propositions and the like, as Blackburn does (especially in [1971]), then a notion of a 'pre-proposition' would be called for. 
Bigelow, J., 1988, The Reality of Numbers: A Physicalist's Philosophy of Mathematics, Oxford University Press, Oxford.

Blackburn, S., 1971, "Moral realism", pages 101-124 in J. Casey (ed.), Morality and Moral Reasoning, Methuen, London.

Blackburn, S., 1984, Spreading the Word, Oxford University Press, Oxford.

Blackburn, S., 1985, "Supervenience revisited", pages 47-67 in I. Hacking (ed.), Exercises in Analysis: Essays by Students of Casimir Lewy, Cambridge University Press, Cambridge. (Reprinted as Chapter 2 in Kim [2002].)

Bonevac, D., 1991, "Semantics and supervenience", Synthese 87: 331-361. DOI: $10.1007 / \mathrm{BF} 00499816$

Brueckner, A., 2002, "Blackburn's modal argument against moral realism", Theoria 68: 67-70. DOI: 10.1111/j.1755-2567.2002.tb00120.x

Brünnler, K., 2009, 'Deep sequent systems for modal logic', Archive for Mathematical Logic 48: 551-577. DOI: 10.1007/s00153-009-0137-3

Burgess, J.P., 2003, "A remark on Henkin sentences and their contraries", Notre Dame Journal of Formal Logic 44: 185-188. DOI: 10.1305/ndjfl/ 1091030856

Cariani, F., 2013, "Ought" and resolution semantics", Nô̂s 47: 534-558. DOI: $10.1111 / j .1468-0068.2011 .00839 . x$

Chalmers, D. J., 1996, The Conscious Mind, Oxford University Press, Oxford.

Chalmers, D. J., 2004, "Epistemic two-dimensional semantics", Philosophical Studies 118: 153-226. DOI: 10.1023/B:PHIL.0000019546.17135.e0

Ciardelli, I., 2009, "Inquisitive semantics and intermediate logics", MSc Thesis, University of Amsterdam; Institute for Logic, Language and Computation, Amsterdam.

Ciardelli, I., 2016a, Questions in Logic, Institute for Logic, Language and Computation (ILLC Dissertation Series), Amsterdam.

Ciardelli, I., 2016b, "Dependency as question entailment", pages 129-181 in S. Abramsky et al. (eds.), Dependence Logic, Springer, Cham (Switzerland). DOI: $10.1007 / 978-3-319-31803-5 \_8$

Ciardelli I., J. Groenendijk and F. Roelofsen, 2014, "Information, issues, and attention", pages 128-166 in D. Gutzmann, J. Köpping and C. Meier (eds.), Approaches to Meaning: Composition, Values, and Interpretation, Brill, Leiden. DOI: 10.1163/9789004279377_007

Ciardelli, I., J. Groenendijk and F. Roelofsen, 2015a, "On the semantics and logic of declaratives and interrogatives", Synthese 192: 1689-1728. DOI: 10. 1007/s11229-013-0352-7

Ciardelli, I., J. Groenendijk, and F. Roelofsen, 2015b, "Inquisitive semantics", ESSLLI Lecture notes.

Ciardelli, I., and F. Roelofsen, 2011, "Inquisitive logic", Journal of Philosophical Logic 40: 55-94. DOI: $10.1007 / \mathrm{s} 10992-010-9142-6$ 
Correia, F., 2010, "Grounding and truth-functions", Logique et Analyse 53: 251-279.

Cresswell, M. J., 1965, "Another basis for S4", Logique et Analyse 8: 191-195.

Cresswell, M. J., 1988, "Necessity and contingency", Studia Logica 47: 145-149. DOI: $10.1007 / \mathrm{BF} 00370288$

Dancy, J., 1981 "On moral properties", Mind 90: 367-385. DOI: 10.1093/ mind/XC.359.367

Dancy, J., 2004, Ethics Without Principles, Oxford University Press, Oxford. DOI: $10.1093 / 0199270023.001 .0001$

Davies, M., 1992, "Perceptual content and local supervenience", Proceedings of the Aristotelian Society 92: 21-45. DOI: 10.1093/aristotelian/92.1.21

Davies, M., and L. Humberstone, 1980, "Two notions of necessity", Philosophical Studies 38: 1-30. DOI: 10.1007/BF00354523

DePaul, M.R., 1987, "Supervenience and moral dependence", Philosophical Studies 51: 425-439. DOI: 10.1007/BF00354046

Divers, J., 1996, "Supervenience for operators", Synthese 106: 103-112. DOI: 10.1007/BF00413616

Došen, K., 1989, "Sequent systems and groupoid models: II", Studia Logica 48: 41-65. DOI: 10.1007/BF00370633

Dowty, D. R., 1977, "Toward a semantic analysis of verb aspect and the English 'imperfective' progressive", Linguistics and Philosophy 1: 45-77. DOI: 10. 1002/9780470758335. ch11

Dreier, J.A., 1990, "Internalism and speaker relativism', Ethics 101: 6-26. DOI: $10.1086 / 293257$

Dreier, J.A., 1992, "The supervenience argument against modal realism", Southern Journal of Philosophy 30: 13-38. DOI: 10.1111/j.2041-6962. 1992.tb00636.x

Dreier, J.A., 2015a, 'Explaining the quasi-real", Chapter 11 in R. ShaferLandau (ed.), Oxford Studies in Metaethics, Vol. 10, Oxford University Press, Oxford. DOI: 10.1093/acprof : oso/9780198738695.001.0001

Dreier, J.A., 2015b, "Another world: the metaethics and metametaethics of reasons fundamentalism", pages 155-171 in R. Johnson and M. Smith (eds.), Passions and Projections: Themes from the Philosophy of Simon Blackburn, Oxford University Press, Oxford. DOI: 10.1093/acprof : oso/ 9780198723172.003 .0009

Dummett, M., 1981, Frege: Philosophy of Language (Second Edn.) Duckworth, London.

Ebbing, J., L. Hella, A. Meier, J.-S. Müller, J. Virtema and H. Vollmer, 2013, "Extended modal dependence $\operatorname{logic} \mathcal{E} \mathcal{M D} \mathcal{L}$ ", pages $126-137$ in L. Libkin, U. Kohlenbach and R. de Queiroz (eds.), Logic, Language, Information, and Computation (20th International Workshop, WoLLIC 2013, Darmstadt, 
Germany), LNCS 8071, Springer-Verlag, Berlin. DOI: 10.1007/978-3-64239992-3_13

Elliot, R., 1987, "Moral realism and the modal argument", Analysis 47: 133137. DOI: $10.1093 /$ analys/47.3.133

Fagin, R., 1977, "Functional dependencies in a relational database and propositional logic", IBM Journal of Research and Development 21: 534-544. DOI: $10.1147 / \mathrm{rd} .216 .0534$

Fan, J., 2016, "A modal logic of supervenience", to appear in Notre Dame Journal of Formal Logic. arXiv:1611.04740v1

Fine, K., 2014, "Truth-maker semantics for intuitionistic logic", Journal of Philosophical Logic 43: 549-577. DOI: 10.1007/s10992-013-9281-7

Font, J. M., R. Jansana and D. Pigozzi, 2006, "A survey of abstract algebraic logic", Studia Logica 74: 13-97. DOI: 10.1023/A:1024621922509

Galliani, P., 2013a, "The dynamification of modal dependence logic", Journal of Logic, Language and Information 22: 269-295. DOI: 10.1007/s10849013-9175-7

Galliani, P., 2013b, "Epistemic operators in dependence logic", Studia Logica 101: 367-397. DOI: $10.1007 / \mathrm{s} 11225-013-9478-3$

Galliani, P., and J. Väänänen, 2014, "On dependence logic", pages 101-110 in A. Baltag and S. Smets (eds.), Johan van Benthem on Logic and Information Dynamics, Springer, Cham (Switzerland). DOI: 10.1007/978-3-31906025-5_4

Geurts, B., 2005, "Entertaining alternatives: disjunctions as modals", Natural Language Semantics 13: 383-410. DOI: 10.1007/s11050-005-2052-4

Gibbard, A., 1992, Wise Choices, Apt Feelings: A Theory of Normative Judgment, Oxford University Press, Oxford.

Gibbard, A., 2003, Thinking How to Live, Harvard University Press, Cambridge, MA.

Glanzberg, M., 2001, "Supervenience and infinitary logic", Nô̂s 35: 419-439. DOI: $10.1111 / 0029-4624.00304$

Goldstick, D., and B. O'Neil, 1988 "'Truer'”, Philosophy of Science 55: 583597. DOI: $10.1086 / 289462$

Goodman, N., 1977, The Structure of Appearance (Third edn.), D. Reidel, Dordrecht. (First Edn. 1951.) DOI: 10.1007/978-94-010-1184-6

Goranko, V., and A. Kuusisto, 2018, "Logics for propositional determinacy and independence", Review of Symbolic Logic (published online). DOI: 10.1017/ S1755020317000272

Grimes, T. R., 1988, "The myth of supervenience", Pacific Philosophical Quarterly 69: 152-160. DOI: 10.1111/j.1468-0114.1988.tb00305.x 
Groenendijk, J., 2009, "Inquisitive semantics: Two possibilities for disjunction", pages 80-94 in P. Bosch, D. Gabelaia and J. Lang (eds.), Logic, Language, and Computation: 7th International Tbilisi Symposium on Logic, Language, and Computation, ('TbiLLC 2007', Tbilisi, Georgia, October 1-5, 2007), LNAI \#5422, Springer, Berlin. DOI: 10.1007/978-3-642-00665-4_8

Groenendijk, J., and F. Roelofsen, 2010, "Radical inquisitive semantics", Presented at the Sixth International Symposium on Logic, Cognition, and Communication at the University of Latvia. Online version

Hare, R. M., 1952, The Language of Morals, Oxford University Press, Oxford.

Hare, R. M., 1984, "Supervenience", Proceedings of the Aristotelian Society, Supplementary 58: 1-16. DOI: 10.1093/aristoteliansupp/58.1.1

Harrison, G., 2013, "The moral supervenience thesis is not a conceptual truth", Analysis 73: 62-68. DOI: 10.1093/analys/ans140

Harrison, G., 2017, "The Dubious moral supervenience thesis", pages 89-104 in B. Brożek, A. Rotolo and J. Stelmach (eds.), Supervenience and Normativity, Law and Philosophy, Springer, Dordrecht. DOI: 10.1007/978-3-31961046-7_5

Heil, J., 1995, "Supervenience Redux", pages 158-169 in [Savellos and Yalçin, 1995]. DOI: $10.1017 /$ CB09780511663857.009

Hella, L., K. Luosto, K. Sano and J. Virtema, 2014, "The expressive power of modal dependence logic", pages 294-312 in R. Goré, B. Kooi and A. Kurucz (eds.), Advances in Modal Logic, vol. 10, College Publications, London.

Hellman, G., 1985, "Determination and logical truth", Journal of Philosophy 82: 607-616. DOI: $10.2307 / 2026415$

Hellman, G., 1992, "Supervenience/determination a two-way street? Yes, but one of the ways is the wrong way!", Journal of Philosophy 89: 42-47. DOI: $10.2307 / 2026892$

Hellman, G., and F. Wilson, 1975, "Ontology, determination, and reduction", Journal of Philosophy 72: 551-564. DOI: 10.2307/2025067

Hintikka, J., 1972, "Leibniz on plenitude, relations, and the 'Reign of Law'", pages '55-190 in H. Frankfurt (ed.), Leibniz: A Collection of Critical Essays, Anchor Books, NY. Reprinted in pp. 259-286 of S. Knuuttila (ed.), Reforging the Great Chain of Being, Reidel, Dordrecht 1981. DOI: 10.1007/978-94015-7662-8_7

Hintikka, J., 1996, The Principles of Mathematics Revisited, Cambridge University Press, Cambridge. DOI: 10.1017/CB09780511624919

Hintikka, J., and L. Carlson, 1979, "Conditionals, generic quantifiers, and other applications of subgames", pages 179-214 in Saarinen [1979]. DOI: 10.1007/ 978-94-009-9775-2_1 
Hintikka, J., and J. Kulas, 1983, The Game of Language: Studies in GameTheoretical Semantics and its Applications, Reidel, Dordrecht. DOI: 10. 1007/978-1-4020-4108-2

Hintikka, J., and G. Sandu, 1989, "Informational independence as a semantical phenomenon", pages 571-589 in J.E. Fenstad, I. T. Frolov and R. Hilpinen (eds.), Logic, Methodology and Philosophy of Science, vol. 8, Elsevier, Amsterdam. (Reprinted as Chapter 3 in Hintikka, Paradigms for Language Theory and Other Essays, Kluwer, Dordrecht 1998.) DOI: 10.1016/S0049237X (08) 70066-1

Hodges, W., 1997, "Compositional semantics for a language of imperfect information", Logic Journal of the IGPL 5: 539-563. DOI: 10.1093/jigpal/5. 4.539

Hodges, W., 2007, "Logics of imperfect information: Why sets of assignments?", pages 117-133 in J. van Benthem, D. Gabbay and B. Löwe (eds.), Interactive Logic: Procs. 7th Augustus de Morgan Workshop, London, Amsterdam University Press, Amsterdam.

Hoeltje, M., B. Schnieder and A. Steinberg (eds.), 2013, Varieties of Dependence: Ontological Dependence, Grounding, Supervenience, ResponseDependence, Philosophia Verlag, Munich.

Holliday, W.H., 2014, "Partiality and adjointness in modal logic", pages 313332 in R. Goré, B. Kooi and A. Kurucz (eds.), Advances in Modal Logic, vol. 10, College Publications, London.

Holliday, W. H., 2016, "Possibility frames and forcing for modal logic", Working Papers of the Group in Logic and the Methodology of Science, UC Berkeley. http://escholarship.org/uc/item/9v11rodq

Horgan, T., 1993, "From supervenience to superdupervenience: Meeting the demands of a material world', Mind 102: 555-586. DOI: 10.1093/mind/ 102.408 .555

Horgan, T., and M. Timmons, 1992, "Troubles on moral twin earth: Moral queerness revived", Synthese 92: 221-260. DOI: 10.1007/BF00414300

Humberstone, L., 1974, "Topics in tense logic", B. Phil. Thesis, University of Oxford (supervisor: D. S. Scott).

Humberstone, L., 1979, "Interval semantics for tense logic: Some remarks", Journal of Philosophical Logic 8: 171-196. DOI: 10.1007/BF00258426

Humberstone, L., 1981, "From worlds to possibilities", Journal of Philosophical Logic 10: 313-339. DOI: 10.1007/BF00293423

Humberstone, L., 1982, "Scope and subjunctivity", Philosophia 12: 99-126. DOI: $10.1007 / \mathrm{BF} 02379362$ 
Humberstone, L., 1986, "Extensionality in sentence position", Journal of Philosophical Logic: 15: 27-54. See also "The Lattice of extensional connectives: A correction" Journal of Philosophical Logic 17 (1988): 221-223. DOI: 10. 1007/BF00250548 and for the correction DOI: 10.1007/BF00247953

Humberstone, L., 1987, "Critical Notice of [Hintikka and Kulas, 1983]", Mind 96: 99-107. DOI: 10.1093/mind/XCVI . 381.99

Humberstone, L., 1988a, "Operational semantics for positive R", Notre Dame Journal of Formal Logic 29: 61-80. DOI: 10.1305/ndjfl/1093637771

Humberstone, L., 1988b, "Heterogeneous logic", Erkenntnis 29: 395-435. DOI: $10.1007 / \mathrm{BF} 00183072$

Humberstone, L., 1991, "A study of some 'separated' conditions on binary relations", Theoria 57: 1-16. DOI: 10.1111/j.1755-2567.1991.tb00538.x Humberstone, L., 1992, "Some structural and logical aspects of the notion of supervenience", Logique et Analyse 35: 101-137.

Humberstone, L., 1993, "Functional dependencies, supervenience, and consequence relations", Journal of Logic, Language and Information 2: 309-336. DOI: $10.1007 / \mathrm{BF} 01181684$

Humberstone, L., 1995a, "The logic of non-contingency", Notre Dame Journal of Formal Logic 36: 214-229. DOI: 10.1305/ndjfl/1040248455

Humberstone, L., 1995b, "Comparatives and the reducibility of relations", Pacific Philosophical Quarterly 76: 117-141. DOI: 10.1111/j.1468-0114. 1995.tb00143.x

Humberstone, L., 1996a, "Classes of valuations closed under operations Galoisdual to Boolean sentence connectives", Publications of the Research Institute for Mathematical Sciences, Kyoto University 32: 9-84. DOI: 10.2977/ prims $/ 1195163180$

Humberstone, L., 1996b, "Intrinsic/extrinsic", Synthese 108: 205-267. DOI: $10.1007 / \mathrm{BF} 00413498$

Humberstone, L., 1996c, "A study in philosophical taxonomy", Philosophical Studies 83: 121-169. DOI: 10.1007/BF00354286

Humberstone, L., 1997, "Singulary extensional connectives: A closer look", Journal of Philosophical Logic 26: 341-356. DOI: 10.1023/A: 1004240612163

Humberstone, L., 1998a, "Note on supervenience and definability", Notre Dame Journal of Formal Logic 39: 243-252. DOI: 10.1305/ndjf1/1039293066

Humberstone, L., 1998b, "Yes, I agree'", Studies in Language 22: 619-659. DOI: $10.1075 / \mathrm{sl} .22 .3 .05 \mathrm{hum}$

Humberstone, L., 2000, "Parts and partitions", Theoria 66: 41-82. DOI: 10. 1111/j.1755-2567.2000.tb01144.x 
Humberstone, L., 2002, "The modal logic of agreement and noncontingency", Notre Dame Journal of Formal Logic 43: 95-127. DOI: 10.1305/ndjfl/ 1071509431

Humberstone, L., 2004a, "Two-dimensional adventures", Philosophical Studies 118: 17-65. DOI: 10.1023/B: PHIL.0000019542.43440.d1

Humberstone, L., 2004b, "Archetypal forms of inference", Synthese 141: 45-76. DOI: $10.1023 /$ B:SYNT . 0000035850.89516.e1

Humberstone, L., 2005a, "Modality", pages 534-614, Chapter 20, in F. C. Jackson and M. Smith (eds.), The Oxford Handbook of Contemporary Philosophy, Oxford University Press, Oxford and New York. DOI: 10.1093/oxfordhb/ 9780199234769.003 .0020

Humberstone, L., 2005b, "Logical discrimination", pages 207-228 in J.Y. Béziau (ed.), Logica Universalis: Towards a General Theory of Logic, Birkhäuser, Basel. DOI: 10.1007/978-3-7643-8354-1_12

Humberstone, L., 2011, The Connectives, MIT Press, Cambridge, MA.

Humberstone, L., 2013, "Aggregation and idempotence", Review of Symbolic Logic 6: 680-708. DOI: 10.1017/S175502031300021X

Humberstone, L., 2016, Philosophical Applications of Modal Logic, College Publications, London.

Jackson, F., 1998, From Metaphysics to Ethics: A Defence of Conceptual Analysis, Oxford University Press, Oxford. DOI: 10.1093/0198250614.001.0001

Janssen, T., 2002, "Independent choices and the interpretation of IF logic", Journal of Logic, Language and Information 11: 367-387. DOI: 10.1023/A: 1015542413718

Japaridze, G., 2006, "Propositional computability logic I", ACM Transactions on Computational Logic 7: 302-330. DOI: 10.1145/1131313.1131318

Japaridze, G, 2009, "In the beginning was game semantics", pages 249-350 in O. Majer, A.-V. Pietarinen, Ahti-Veikko and T. Tulenheimo (eds.), Games: Unifying Logic, Language, and Philosophy, Springer Dordrecht. DOI: 10. 1007/978-1-4020-9374-6_11

Karmo, T., 1988, "Some valid (but no sound) arguments trivially span the 'Is'-'Ought' Gap", Mind 97: 252-257. DOI: 10.1093/mind/XCVII.386. 252

Kenny, A., 1963, Action, Emotion and Will, Routledge and Kegan Paul, London.

Khamara, E. J., 1988, "Indiscernibles and the absolute theory of space and time", Studia Leibnitiana 20: 140-59.

Kim, J., 1978, "Supervenience and nomological incommensurables", American Philosophical Quarterly 15: 149-156.

Kim, J., 1984a, "Supervenience and supervenient causation", Southern Journal of Philosophy 22: 45-56; Supplement: 1983 Spindel Conference (ed. T. Horgan). DOI: $10.1111 / j .2041-6962.1984 . t b 01548 \cdot x$ 
Kim, J., 1984b, "Concepts of supervenience", Philosophy and Phenomenological Research 45: 153-176. (Reprinted as Chapter 4 in [Kim, 1993a] and as Chapter 3 in [Kim, 2002].) DOI: 10.2307/2107423

Kim, J., 1990, "Supervenience as a philosophical concept", Metaphilosophy 21: 1-27. (Reprinted as Chapter 8 in [Kim, 1993a].) DOI: 10.1111/j.14679973.1990.tb00830.x

Kim, J., 1993a, Supervenience and Mind: Selected Philosophical Essays, Cambridge University Press, Cambridge. DOI: 10.1017/CB09780511625220

Kim, J., 1993b, "Postscripts on supervenience", Chapter 9 in [Kim, 1993a] (also as Chapter 5 in [Kim, 2002]). DOI: 10.1017/CB09780511625220.010

Kim, J. (ed.), 2002, Supervenience, Ashgate Dartmouth, Aldershot.

Klagge, J. C., 1984, 'An alleged difficulty concerning moral properties", Mind 93: 370-380. DOI: 10.1093/mind/XCIII . 371.370

Klagge, J. C., 1987, "Supervenience: Perspectives v. possible worlds', Philosophical Quarterly 37: 312-315. DOI: 10.2307/2220401

Klagge, J.C., 1995, "Supervenience: Model theory or metaphysics?", pages 60-72 in [Savellos and Yalçin, 1995]. DOI: 10.1017/CB09780511663857.003

Komori, Y., 1986, "A new semantics for intuitionistic predicate logic", Studia Logica 45: 9-17. DOI: 10.1007/BF01881545

Kontinen, J., and J. Väänänen, 2011, "A remark on negation in dependence logic", Notre Dame Journal of Formal Logic 52: 55-65. DOI: 10.1215/ 00294527-2010-036

Kremer, M., 1988, "Kripke and the logic of truth", Journal of Philosophical Logic 17: 225-278. DOI: 10.1007/BF00247954

Kremer, P., 2016, "The revision theory of truth", in E. N. Zalta (ed.), Stanford Encyclopedia of Philosophy (Winter 2016 Edition), https://plato. stanford.edu/archives/win2016/entries/truth-revision/

Langton, R., and D. Lewis, 1998, "Defining 'Intrinsic'", Philosophy and Phenomenological Research 58: 333-345. DOI: 10.2307/2653512

Leitgeb, H., 2005 "What truth depends on", Journal of Philosophical Logic 34: 155-192. DOI: $10.1007 / \mathrm{s} 10992-004-3758-3$

Leuenberger, S., 2008, "Supervenience in metaphysics", Philosophy Compass 3: 749-762. DOI: $10.1111 / \mathrm{j} .1747-9991.2008 .00150 . x$

Leuenberger, S., 2009, "What is global supervenience?", Synthese 170: 115129. DOI: $10.1007 / \mathrm{s} 11229-008-9360-4$

Leuenberger, S., 2013, "Supervenience among classes of relations", 325-346 in [Hoeltje et al., 2013].

Lewis, D., 1973, Counterfactuals, Basil Blackwell, Oxford.

Lewis, D., 1974, "'Tensions", pages 49-61 in M.K. Munitz and P. K. Unger (eds.), Semantics and Philosophy, New York University Press, New York. DOI: $10.1093 / 0195032047.003 .0014$ 
Lewis, D., 1983a, "Extrinsic properties", Philosophical Studies 44: 197-200. DOI: $10.1007 / \mathrm{BF} 00354100$

Lewis, D., 1983b, "New work for a theory of universals", Australasian Journal of Philosophy 61: 343-377. DOI: 10.1080/00048408312341131

Lewis, D., 1986, On the Plurality of Worlds, Blackwell, Oxford.

Lewis, D., 1988, "Statements Partly About Observation", Philosophical Papers 17: 1-31. DOI: $10.1080 / 05568648809506282$

Lewis, D., 2001, "Redefining 'intrinsic'", Philosophy and Phenomenological Research 63: 381-398. DOI: 10.1111/j.1933-1592.2001.tb00111.x

Lewis, H A., 1985, "Is the mental supervenient on the physical?", pages 159172 in B. Vermazen and M. B. Hintikka (eds.), Essays on Davidson, Oxford University Press, Oxford.

McCawley, J. D., 1993, Everything that Linguists have Always Wanted to Know about Logic - But were ashamed to ask, University of Chicago Press, Chicago. (First Edn. 1981.)

McFetridge, I. G., 1985 "Supervenience, realism, necessity", Philosophical Quarterly 35: 245-258. DOI: 10.2307/2218904

McGinn, C., 1980, "Philosophical materialism", Synthese 44: 173-206. DOI: 10.1007/BF00413406

McKinsey, J. C. C., 1945, 'On the syntactical construction of systems of modal logic', Journal of Symbolic Logic 10: 83-94. DOI: 10.2307/2267027

McLaughlin, B. P., 1995, "Varieties of supervenience", pages 16-59 in [Savellos and Yalçin, 1995]. (Reprinted as Chapter 8 in [Kim, 2002].) DOI: 10.1017/ CB09780511663857.002

McLaughlin, B., and K. Bennett, 2014, "Supervenience", The Stanford Encyclopedia of Philosophy (Spring 2014 Edition), E. N. Zalta (ed.), https:// plato.stanford.edu/archives/spr2014/entries/supervenience/

Michaelis, L. A., 1993, " 'Continuity' within three scalar models: The polysemy of adverbial still", Journal of Semantics 10: 193-237. DOI: 10.1093/jos/ 10.3 .193

Miller, R. B., 1990, "Supervenience is a two-way street", Journal of Philosophy 87: 695-701. DOI: $10.2307 / 2026976$

Montgomery, H., and R. Routley, 1966, "Contingency and non-contingency bases for normal modal logics", Logique et Analyse 9: 318-328.

Moser, P.K., and J.D. Trout, 1995, "Physicalism, supervenience, and dependence", pages 87-217 in [Savellos and Yalçin, 1995]. DOI: 10.1017/ CB09780511663857.011

Moyer, M., 2008, "Weak and global supervenience are strong", Philosophical Studies 138: 125-150. DOI: 10.1007/s11098-006-9002-y

Noonan, H.W., 1987, "Supervenience", Philosophical Quarterly 37: 78-85. DOI: $10.2307 / 2220062$ 
Oddie, G., 1987, "Truthlikeness and the convexity of propositions", pages 197217 in T. Kuipers (ed.), What is Closer-to-the-Truth?, Rodopi, Amsterdam: Rodopi.

Oddie, G., and P. Tichý, 1990, "Resplicing properties in the supervenience base", Philosophical Studies 58, 3: 259-269. DOI: 10.1007/BF00368286

Ewa Orłowska, E., 1985, "Semantics of nondeterministic possible worlds", Bulletin of the Polish Academy of Sciences - Mathematics 33: 453-458.

Patton, T., 1989, "On Humberstone's semantics for branching quantifiers", Mind 98: 429-433. DOI: 10.1093/mind/XCVIII.391.429

Paull, R. C., and T. R. Sider, 1992, "In defense of global supervenience", Philosophy and Phenomenological Research 52: 833-854. (Reprinted as Chapter 12 in [Kim, 2002].) DOI: 10.2307/2107913

Petrie, B., 1987 "Global supervenience and reduction", Philosophy and Phenomenonological Research 48: 119-130. DOI: 10.2307/2107710

Pigden, C. R., 2012, "Identifying goodness", Australasian Journal of Philosophy 90: 93-109. DOI: 10.1080/00048402.2011.560167

Połacik, T., and L. Humberstone, 2018, "Classically archetypal rules", to appear in Review of Symbolic Logic.

Post, J.F., 1984, "Comments on Teller", Southern Journal of Philosophy 22: 163-167; Supplement: 1983 Spindel Conference (ed. T. Horgan). DOI: 10. 1111/j.2041-6962.1984.tb01556.x

Post, J.F., 1995, "Global' supervenient determination: Too permissive?", pages 73-100 in [Savellos and Yalçin, 1995]. (Reprinted as Chapter 11 in [Kim, 2002].) DOI: 10.1017/CB09780511663857.004

Priest, G., 2006, Doubt Truth to be a Liar, Oxford University Press, Oxford. DOI: $10.1093 / 0199263280.001 .0001$

Punčochář, V., 2015, "Weak negation in inquisitive semantics", Journal of Logic, Language and Information 24: 323-355. DOI: 10.1007/s10849-0159219-2

Rabinowicz, W., 1979, Universalizability: A Study in Morals and Metaphysics, Reidel, Dordrecht. DOI: 10.1007/978-94-009-9484-3

Rauszer, C. M., 1985, "An equivalence between theory of functional dependencies and a fragment of intuitionistic logic", Bull. Polish Acad. Sci. Math. 33: 571-579.

Read, S., 1981 "Review of [Saarinen, 1979]", Philosophical Books 22: 40-42.

Roelofsen, F., 2013, "Algebraic foundations for the semantic treatment of inquisitive content", Synthese190: 79-102.DOI:10.1007/s11229-013-0282-4

Roelofsen, F., 2016, "Two alternatives for disjunction: an inquisitive reconciliation", to appear in E. Onea, K. von Heusinger, and M. Zimmermann (eds.), Questions in Discourse, Brill Publishers, Leiden. Online version 
Rotolo, A., 2017, "Logics for normative supervenience", pages 1-23 in B. Brożek, A. Rotolo and J. Stelmach (eds.), Supervenience and Normativity, Law and Philosophy, Springer, Dordrecht. DOI: 10.1007/978-3-319-61046-7_1

Saarinen, E. (ed.), 1979, Game Theoretical Semantics, Reidel, Dordrecht. DOI: 10.1007/978-1-4020-4108-2

Sagiv, Y., C. Delobel, D. S. Parker and R. Fagin, 1981, "An equivalence between relational database dependencies and a fragment of propositional logic", Journal of the Association for Computing Machinery 28: 435-453. (Correction ibid. 34 (1987): 1016-1018.) DOI: 10.1145/322261.322263 and for Correction: $10.1145 / 31846.31853$

Sahlqvist, H., 1975, "Completeness and correspondence in the first and second order semantics for modal logic", pages 110-143 in S. Kanger (ed.), Procs. of the Third Scandinavian Logic Symposium, Uppsala 1973, North-Holland, Amsterdam. DOI: 10.1016/S0049-237X (08) 70728-6

Sano, K., 2015, "Avoiding impossibility theorems in radical inquisitive semantics", pages 107-120 in S. Ju, H. Liu and H. Ono (eds.), Modality, Semantics and Interpretations, Springer-Verlag Berlin Heidelberg. DOI: 10.1007/9783-662-47197-5_6

Savellos, E. A., and Ü. Yalçin (eds.), 1995, Supervenience: New Essays, Cambridge University Press, Cambridge. DOI: 10.1017/CB09780511663857

Schmitt, J., and M. Schroeder, 2011, "Supervenience arguments under relaxed assumptions", Philosophical Studies 155: 133-160. DOI: 10.1007/s11098010-9569-1

Schnieder, B., 2008, "Truth-functionality", Review of Symbolic Logic 1: 64-72. DOI: $10.1017 / \mathrm{S} 1755020308080052$

Schroeder, M., 2014, Explaining the Reasons We Share: Explanation and Expression in Ethics, vol. 1, Oxford University Press, Oxford. DOI: 10.1093/ acprof : oso/9780198713807.001.0001

Shafer-Landau, R., 1994, "Supervenience and moral realism", Ratio (new series) 7: 145-152. DOI: 10.1111/j.1467-9329.1994.tb00060.x

Shagrir, O., 2013, "Concepts of supervenience revisited", Erkenntnis 78: 469485. DOI: $10.1007 / \mathrm{s} 10670-012-9410-7$

Simons, M., 2005, "Dividing things up: The semantics of or and the modal/or interaction", Natural Language Semantics 13: 271-316. DOI: 10.1007/ s11050-004-2900-7

Singh, R., 2008, "On the interpretation of disjunction: Asymmetric, incremental, and eager for inconsistency", Linguistics and Philosophy 31: 245-260. DOI: $10.1007 / \mathrm{s} 10988-008-9038-\mathrm{x}$

Smiley, T., 1962, "The independence of connectives", Journal of Symbolic Logic 27: 426-436. DOI: $10.2307 / 2964550$

Smiley, T., 1996, "Rejection", Analysis 56: 1-9. DOI:10.1093/analys/56.1.1 
Sobel, J.H., 2001, "Blackburn's problem: On its not insignificant residue", Philosophy and Phenomenological Research 62: 361-383. DOI: 10.1111/j. 1933-1592.2001.tb00060.x

Sonderholm, J., 2007, "A logical response to Blackburn's supervenience argument", Sats - Nordic Journal of Philosophy 8: 178-185.

Stalnaker, R.C., 2003, "Varieties of supervenience", pages 86-108 in R. Stalnaker, Ways a World Might Be: Metaphysical and Anti-Metaphysical Essays, Oxford University Press, Oxford. DOI: 10.1093/0199251487.001. 0001 (Expanded version of a paper first published in 1996. DOI: 10.2307/ 2216245)

Steinberg, A., 2013, "Supervenience: A survey", pages 123-166 in [Hoeltje et al., 2013].

Steinberg, A., 2014, "Defining global supervenience", Erkenntnis 79: 367-380. DOI: $10.1007 / \mathrm{s} 10670-013-9498-4$

Taylor, B., 1985, Modes of Occurrence: Verbs, Adverbs and Events, Basil Blackwell, Oxford.

Teller, P., 1984, "Comments on Kim's paper", Southern Journal of Philosophy 22: 57-61; Supplement: 1983 Spindel Conference (ed. T. Horgan). DOI: 10. 1111/j.2041-6962.1984.tb01549.x

Teller, P., 1985, "Is supervenience just disguised reduction?", Southern Journal of Philosophy 23: 93-99. DOI: 10.1111/j.2041-6962.1985.tb00379.x

Tennant, N., 1985, "Beth's theorem and reductionism", Pacific Philosophical Quarterly 66: 342-54. DOI: 10.1111/j.1468-0114.1985.tb00258.x

Tennant, N., 1998, "Games some people would have all of us play: Critical study of Hintikka [Hintikka, 1996]", Philosophia Mathematica 6: 90-115. DOI: $10.1093 / \mathrm{philmat} / 6.1 .90$

Thomason, R. H., and H. Leblanc, 1967, "All or none: A novel choice of primitives for elementary logic", Journal of Symbolic Logic 32: 345-351. DOI: 10. $2307 / 2270776$

Tulenheimo, T., 2016, "Independence friendly logic", Stanford Encyclopedia of Philosophy (Winter 2016 Edition), E. N. Zalta (ed.), https://plato. stanford.edu/archives/win2016/entries/logic-if/

Tulenheimo, T., and M. Sevenster, 2007, "Approaches to independence friendly modal logic", pages 247-280 in J. van Benthem, D. Gabbay, B. Löwe (eds.), Interactive Logic: Procs. 7th Augustus de Morgan Workshop, Amsterdam University Press, Amsterdam.

Väänänen, J., 2007a, Dependence Logic: A New Approach to Independence Friendly Logic, London Mathematical Society student texts \#70, Cambridge University Press, Cambridge. 
Väänänen, J., 2007b, "Team logic", pages 281-302 in J. van Benthem, D. Gabbay, and B. Löwe (eds.), Interactive Logic: Procs. 7th Augustus de Morgan Workshop, London, Amsterdam University Press, Amsterdam.

Väänänen, J., 2008, "Modal dependence logic", pages 237-254 in K. R. Apt and R. van Rooij (eds.), New Perspectives on Games and Interaction, Amsterdam University Press, Amsterdam. DOI: 10.5117/9789089640574

Väänänen, J., and W. Hodges, 2010, "Dependence of variables construed as an atomic formula", Annals of Pure and Applied Logic 161: 817-828. DOI: 10. 1016/j.apal.2009.06.009

van Benthem, J., 2001, "Correspondence theory", pages 325-408 in D. Gabbay and F. Guenthner (eds.), Handbook of Philosophical Logic, Second Edition, vol. 3, Kluwer, Dordrecht. DOI: 10.1007/978-94-017-0454-0_4

van Benthem, J., 2005, "Minimal predicates, fixed-points, and definability", Journal of Symbolic Logic 70: 696-712. DOI: 10.2178/jsl/1122038910

Van Cleve, J., 1990, "Supervenience and closure", Philosophical Studies 58: 225-238. (Reprinted as Chapter 14 in [Kim, 2002].) DOI: 10.1007/ $\mathrm{BF} 00368283$

Van Fraassen, B., 1969, "Facts and tautological entailments", Journal of Philosophy 66: 477-487. DOI: $10.2307 / 2024563$

Verkuyl, H. J., 1989, "Aspectual classes and aspectual composition", Linguistics and Philosophy 12: 39-94. DOI: 10.1007/BF00627398

Verkuyl, H.J., 2005, "Aspectual composition: Surveying the ingredients", pages 19-39 in H.J. Verkuyl, H. de Swart and A. van Hout (eds.), Perspectives on Aspect, Springer, Dordrecht. DOI:10.1007/1-4020-3232-3_2

Weatherson, B., 2001, "Intrinsic properties and combinatorial principles", Philosophy and Phenomenological Research 63: 365-380. DOI: 10.1111/j. 1933-1592.2001.tb00110.x

Brian Weatherson and Dan Marshall, 2014, "Intrinsic vs. extrinsic properties", Stanford Encyclopedia of Philosophy (Fall 2014 Edition), E.N. Zalta (ed.), https://plato.stanford.edu/archives/fall2014/ entries/intrinsic-extrinsic/

Wedgwood, R., 2000, "The price of non-reductive physicalism", Noûs 34: 400421. DOI: $10.1111 / 0029-4624.00217$

Wedgwood, R., 2007, The Nature of Normativity, Oxford University Press, Oxford. DOI: 10.1093/acprof : oso/9780199251315.001.0001

Wehmeier, K. F., 2004, "Wittgensteinian predicate logic", Notre Dame Journal of Formal Logic 45: 1-11. DOI: 10.1305/ndjfl/1094155275

Wehmeier, K. F., 2012, "How to live without identity - and why", Australasian Journal of Philosophy 90: 761-777. DOI: 10.1080/00048402.2011.627927 
Williamson, T., 2001, "Ethics, supervenience and Ramsey sentences", Philosophy and Phenomenological Research 62: 625-630. DOI: 10.1111/j.19331592.2001.tb00079.x

Wilson, A., 2018, "Classifying Dependencies", to appear in G. Darby, D. Glick and A. Marmodoro (eds.), The Foundation of Reality: Fundamentality, Space and Time, Oxford University Press, Oxford.

Wilson, J., 2014, "No work for a theory of grounding", Inquiry 57: 535-579. DOI: $10.1080 / 0020174 \mathrm{X} .2014 .907542$

Wiredu, J.E., 1979, "On the necessity of S4", Notre Dame Journal of Formal Logic 20: 689-694. DOI: 10.1305/ndjfl/1093882679

Wright, G., 1985, "Review of [Blackburn, 1984]", Mind 94: 310-319. DOI: 10. 1093/mind/XCIV. 374.310

Yablo, S., 1982, "Grounding, dependence, and paradox", Journal of Philosophical Logic 11: 117-137. DOI: 10.1007/BF00302341

Yablo, S., 2014, Aboutness, Princeton University Press, Princeton NJ. DOI: $10.1515 / 9781400845989$

Yang, F., 2014, "On extensions and variants of dependence logic - A study of intuitionistic connectives in the team semantics setting", PhD Thesis, University of Helsinki.

Yang, F., 2017, "Modal dependence logics: Axiomatizations and modeltheoretic properties", Logic Journal of the IGPL 25: 773-805. DOI: 10. 1093/jigpal/jzx023

Yoshimi, J., 2007, "Supervenience, determination, and dependence", Pacific Philosophical Quarterly 88: 114-133. DOI: 10.1111/j.1468-0114.2007. 00283. $\mathrm{x}$

Zangwill, N., 1998, "Moral supervenience", Midwest Studies in Philosophy 20: 240-262. (Reprinted as Chapter 20 in [Kim, 2002].) DOI: 10.1111/j.14754975.1995.tb00315.x

Zangwill, N., 2008, "Moral dependence", pages 109-127 in by R. Shafer-Landau (ed.), Oxford Studies in Metaethics, vol. 3, Oxford University Press, Oxford.

Zimmermann, T.E., 2000, "Free choice disjunction and epistemic possibility", Natural Language Semantics 8: 259-290. DOI: 10.1023/A:1011255819284

\author{
LLOYd Humberstone \\ Department of Philosophy \\ School of Philosophical, Historical and International Studies \\ Monash University \\ Clayton, Victoria 3800, Australia \\ lloyd.humberstone@monash.edu
}

